EDITORIAL BOARD

ANTONIO CARCATERRA

ERIC A. CARLEN

FRANCESCO DELL'ISOLA

RAFFAELE ESPOSITO

ALBERT FANNJIANG

Gilles A. FrancFort

Pierangelo Marcati

JEAN-JACQUES MARIGO

PETER A. MARKOWICH

MARTIN OSTOJA-STARZEWSKI

PIERRE SEPPECHER

DAVID J. STEIGMANN

PAUL STEINMANN

PierRe M. SuQueT

MANAGING EDITORS

MICOL AMAR

ANGELA MADEO

MARTIN OSTOJA-STARZEWSKI

\section{ADVISORY BOARD}

ADNAN AKAY

Holm AltenbaCH

MICOL AMAR

HARM ASKES

TEODOR ATANACKOVIĆ

VICTOR BERDICHEVSKY

GuY BouchitTÉ

ANDREA BRAIDES

ROBERTO CAMASSA

MAURO CARFORE

ERIC DARVE

FELIX DARVE

ANNA DE MASI

Gianpietro Del Piero

Emmanuele Di Benedetto

VICTOR A. EREMEYEV

BERNOLD FIEDLER

IRENE M. GAMBA

DAVID Y. GAO

SERGEy GaVRILYUK

Timothy J. HeAley

DOMINIQUE JEULIN

ROgER E. KHAYAT

CORRADO LATTANZIO

ROBERT P. LIPTON

ANGELO LUONGO

ANGEla MADEO

JUAN J. MANFREDI

CARlo MARCHIORO

ANIL MISRA

ROBERTO NATALINI

PATRIZIO NEFF

Thomas J. Pence

ANDREY PIATNITSKI

ERRICO PRESUTtI

MARIO PUlVIRENTI

Lucio Russo

Miguel A. F. SANJUAN

PATRICK SElvaduraI

MIROSLAV ŠILHAVÝ

GUIDO SWEERS

ANTOINETTE TORDESILLAS

LEV TRUSKINOVSKY

JUAN J. L. VELÁZQUEZ

VINCENZO VESPRI

ANGELO VULPIANI msp.org/memocs

Università di Roma "La Sapienza", Italia

Rutgers University, USA

(CO-CHAIR) Università di Roma "La Sapienza", Italia

(TREASURER) Università dell'Aquila, Italia

University of California at Davis, USA

(CO-CHAIR) Université Paris-Nord, France

Università dell' Aquila, Italy

École Polytechnique, France

DAMTP Cambridge, UK, and University of Vienna, Austria

(CHAIR MANAGING EDITOR) Univ. of Illinois at Urbana-Champaign, USA

Université du Sud Toulon-Var, France

University of California at Berkeley, USA

Universität Erlangen-Nürnberg, Germany

LMA CNRS Marseille, France

Università di Roma "La Sapienza", Italia

Université de Lyon-INSA (Institut National des Sciences Appliquées), France (CHAIR MANAGING EDITOR) Univ. of Illinois at Urbana-Champaign, USA

Carnegie Mellon University, USA, and Bilkent University, Turkey

Otto-von-Guericke-Universität Magdeburg, Germany

Università di Roma "La Sapienza”, Italia

University of Sheffield, UK

University of Novi Sad, Serbia

Wayne State University, USA

Université du Sud Toulon-Var, France

Università di Roma Tor Vergata, Italia

University of North Carolina at Chapel Hill, USA

Università di Pavia, Italia

Stanford University, USA

Institut Polytechnique de Grenoble, France

Università dell' Aquila, Italia

Università di Ferrara and International Research Center MEMOCS, Italia

Vanderbilt University, USA

Gdansk University of Technology, Poland

Freie Universität Berlin, Germany

University of Texas at Austin, USA

Federation University and Australian National University, Australia

Université Aix-Marseille, France

Cornell University, USA

École des Mines, France

University of Western Ontario, Canada

Università dell' Aquila, Italy

Louisiana State University, USA

Università dell'Aquila, Italia

Université de Lyon-INSA (Institut National des Sciences Appliquées), France University of Pittsburgh, USA

Università di Roma "La Sapienza", Italia

University of Kansas, USA

Istituto per le Applicazioni del Calcolo "M. Picone", Italy

Universität Duisburg-Essen, Germany

Michigan State University, USA

Narvik University College, Norway, Russia

Università di Roma Tor Vergata, Italy

Università di Roma “La Sapienza”, Italia

Università di Roma “Tor Vergata", Italia

Universidad Rey Juan Carlos, Madrid, Spain

McGill University, Canada

Academy of Sciences of the Czech Republic

Universität zu Köln, Germany

University of Melbourne, Australia

École Polytechnique, France

Bonn University, Germany

Università di Firenze, Italia

Università di Roma La Sapienza, Italia

MEMOCS (ISSN 2325-3444 electronic, 2326-7186 printed) is a journal of the International Research Center for the Mathematics and Mechanics of Complex Systems at the Università dell'Aquila, Italy.

Cover image: "Tangle” by $\odot$ John Horigan; produced using the Context Free program (contextfreeart.org).

PUBLISHED BY

7 mathematical sciences publishers

nonprofit scientific publishing

http://msp.org/

(C) 2019 Mathematical Sciences Publishers 


\title{
EFFECTIVE COMPUTATION OF SO(3) AND O(3) LINEAR REPRESENTATION SYMMETRY CLASSES
}

\author{
MARC OLIVE
}

\begin{abstract}
We propose a general algorithm to compute all the symmetry classes of any $\mathrm{SO}(3)$ or $\mathrm{O}(3)$ linear representation. This method relies on a binary operator between sets of conjugacy classes of closed subgroups, called the clips. We compute explicit tables for this operation, which allows us to definitively solve the problem.
\end{abstract}

1. Introduction 203

2. A general theory of clips 205

3. Closed subgroups of $\mathrm{O}(3) \quad 207$

4. Symmetry classes for irreducible representations 209

5. Clips tables 211

$\begin{array}{lll}\text { Appendix A. } & \text { Proofs for } \mathrm{SO}(3) & 218\end{array}$

$\begin{array}{ll}\text { Appendix B. Proofs for } \mathrm{O}(3) & 227\end{array}$

References $\quad 235$

\section{Introduction}

The problem of finding the symmetry classes (also called isotropy classes) of a given Lie group linear representation is a difficult task in general, even for a compact group, where their number is known to be finite [Mostow 1957; Mann 1962].

It is only in 1996 that Forte and Vianello [1996] were able to clearly define the symmetry classes of tensor spaces. Such tensor spaces, with natural $\mathrm{O}(3)$ and $\mathrm{SO}(3)$ representations, appear in continuum mechanics via linear constitutive laws. Thanks to this clarification, Forte and Vianello obtained for the first time the eight symmetry classes of the $\mathrm{SO}(3)$ reducible representation on the space of elasticity tensors. One goal was to clarify and correct all the attempts already made to model the notion of symmetry in continuum mechanics, as initiated by Curie [1894], but strongly influenced by crystallography. The results were contradictory - some authors announced nine different elasticity anisotropies [Love 1944] while others

\section{Communicated by Francesco dell'Isola.}

MSC2010: 20C35, 22C05.

Keywords: isotropy classes, clips, linear representations, symmetry classes, tensorial representations. 
announced ten of them [Schouten 1951; Jagodzinski 1955; Fedorov 1968; Gurtin 1973]. Following Forte and Vianello, similar results were obtained in piezoelectricity [Weller 2004], photoelasticity [Forte and Vianello 1997], and flexoelasticity [Le Quang and He 2011].

Besides these results on symmetry classes in continuum mechanics, the subject has also been active in the mathematical community, especially due to its importance in bifurcation theory. For instance, Michel [1980] obtained the isotropy classes for irreducible $\mathrm{SO}(3)$ representations. These results were confirmed by Ihrig and Golubitsky [1984] and completed by the symmetry classes for $\mathrm{O}(3)$. Later, they were corrected by Chossat et al. [1990]. Thereafter, Chossat and Guyard [1994] calculated the symmetry classes of a direct sum of two irreducible representations of $\mathrm{SO}(3)$.

In this paper, we propose an algorithm - already mentioned in [Olive and Auffray 2013; 2014; Olive 2014] — to obtain the finite set of symmetry classes for any $\mathrm{O}(3)$ or $\mathrm{SO}(3)$ representation. Such an algorithm uses a binary operation defined over the set of conjugacy classes of a given group $G$ and that we decided to call the clips operation. This operation was almost formulated in [Chossat and Guyard 1994], but with no specific name, and only computed for SO(3) closed subgroups. As mentioned in [Chossat and Guyard 1996], the clips operation allows one to compute the set of symmetry classes $\mathfrak{I}(V)$ of a direct sum $V=V_{1} \oplus V_{2}$ of linear representations of a group $G$, if we know the symmetry classes for each individual representation $\mathfrak{I}\left(V_{1}\right)$ and $\mathfrak{I}\left(V_{2}\right)$.

We compute clips tables for all conjugacy classes of closed subgroups of $\mathrm{O}(3)$ and $\mathrm{SO}(3)$. The clips table for $\mathrm{SO}(3)$ was obtained in [Chossat and Guyard 1994], but we give here more detail on the proof and extend the calculation to conjugacy classes of closed $\mathrm{O}(3)$ subgroups. These results allow us to obtain, in a finite-step process, the set of symmetry classes for any reducible $\mathrm{O}(3)$ or $\mathrm{SO}(3)$ representation, so that for instance we directly obtain the sixteen symmetry classes of piezoelectric tensors.

The subject being particularly important for applications to tensorial properties of any order, the necessity of convincing correctness of the clips tables requires a complete and correct full article with sound proofs. The present article is therefore intended to be a final solution to the theoretical problem and provide the effective calculation of symmetry classes. Of course, we try to show the direct interest in the mechanics of continuous media (for this, references to [Olive and Auffray 2013; 2014] are important), but we have no other choice than to insist on the mathematical formulation of the problem.

The paper is organized as follow. In Section 2, which is close to [Chossat and Guyard 1996, §2.1], the theory of clips is introduced for a general group $G$ and applied in the context of symmetry classes where it is shown that isotropy classes 
of a direct sum correspond to the clips of their respective isotropy classes. In Section 3, we recall classical results on the classification of closed subgroups of $\mathrm{SO}(3)$ and $\mathrm{O}(3)$ up to conjugacy. Models for irreducible representations of $\mathrm{O}(3)$ and $\mathrm{SO}(3)$ and their symmetry classes are recalled in Section 4 . We then provide in the subsection beginning on page 214 some applications to tensorial mechanical properties, as the nonclassical example of Cosserat elasticity. The clips tables for $\mathrm{SO}(3)$ and $\mathrm{O}(3)$ are presented in Section 5. The details and proofs of how to obtain these tables are provided in Appendices A and B.

\section{A general theory of clips}

Given a group $G$ and a subgroup $H$ of $G$, the conjugacy class of $H$

$$
[H]:=\left\{g H g^{-1}: g \in G\right\}
$$

is a subset of $\mathscr{P}(G)$. We define $\operatorname{Conj}(G)$ to be the set of all conjugacy classes of a given group $G$ :

$$
\operatorname{Conj}(G):=\{[H]: H \subset G\}
$$

Recall that, on $\operatorname{Conj}(G)$, there is a preorder relation induced by inclusion. It is defined as

$$
\left[H_{1}\right] \preceq\left[H_{2}\right] \text { if } H_{1} \text { is conjugate to a subgroup of } H_{2} \text {. }
$$

When restricted to the closed subgroups of a topological compact group, this preorder relation becomes a partial order [Bredon 1972, Proposition 1.9] and defines the poset (partially ordered set) of conjugacy classes of closed subgroups of $G$.

We now define a binary operation called the clips operation on the $\operatorname{set} \operatorname{Conj}(G)$.

Definition 2.1. Given two conjugacy classes $\left[H_{1}\right]$ and $\left[H_{2}\right]$ of a group $G$, we define their clips as the subset of conjugacy classes

$$
\left[H_{1}\right] \odot\left[H_{2}\right]:=\left\{\left[H_{1} \cap g H_{2} g^{-1}\right]: g \in G\right\} .
$$

This definition immediately extends to two families (finite or infinite) $\mathscr{F}_{1}$ and $\mathscr{F}_{2}$ of conjugacy classes:

$$
\mathscr{F}_{1} \odot \mathscr{F}_{2}:=\bigcup_{\left[H_{i}\right] \in \mathscr{F}_{i}}\left[H_{1}\right] \odot\left[H_{2}\right] .
$$

Remark 2.2. The clips operation was already introduced, with no specific name, in [Chossat and Guyard 1994], the notation being $\boldsymbol{P}\left(H_{1}, H_{2}\right)$. In this article, the author only focus on the $\mathrm{SO}(3)$ case, with no meaning to deal with a general theory. 
This clips operation thus defined a binary operation on the set $\mathscr{P}(\operatorname{Conj}(G))$, which is associative and commutative. We have moreover

$$
[\mathbb{1}] \odot[H]=\{[\mathbb{1}]\} \quad \text { and } \quad[G] \odot[H]=\{[H]\} \text {, }
$$

for every conjugacy class $[H]$, where $\mathbb{1}:=\{e\}$ and $e$ is the identity element of $G$.

Consider now a linear representation $(V, \rho)$ of the group $G$. Given $v \in V$, its isotropy group (or symmetry group) is defined as

$$
G_{\boldsymbol{v}}:=\{g \in G: g \cdot \boldsymbol{v}=\boldsymbol{v}\}
$$

and its isotropy class is the conjugacy class $\left[G_{v}\right]$ of its isotropy group. The isotropy classes (or orbit types) of the representation $V$ is the family of all isotropy classes of vectors $v$ in $V$ :

$$
\mathfrak{I}(V):=\left\{\left[G_{v}\right]: v \in V\right\} .
$$

The central observation is that the isotropy classes of a direct sum of representations is obtained by the clips of their respective isotropy classes.

Lemma 2.3. Let $V_{1}$ and $V_{2}$ be two linear representations of $G$. Then

$$
\mathfrak{I}\left(V_{1} \oplus V_{2}\right)=\mathfrak{I}\left(V_{1}\right) \odot \mathfrak{I}\left(V_{2}\right) .
$$

Proof. Let $\left[G_{\boldsymbol{v}}\right]$ be some isotropy class for $\mathfrak{I}\left(V_{1} \oplus V_{2}\right)$ and write $\boldsymbol{v}=\boldsymbol{v}_{1}+\boldsymbol{v}_{2}$ where $\boldsymbol{v}_{i} \in V_{i}$. Note first that $G_{\boldsymbol{v}_{1}} \cap G_{\boldsymbol{v}_{2}} \subset G_{\boldsymbol{v}}$. Conversely given $g \in G_{\boldsymbol{v}}$, we get

$$
g \cdot \boldsymbol{v}=g \cdot \boldsymbol{v}_{1}+g \cdot \boldsymbol{v}_{2}=\boldsymbol{v}, \quad g \cdot \boldsymbol{v}_{i} \in V_{i},
$$

and thus $g \cdot \boldsymbol{v}_{i}=\boldsymbol{v}_{i}$. This shows that $G_{\boldsymbol{v}}=G_{\boldsymbol{v}_{1}} \cap G_{\boldsymbol{v}_{2}}$ and therefore that

$$
\mathfrak{I}\left(V_{1} \oplus V_{2}\right) \subset \mathfrak{I}\left(V_{1}\right) \odot \mathfrak{I}\left(V_{2}\right) .
$$

Conversely, let $[H]=\left[H_{1} \cap g H_{2} g^{-1}\right]$ in $\mathfrak{I}\left(V_{1}\right) \odot \Im\left(V_{2}\right)$ where $H_{i}=G_{\boldsymbol{v}_{i}}$ for some vectors $\boldsymbol{v}_{i} \in V_{i}$. Then, if we set

$$
\boldsymbol{v}=\boldsymbol{v}_{1}+g \cdot \boldsymbol{v}_{2},
$$

we have $G_{v}=H_{1} \cap g H_{2} g^{-1}$, as before, which shows that

$$
\left[H_{1} \cap g H_{2} g^{-1}\right] \in \mathfrak{I}\left(V_{1} \oplus V_{2}\right)
$$

and achieves the proof.

Using this lemma, we deduce a general algorithm to obtain the isotropy classes $\Im(V)$ of a finite-dimensional representation of a reductive algebraic group $G$, provided we know

(1) a decomposition $V=\bigoplus_{i} W_{i}$ into irreducible representations $W_{i}$,

(2) the isotropy classes $\mathfrak{I}\left(W_{i}\right)$ for the irreducible representations $W_{i}$, and 
(3) the tables of clips operations $\left[H_{1}\right] \odot\left[H_{2}\right]$ between conjugacy classes of closed subgroups $\left[H_{i}\right]$ of $G$.

In the sequel of this paper, we will successfully apply this program to the linear representations of $\mathrm{SO}(3)$ and $\mathrm{O}(3)$.

\section{Closed subgroups of $\mathrm{O}(3)$}

Every closed subgroup of SO(3) is conjugate to one of [Golubitsky et al. 1988] $\mathrm{SO}(3), \quad \mathrm{O}(2), \quad \mathrm{SO}(2), \quad \mathbb{D}_{n}(n \geq 2), \quad \mathbb{Z}_{n}(n \geq 2), \quad \mathbb{\mathbb { T }}, \quad \mathbb{O}, \quad \mathbb{}, \quad$ or $\quad \mathbb{1}$ where:

- $\mathrm{O}(2)$ is the subgroup generated by all the rotations around the $z$-axis and the order 2 rotation $r:(x, y, z) \mapsto(x,-y,-z)$ around the $x$-axis.

- $\mathrm{SO}(2)$ is the subgroup of all the rotations around the $z$-axis.

- $\mathbb{Z}_{n}$ is the unique cyclic subgroup of order $n$ of $\operatorname{SO}(2)\left(\mathbb{Z}_{1}=\{I\}\right)$.

- $\mathbb{D}_{n}$ is the dihedral group. It is generated by $\mathbb{Z}_{n}$ and $r:(x, y, z) \mapsto(x,-y,-z)$ $\left(\mathbb{D}_{1}=\{I\}\right)$.

- $\mathbb{T}$ is the tetrahedral group, the (orientation-preserving) symmetry group of the tetrahedron $\mathscr{T}_{0}$ defined in Figure 10. It has order 12.

- $\mathbb{O}$ is the octahedral group, the (orientation-preserving) symmetry group of the cube $\mathscr{C}_{0}$ defined in Figure 10. It has order 24.

- $\square$ is the icosahedral group, the (orientation-preserving) symmetry group of the dodecahedron $\mathscr{D}_{0}$ in Figure 11. It has order 60.

- $\mathbb{1}$ is the trivial subgroup, containing only the unit element.

The poset of conjugacy classes of closed subgroups of $\mathrm{SO}(3)$ is completely described by the inclusion of subgroups [Golubitsky et al. 1988]

$$
\begin{array}{ll}
\mathbb{Z}_{n} \subset \mathbb{D}_{n} \subset \mathrm{O}(2) & (n \geq 2), \\
\mathbb{Z}_{n} \subset \mathbb{Z}_{m} \text { and } \mathbb{D}_{n} \subset \mathbb{D}_{m} & (\text { if } n \text { divides } m), \\
\mathbb{Z}_{n} \subset \mathrm{SO}(2) \subset \mathrm{O}(2) & (n \geq 2),
\end{array}
$$

completed by $\left[\mathbb{Z}_{2}\right] \preceq\left[\mathbb{D}_{n}\right](n \geq 2)$ and by the arrows in Figure 1 (note that an arrow between the classes $\left[H_{1}\right]$ and $\left[H_{2}\right]$ means that $\left[H_{1}\right] \preceq\left[H_{2}\right]$ ), taking account of the exceptional subgroups $\mathbb{O}, \mathbb{T}$, and $\mathbb{\square}$.

Classification of $\mathrm{O}(3)$ closed subgroups is more involved [Ihrig and Golubitsky 1984; Sternberg 1994] and has been described using three types of subgroups. Given a closed subgroup $\Gamma$ of $\mathrm{O}(3)$ this classification runs as follows:

Type I: A subgroup $\Gamma$ is of type $\mathrm{I}$ if it is a subgroup of $\mathrm{SO}(3)$. 


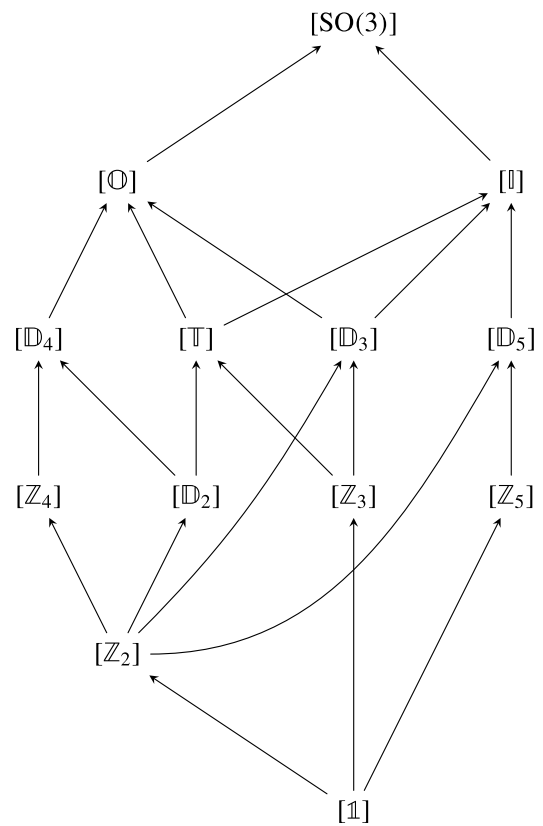

Figure 1. Exceptional conjugacy classes of closed $\mathrm{SO}(3)$ subgroups.

Type II: A subgroup $\Gamma$ is of type II if $-I \in \Gamma$. In that case, $\Gamma$ is generated by some subgroup $K$ of $\mathrm{SO}(3)$ and $-I$.

Type III: A subgroup $\Gamma$ is of type III if $-I \notin \Gamma$ and $\Gamma$ is not a subgroup of $\mathrm{SO}(3)$.

The description of type III subgroups requires more details. We will denote by $\boldsymbol{Q}(\boldsymbol{v} ; \theta) \in \mathrm{SO}(3)$ the rotation around $\boldsymbol{v} \in \mathbb{R}^{3}$ with angle $\theta \in[0,2 \pi[$ and by $\sigma_{v} \in \mathrm{O}(3)$ the reflection through the plane normal to $\boldsymbol{v}$. Finally, we fix an arbitrary orthonormal frame $(\boldsymbol{i}, \boldsymbol{j}, \boldsymbol{k})$, and we introduce the following definitions:

- $\mathbb{Z}_{2}^{-}$is the order-2 reflection group generated by $\sigma_{i}$ (where $\mathbb{Z}_{1}^{-}=\{\mathbb{1}\}$ ).

- $\mathbb{Z}_{2 n}^{-}(n \geq 2)$ is the group of order $2 n$, generated by $\mathbb{Z}_{n}$ and $-\boldsymbol{Q}(\boldsymbol{k} ; \pi / n)$ (see $(\mathrm{B}-1))$.

- $\mathbb{D}_{2 n}^{h}(n \geq 2)$ is the group of order $4 n$ generated by $\mathbb{D}_{n}$ and $-\boldsymbol{Q}(\boldsymbol{k} ; \pi / n)$ (see $(\mathrm{B}-3))$.

- $\mathbb{D}_{n}^{v}(n \geq 2)$ is the group of order $2 n$ generated by $\mathbb{Z}_{n}$ and $\sigma_{i}$ (where $\mathbb{D}_{1}^{v}=\{\mathbb{1}\}$ ).

- $\mathrm{O}(2)^{-}$is generated by $\mathrm{SO}(2)$ and $\sigma_{i}$.

These planar subgroups are completed by the subgroup $\mathbb{O}^{-}$which is of order 24 (see the subsection starting on page 233 and (B-5) for details).

The poset of conjugacy classes of closed subgroups of $\mathrm{O}(3)$ is given in Figure 2 for $\mathbb{O}^{-}$subgroups and in Figure 3 for $\mathrm{O}(3)$ subgroups. 


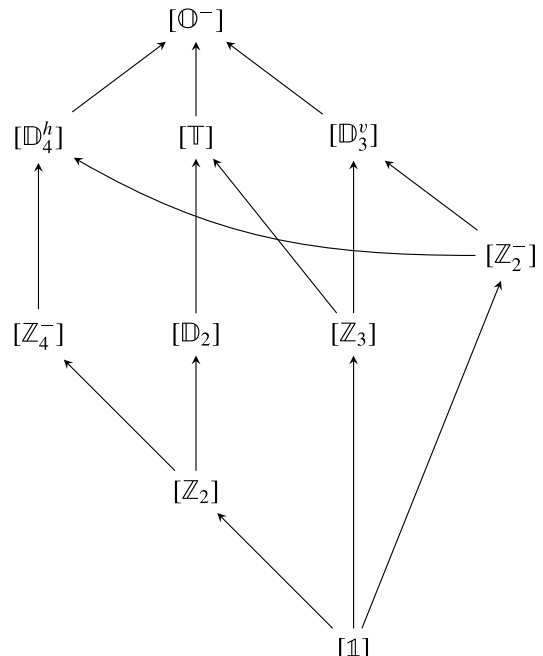

Figure 2. Poset of closed $\mathbb{O}^{-}$subgroups.

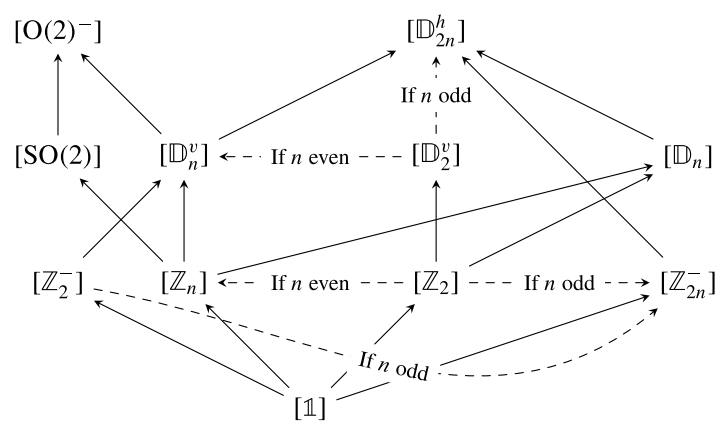

Figure 3. Poset of closed $\mathrm{O}(3)$ subgroups.

\section{Symmetry classes for irreducible representations}

Let $\mathscr{P}_{n}\left(\mathbb{R}^{3}\right)$ be the space of homogeneous polynomials of degree $n$ on $\mathbb{R}^{3}$. We have two natural representations of $\mathrm{O}(3)$ on $\mathscr{P}_{n}\left(\mathbb{R}^{3}\right)$. The first one, denoted by $\rho_{n}$, is given by

$$
\left[\rho_{n}(\mathrm{p})\right](\boldsymbol{x}):=\mathrm{p}\left(g^{-1} \boldsymbol{x}\right), \quad g \in G, \boldsymbol{x} \in \mathbb{R}^{3},
$$

whereas the second one, denoted by $\rho_{n}^{*}$, is given by

$$
\left[\rho_{n}^{*}(\mathrm{p})\right](\boldsymbol{x}):=\operatorname{det}(g) \mathrm{p}\left(g^{-1} \boldsymbol{x}\right), \quad g \in G, \boldsymbol{x} \in \mathbb{R}^{3} .
$$

Note that both of them induce the same representation $\rho_{n}$ of $\mathrm{SO}(3)$.

Let $\mathscr{H}_{n}\left(\mathbb{R}^{3}\right) \subset \mathscr{P}_{n}\left(\mathbb{R}^{3}\right)$ be the subspace of homogeneous harmonic polynomials of degree $n$ (polynomials with null Laplacian). It is a classical fact [Golubitsky 
et al. 1988] that $\left(\mathscr{H}_{n}\left(\mathbb{R}^{3}\right), \rho_{n}\right)$ and $\left(\mathscr{H}_{n}\left(\mathbb{R}^{3}\right), \rho_{n}^{*}\right)(n \geq 0)$ are irreducible $\mathrm{O}(3)$ representations, and each irreducible $\mathrm{O}(3)$ representation is isomorphic to one of them. Models for irreducible representations of $\mathrm{SO}(3)$ reduce to $\left(\mathscr{H}_{n}\left(\mathbb{R}^{3}\right), \rho_{n}\right)(n \geq 0)$.

Remark 4.1. Other classical models for $\mathrm{O}(3)$ and $\mathrm{SO}(3)$ irreducible representations, used in mechanics [Forte and Vianello 1996], are given by spaces of harmonic tensors (i.e., totally symmetric traceless tensors).

The isotropy classes for irreducible representations of $\mathrm{SO}(3)$ was first obtained by Michel [1980]. The same results were then obtained and completed in the $\mathrm{O}(3)$ case by Ihrig and Golubitsky [1984] and then by Chossat et al. [1990].

Theorem 4.2. The isotropy classes for the $\mathrm{SO}(3)$ representation $\left(\mathscr{H}_{n}\left(\mathbb{R}^{3}\right), \rho_{n}\right)$ are

(1) [1] for $n \geq 3$,

(2) $\left[\mathbb{Z}_{k}\right]$ for $2 \leq k \leq n$ if $n$ is odd and $2 \leq k \leq n / 2$ if $n$ is even,

(3) $\left[\mathbb{D}_{k}\right]$ for $2 \leq k \leq n$,

(4) [ए] for $n=3,6,7$ or $n \geq 9$,

(5) $[\mathbb{O}]$ for $n \neq 1,2,3,5,7,11$,

(6) [0] for $n=6,10,12,15,16,18$ or $n \geq 20$ and $n \neq 23,29$,

(7) $[\mathrm{SO}(2)]$ for $n$ odd,

(8) $[\mathrm{O}(2)]$ for $n$ even, and

(9) $[\mathrm{SO}(3)]$ for any $n$.

Remark 4.3. The list in Theorem 4.2 is similar to the list in [Michel 1980, Table A1; Chossat et al. 1990, Table A4]. In [Ihrig and Golubitsky 1984, Theorem 6.6] (for $\mathrm{SO}(3)$ irreducible representations)

- [ए] is an isotropy class for $n=6,7$ and $n \geq 9$ and

- $[\mathbb{O}]$ is an isotropy class for $n \neq 1,2,5,7,11$.

Such lists are different from (4) and (5) in our Theorem 4.2. But according to [Ihrig and Golubitsky 1984, Proposition 3.7], [『] is a maximum isotropy class for $n=3$. We have thus corrected this error in Theorem 4.2.

Theorem 4.4. The isotropy classes for the $\mathrm{O}(3)$ representations $\left(\mathscr{H}_{n}\left(\mathbb{R}^{3}\right), \rho_{n}\right)($ for $n$ odd) and $\left(\mathcal{H}_{n}\left(\mathbb{R}^{3}\right), \rho_{n}^{*}\right)$ (for $n$ even) are

(1) [1] for $n \geq 3$,

(2) $\left[\mathbb{Z}_{k}\right]$ for $2 \leq k \leq n / 2$,

(3) $\left[\mathbb{Z}_{2 k}^{-}\right]$for $k \leq n / 3$,

(4) $\left[\mathbb{D}_{k}\right]$ for $2 \leq k \leq n / 2$ if $n$ is odd and for $2 \leq k \leq n$ if $n$ is even,

(5) $\left[\mathbb{D}_{k}^{v}\right]$ for $2 \leq k \leq n$ if $n$ is odd and $2 \leq k \leq n / 2$ if $n$ is even, 
(6) $\left[\mathbb{D}_{2 k}^{h}\right]$ for $2 \leq k \leq n$, except $\mathbb{D}_{4}^{h}$ for $n=3$,

(7) [『] for $n \neq 1,2,3,5,7,8,11$,

(8) $[\mathbb{O}]$ for $n \neq 1,2,3,5,7,11$,

(9) $\left[\mathbb{O}^{-}\right]$for $n \neq 1,2,4,5,8$,

(10) [ [] for $n=6,10,12,15,16,18$ or $n \geq 20$ and $n \neq 23,29$,

(11) $[\mathrm{O}(2)]$ when $n$ is even, and

(12) $\left[\mathrm{O}(2)^{-}\right]$when $n$ is odd.

Remark 4.5. The list in Theorem 4.4 for $n$ odd is similar to the list in [Chossat et al. 1990, Table A4]. In [Ihrig and Golubitsky 1984, Theorem 6.8] (for O(3) irreducible representations), [ $\mathbb{\mathbb { T }}]$ is an isotropy class for $n \neq 1,2,5,7,8,11$ (which is different from the list (7) in our Theorem 4.4 above). But according to [Geymonat and Weller 2002; Weller 2004; Chossat et al. 1990, Table A4], [『] is not an isotropy class in the case $n=3$, and we corrected this error in the list (7) of Theorem 4.4.

\section{Clips tables}

SO(3) closed subgroups. The resulting conjugacy classes for the clips operation of closed $\mathrm{SO}(3)$ subgroups are given in Table 1.

The following notations have been used:

$$
\begin{aligned}
d & :=\operatorname{gcd}(m, n), & d_{2}:=\operatorname{gcd}(n, 2), & k_{2}:=3-d_{2}, \\
d_{3} & :=\operatorname{gcd}(n, 3), & d_{5}:=\operatorname{gcd}(n, 5), & \\
d z & :=\left\{\begin{array}{lll}
2 & \text { if } m \text { and } n \text { even, } & \text { otherwise, }
\end{array}\right. & d_{4}:= \begin{cases}4 & \text { if } 4 \text { divides } n, \\
1 & \text { otherwise, }\end{cases} & \mathbb{Z}_{1}=\mathbb{D}_{1}:=\mathbb{1} .
\end{aligned}
$$

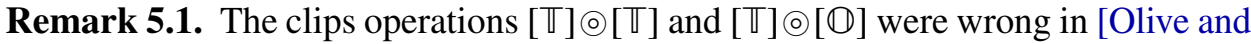
Auffray 2013; Olive 2014], since for instance the isotropy class $\left[\mathbb{D}_{2}\right]$ was omitted. Example 5.2 (isotropy classes for a family of $n$ vectors). For one vector, we get

$$
\mathfrak{I}\left(\mathscr{H}_{1}\left(\mathbb{R}^{3}\right)\right)=\{[\operatorname{SO}(2)],[\operatorname{SO}(3)]\} .
$$

From Table 1, we deduce that the isotropy classes for a family of $n$ vectors $(n \geq 2)$ is

$$
\mathfrak{I}\left(\bigoplus_{k=1}^{n} \mathscr{H}_{1}\left(\mathbb{R}^{3}\right)\right)=\{[\mathbb{1}],[\operatorname{SO}(2)],[\operatorname{SO}(3)]\} .
$$

Example 5.3 (isotropy classes for a family of $n$ quadratic forms). The space of quadratic forms on $\mathbb{R}^{3}, \mathrm{~S}_{2}\left(\mathbb{R}^{3}\right)$, decomposes into two irreducible components (deviatoric and spherical tensors for the mechanicians):

$$
\mathrm{S}_{2}\left(\mathbb{R}^{3}\right)=\mathscr{H}_{2}\left(\mathbb{R}^{3}\right) \oplus \mathscr{H}_{0}\left(\mathbb{R}^{3}\right) .
$$




\begin{tabular}{|c|c|c|c|c|c|c|c|}
\hline () & {$\left[\mathbb{Z}_{n}\right]$} & {$\left[\mathbb{D}_{n}\right]$} & [ए] & {$[\mathbb{O}]$} & [0] & {$[\mathrm{SO}(2)]$} & {$[\mathrm{O}(2)]$} \\
\hline$\left[\mathbb{Z}_{m}\right]$ & $\begin{array}{c}{[\mathbb{1}]} \\
{\left[\mathbb{Z}_{d}\right]}\end{array}$ & & & & & & \\
\hline$\left[\mathbb{D}_{m}\right]$ & $\begin{array}{c}{[\mathbb{1}]} \\
{\left[\mathbb{Z}_{d_{2}}\right]} \\
{\left[\mathbb{Z}_{d}\right]}\end{array}$ & $\begin{array}{c}{[\mathbb{1}],\left[\mathbb{Z}_{2}\right],\left[\mathbb{D}_{d z}\right]} \\
{\left[\mathbb{Z}_{d}\right],\left[\mathbb{D}_{d}\right]}\end{array}$ & & & & & \\
\hline [ए] & $\begin{array}{c}{[\mathbb{1}]} \\
{\left[\mathbb{Z}_{d_{2}}\right]} \\
{\left[\mathbb{Z}_{d_{3}}\right]}\end{array}$ & $\begin{array}{c}{[\mathbb{1}],\left[\mathbb{Z}_{2}\right]} \\
{\left[\mathbb{Z}_{d_{3}}\right],\left[\mathbb{D}_{d_{2}}\right]}\end{array}$ & $\begin{array}{c}{[\mathbb{1}],\left[\mathbb{Z}_{2}\right]} \\
{\left[\mathbb{D}_{2}\right],\left[\mathbb{Z}_{3}\right]} \\
{[\mathbb{\mathbb { T }}]}\end{array}$ & & & & \\
\hline$[\mathbb{O}]$ & $\begin{array}{c}{[\mathbb{1}]} \\
{\left[\mathbb{Z}_{d_{2}}\right]} \\
{\left[\mathbb{Z}_{d_{3}}\right]} \\
{\left[\mathbb{Z}_{d_{4}}\right]}\end{array}$ & $\begin{array}{c}{[\mathbb{1}],\left[\mathbb{Z}_{2}\right]} \\
{\left[\mathbb{Z}_{d_{3}}\right],\left[\mathbb{Z}_{d_{4}}\right]} \\
{\left[\mathbb{D}_{d_{2}}\right],\left[\mathbb{D}_{d_{3}}\right]} \\
{\left[\mathbb{D}_{d_{4}}\right]}\end{array}$ & $\begin{array}{c}{[\mathbb{1}]} \\
{\left[\mathbb{Z}_{2}\right],\left[\mathbb{D}_{2}\right]} \\
{\left[\mathbb{Z}_{3}\right]} \\
{[\mathbb{\mathbb { T }}]}\end{array}$ & $\begin{array}{c}{[\mathbb{1}],\left[\mathbb{Z}_{2}\right]} \\
{\left[\mathbb{D}_{2}\right],\left[\mathbb{Z}_{3}\right]} \\
{\left[\mathbb{D}_{3}\right],\left[\mathbb{Z}_{4}\right]} \\
{\left[\mathbb{D}_{4}\right],[\mathbb{O}]}\end{array}$ & & & \\
\hline [0] & $\begin{array}{c}{[\mathbb{1}]} \\
{\left[\mathbb{Z}_{d_{2}}\right]} \\
{\left[\mathbb{Z}_{d_{3}}\right]} \\
{\left[\mathbb{Z}_{d_{5}}\right]}\end{array}$ & $\begin{array}{c}{[\mathbb{1}],\left[\mathbb{Z}_{2}\right]} \\
{\left[\mathbb{Z}_{d_{3}}\right],\left[\mathbb{Z}_{d_{5}}\right]} \\
{\left[\mathbb{D}_{d_{2}}\right]} \\
{\left[\mathbb{D}_{d_{3}}\right],\left[\mathbb{D}_{d_{5}}\right]}\end{array}$ & $\begin{array}{l}{[\mathbb{1}]} \\
{\left[\mathbb{Z}_{2}\right]} \\
{\left[\mathbb{Z}_{3}\right]} \\
{[\mathbb{\mathbb { T }}]}\end{array}$ & $\begin{array}{c}{[\mathbb{1}],\left[\mathbb{Z}_{2}\right]} \\
{\left[\mathbb{Z}_{3}\right],\left[\mathbb{D}_{3}\right]} \\
{[\mathbb{\mathbb { T }}]}\end{array}$ & $\begin{array}{c}{[\mathbb{1}],\left[\mathbb{Z}_{2}\right]} \\
{\left[\mathbb{Z}_{3}\right],\left[\mathbb{D}_{3}\right]} \\
{\left[\mathbb{Z}_{5}\right],\left[\mathbb{D}_{5}\right]} \\
{[0]}\end{array}$ & & \\
\hline$[\mathrm{SO}(2)]$ & $\begin{array}{l}{[\mathbb{1}]} \\
{\left[\mathbb{Z}_{n}\right]}\end{array}$ & $\begin{array}{c}{[\mathbb{1}],\left[\mathbb{Z}_{2}\right]} \\
{\left[\mathbb{Z}_{n}\right]}\end{array}$ & $\begin{array}{c}{[\mathbb{1}],\left[\mathbb{Z}_{2}\right]} \\
{\left[\mathbb{Z}_{3}\right]}\end{array}$ & $\begin{array}{c}{[\mathbb{1}],\left[\mathbb{Z}_{2}\right]} \\
{\left[\mathbb{Z}_{3}\right],\left[\mathbb{Z}_{4}\right]}\end{array}$ & $\begin{array}{c}{[\mathbb{1}],\left[\mathbb{Z}_{2}\right]} \\
{\left[\mathbb{Z}_{3}\right],\left[\mathbb{Z}_{5}\right]}\end{array}$ & $\begin{array}{c}{[\mathbb{1}]} \\
{[\mathrm{SO}(2)]}\end{array}$ & \\
\hline$[\mathrm{O}(2)]$ & $\begin{array}{c}{[\mathbb{1}]} \\
{\left[\mathbb{Z}_{d_{2}}\right]} \\
{\left[\mathbb{Z}_{n}\right]}\end{array}$ & $\begin{array}{c}{[\mathbb{1}],\left[\mathbb{Z}_{2}\right]} \\
{\left[\mathbb{D}_{k_{2}}\right],\left[\mathbb{D}_{n}\right]}\end{array}$ & $\begin{array}{c}{[\mathbb{1}],\left[\mathbb{Z}_{2}\right]} \\
{\left[\mathbb{D}_{2}\right],\left[\mathbb{Z}_{3}\right]}\end{array}$ & $\begin{array}{c}{[\mathbb{1}],\left[\mathbb{Z}_{2}\right]} \\
{\left[\mathbb{D}_{2}\right],\left[\mathbb{D}_{3}\right]} \\
{\left[\mathbb{D}_{4}\right]}\end{array}$ & $\begin{array}{c}{[\mathbb{1}],\left[\mathbb{Z}_{2}\right]} \\
{\left[\mathbb{D}_{2}\right],\left[\mathbb{D}_{3}\right]} \\
{\left[\mathbb{D}_{5}\right]}\end{array}$ & $\begin{array}{c}{[\mathbb{1}]} \\
{\left[\mathbb{Z}_{2}\right]} \\
{[\operatorname{SO}(2)]}\end{array}$ & $\begin{array}{c}{\left[\mathbb{Z}_{2}\right]} \\
{\left[\mathbb{D}_{2}\right]} \\
{[\mathrm{O}(2)]}\end{array}$ \\
\hline
\end{tabular}

Table 1. Clips operations for $\mathrm{SO}(3)$.

We thus get

$$
\mathfrak{I}\left(\mathrm{S}_{2}\left(\mathbb{R}^{3}\right)\right)=\mathfrak{I}\left(\mathscr{H}_{2}\left(\mathbb{R}^{3}\right)\right)=\left\{\left[\mathbb{D}_{2}\right],[\mathrm{O}(2)],[\mathrm{SO}(3)]\right\} .
$$

The useful part of Table 1, for our purpose, reads:

\begin{tabular}{|c|ccc|}
\hline$\odot$ & {$\left[\mathbb{Z}_{2}\right]$} & {$\left[\mathbb{D}_{2}\right]$} & {$[\mathrm{O}(2)]$} \\
\hline$\left[\mathbb{Z}_{2}\right]$ & $\left\{[\mathbb{1}],\left[\mathbb{Z}_{2}\right]\right\}$ & $\left\{[\mathbb{1}],\left[\mathbb{Z}_{2}\right]\right\}$ & $\left\{[\mathbb{1}],\left[\mathbb{Z}_{2}\right]\right\}$ \\
{$\left[\mathbb{D}_{2}\right]$} & & $\left\{[\mathbb{1}],\left[\mathbb{Z}_{2}\right],\left[\mathbb{D}_{2}\right]\right\}$ & $\left\{[\mathbb{1}],\left[\mathbb{Z}_{2}\right],\left[\mathbb{D}_{2}\right]\right\}$ \\
{$[\mathrm{O}(2)]$} & & & $\left\{\left[\mathbb{Z}_{2}\right],\left[\mathbb{D}_{2}\right],[\mathrm{O}(2)]\right\}$ \\
\hline
\end{tabular}

We deduce therefore that the set of isotropy classes for a family of $n$ quadratic forms $(n \geq 2)$ is

$$
\Im\left(\bigoplus_{k=1}^{n} \mathrm{~S}_{2}\left(\mathbb{R}^{3}\right)\right)=\left\{[\mathbb{1}],\left[\mathbb{Z}_{2}\right],\left[\mathbb{D}_{2}\right],[\mathrm{O}(2)],[\mathrm{SO}(3)]\right\} .
$$




\begin{tabular}{|c|c|c|c|c|c|c|}
\hline ( ) & {$\left[\mathbb{Z}_{2}^{-}\right]$} & {$\left[\mathbb{Z}_{2 m}^{-}\right]$} & {$\left[\mathbb{D}_{m}^{v}\right]$} & {$\left[\mathbb{D}_{2 m}^{h}\right]$} & {$\left[\mathbb{O}^{-}\right]$} & {$\left[\mathrm{O}(2)^{-}\right]$} \\
\hline$\left[\mathbb{Z}_{2}^{-}\right]$ & {$[\mathbb{1}],\left[\mathbb{Z}_{2}^{-}\right]$} & & & & & \\
\hline$\left[\mathbb{Z}_{2 n}^{-}\right]$ & {$[\mathbb{1}],\left[\mathbb{Z}_{i(n)}^{-}\right]$} & Figure 4 , left & & & & \\
\hline$\left[\mathbb{D}_{n}^{v}\right]$ & {$[\mathbb{1}],\left[\mathbb{Z}_{2}^{-}\right]$} & Figure 4, right & $\begin{array}{c}{[\mathbb{1}],\left[\mathbb{Z}_{2}^{-}\right]} \\
{\left[\mathbb{D}_{d}^{v}\right],\left[\mathbb{Z}_{d}\right]}\end{array}$ & & & \\
\hline$\left[\mathbb{D}_{2 n}^{h}\right]$ & {$[\mathbb{1}],\left[\mathbb{Z}_{2}^{-}\right]$} & Figure 5 & Figure 6 & Figure 7 & & \\
\hline$\left[\mathbb{O}^{-}\right]$ & {$[\mathbb{1}],\left[\mathbb{Z}_{2}^{-}\right]$} & Figure 8 , left & $\begin{array}{c}{[\mathbb{1}],\left[\mathbb{Z}_{2}^{-}\right]} \\
{\left[\mathbb{Z}_{d_{3}(m)}\right]} \\
{\left[\mathbb{D}_{d_{3}(m)}^{v}\right]} \\
{\left[\mathbb{Z}_{d_{2}(m)}\right]} \\
{\left[\mathbb{D}_{d_{2}(m)}^{v}\right]}\end{array}$ & Figure 8 , right & $\begin{array}{c}{[\mathbb{1}],\left[\mathbb{Z}_{2}^{-}\right]} \\
{\left[\mathbb{Z}_{4}^{-}\right],\left[\mathbb{Z}_{3}\right]}\end{array}$ & \\
\hline$\left[\mathrm{O}(2)^{-}\right]$ & {$[\mathbb{1}],\left[\mathbb{Z}_{2}^{-}\right]$} & $\begin{array}{c}{[\mathbb{1}],\left[\mathbb{Z}_{i(m)}^{-}\right]} \\
{\left[\mathbb{Z}_{m}\right]}\end{array}$ & $\begin{array}{c}{[\mathbb{1}],\left[\mathbb{Z}_{2}^{-}\right]} \\
{\left[\mathbb{D}_{m}^{v}\right]}\end{array}$ & $\begin{array}{c}{[\mathbb{1}]} \\
{\left[\mathbb{Z}_{d_{2}(m)}\right],\left[\mathbb{Z}_{2}^{-}\right]} \\
{\left[\mathbb{D}_{i(m)}^{v}\right],\left[\mathbb{D}_{m}^{v}\right]}\end{array}$ & $\begin{array}{c}{[\mathbb{1}],\left[\mathbb{Z}_{2}^{-}\right]} \\
{\left[\mathbb{D}_{3}^{v}\right],\left[\mathbb{D}_{2}^{v}\right]}\end{array}$ & $\begin{array}{c}{\left[\mathbb{Z}_{2}^{-}\right]} \\
{\left[\mathrm{O}(2)^{-}\right]}\end{array}$ \\
\hline
\end{tabular}

Table 2. Clips operations on type III O(3) subgroups.

O(3) closed subgroups. Let us first consider an $\mathrm{O}(3)$ representation where $-I$ acts as - Id (meaning that this representation doesn't reduce to some $\mathrm{SO}(3)$ representation). In such a case, only the null vector can be fixed by -Id, and so type II subgroups never appear as isotropy subgroups. In that case, we only need to focus on clips operations between type I and type III subgroups, and then between type III subgroups, since clips operations between type I subgroups have already been considered in Table 1. For type III subgroups as detailed in Section B we have:

Lemma 5.4. Let $H_{1}$ be some type III closed subgroup of $\mathrm{O}(3)$ and $H_{2}$ be some type I closed subgroup of $\mathrm{O}(3)$. Then we have

$$
H_{1} \cap H_{2}=\left(H_{1} \cap \mathrm{SO}(3)\right) \cap H_{2},
$$

and for every closed subgroup $H$ of $\mathrm{SO}(3)$, we get

$$
\begin{aligned}
{\left[\mathbb{Z}_{2}^{-}\right] \odot[H] } & =\{[\mathbb{1}]\}, & {\left[\mathbb{Z}_{2 n}^{-}\right] \odot[H] } & =\left[\mathbb{Z}_{n}\right] \odot[H], \\
{\left[\mathbb{D}_{n}^{v}\right] \odot[H] } & =\left[\mathbb{Z}_{n}\right] \odot[H], & {\left[\mathbb{D}_{2 n}^{h}\right] \odot[H] } & =\left[\mathbb{D}_{n}\right] \odot[H], \\
{\left[\mathbb{O}^{-}\right] \odot[H] } & =[\mathbb{\mathbb { T }}] \odot[H], & {\left[\mathrm{O}(2)^{-}\right] \odot[H] } & =[\mathrm{SO}(2)] \odot[H] .
\end{aligned}
$$

The resulting conjugacy classes for the clips operation for type III subgroups are given in Table 2, where the following notations have been used:

$$
\begin{aligned}
d & :=\operatorname{gcd}(n, m), \quad d_{2}(n) & :=\operatorname{gcd}(n, 2), \\
d_{3}(n) & :=\operatorname{gcd}(n, 3), \quad i(n) & :=3-\operatorname{gcd}(2, n), \quad \mathbb{Z}_{1}^{-}=\mathbb{D}_{1}^{v}=\mathbb{D}_{2}^{h}=\mathbb{1} .
\end{aligned}
$$



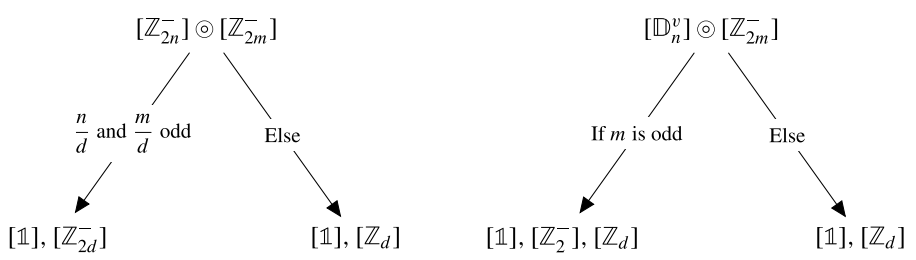

Figure 4. Left: $\mathbb{Z}_{2 n}^{-}$case. Right: $\mathbb{D}_{n}^{v}$ and $\mathbb{Z}_{2 n}^{-}$.

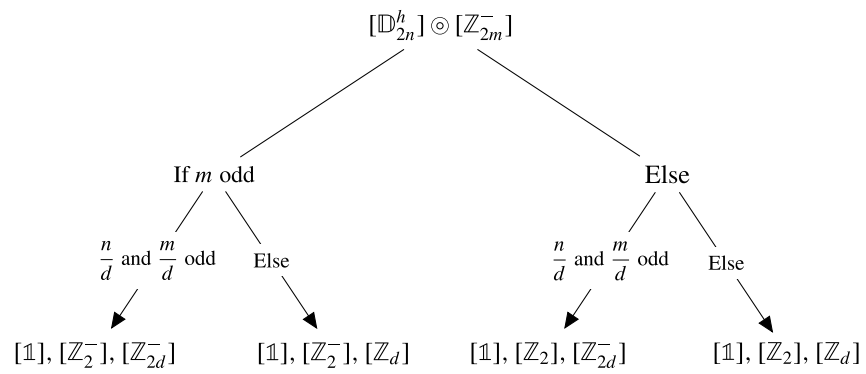

Figure 5. $\mathbb{D}_{2 n}^{h}$ and $\mathbb{Z}_{2 n}^{-}$.

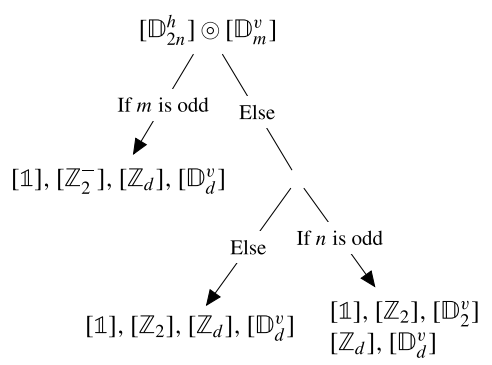

Figure 6. $\mathbb{D}_{2 n}^{h}$ and $\mathbb{D}_{n}^{v}$.

Remark 5.5. One misprint in [Olive 2014; Olive and Auffray 2014] for clips operation $\left[\mathrm{O}(2)^{-}\right] \odot\left[\mathbb{D}_{2 m}^{h}\right]$ has been corrected: the conjugacy class $\left[\mathbb{Z}_{2}\right]$ appears for $m$ even (and not for $m$ odd).

Application to tensorial mechanical properties. We propose here some direct applications of our results for many different tensorial spaces, each one being endowed with the natural $\mathrm{O}(3)$ representation. Such tensorial spaces occur for instance in the modeling of mechanical properties. The main idea is to use the irreducible decomposition, also known as the harmonic decomposition [Backus 1970; Forte and Vianello 1996]. We then use clips operations given in Tables 1 and 2. Note that we don't need to know explicit irreducible decomposition of those tensorial representations. 


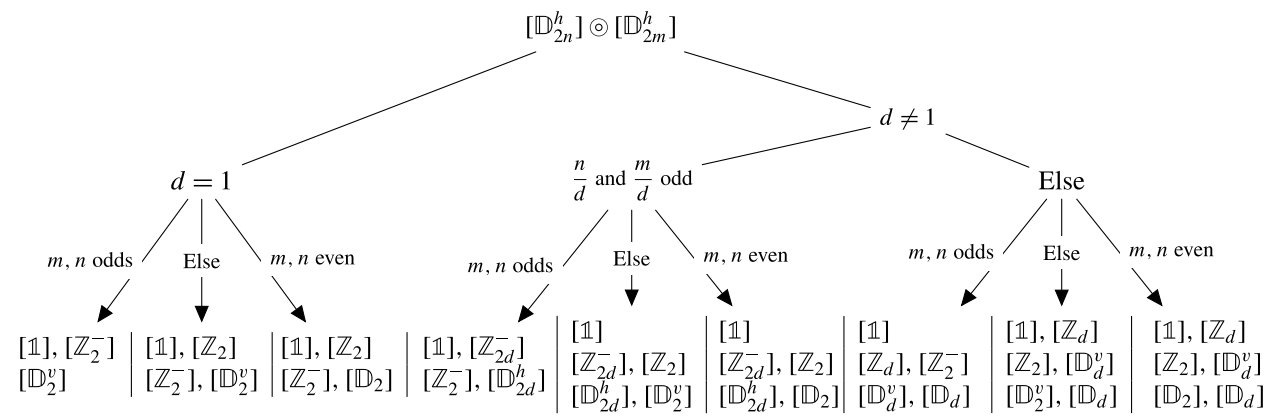

Figure 7. $\mathbb{D}_{2 n}^{h}$ and $\mathbb{D}_{2 m}^{h}$.

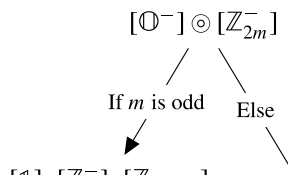

$[\mathbb{1}],\left[\mathbb{Z}_{2}^{-}\right],\left[\mathbb{Z}_{d_{3}(m)}\right]$

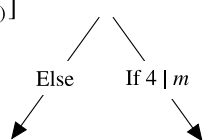

$[\mathbb{1}],\left[\mathbb{Z}_{4}^{-}\right],\left[\mathbb{Z}_{d_{3}(m)}\right] \quad[\mathbb{1}],\left[\mathbb{Z}_{2}\right],\left[\mathbb{Z}_{d_{3}(m)}\right]$

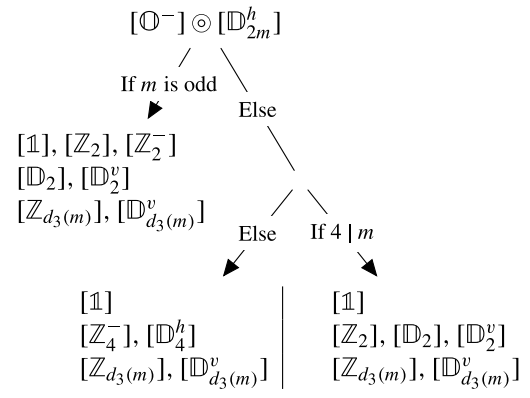

Figure 8. Left: $\mathrm{O}^{-}$and $\mathbb{Z}_{2 m}^{-}$. Right: $\mathrm{O}^{-}$and $\mathbb{D}_{2 m}^{h}$.

From now on, we define $\mathscr{H}_{n}\left(\mathbb{R}^{3}\right)^{*}$ to be the $\mathrm{O}(3)$ representation $\left(\mathscr{H}_{n}\left(\mathbb{R}^{3}\right), \rho_{n}^{*}\right)$. Furthermore, we define

$$
\mathscr{H}_{n}\left(\mathbb{R}^{3}\right)^{\oplus k}:=\bigoplus_{i=1}^{k} \mathcal{H}_{n}\left(\mathbb{R}^{3}\right), \quad \mathscr{H}_{n}\left(\mathbb{R}^{3}\right)^{\oplus k *}:=\bigoplus_{i=1}^{k} \mathcal{H}_{n}\left(\mathbb{R}^{3}\right)^{*} .
$$

We propose here some specific irreducible decompositions, related to mechanical theory, without any further details. We explain the use of the clips operation in the classical case of elasticity, all other examples being done in the same way.

Classical results. We first give some classical results we obtain here directly.

(1) Elasticity [Forte and Vianello 1996]: SO(3) tensor space

$$
\mathbb{E l a} \simeq \mathscr{H}_{4}\left(\mathbb{R}^{3}\right) \oplus \mathscr{H}_{2}\left(\mathbb{R}^{3}\right)^{\oplus 2} \oplus \mathscr{H}_{0}\left(\mathbb{R}^{3}\right)^{\oplus 2} .
$$

We have from Theorem 4.2

$$
\begin{aligned}
& \mathfrak{I}\left(\mathscr{H}_{2}\left(\mathbb{R}^{3}\right)\right)=\left\{\left[\mathbb{D}_{2}\right],[\mathrm{O}(2)],[\mathrm{SO}(3)]\right\}, \\
& \mathfrak{I}\left(\mathscr{H}_{4}\left(\mathbb{R}^{3}\right)\right)=\left\{[\mathbb{1}],\left[\mathbb{Z}_{2}\right],\left[\mathbb{D}_{2}\right],\left[\mathbb{D}_{3}\right],\left[\mathbb{D}_{4}\right],[\mathbb{O}],[\mathrm{O}(2)],[\operatorname{SO}(3)]\right\},
\end{aligned}
$$


so to obtain $\mathfrak{I}\left(\mathscr{H}_{2}\left(\mathbb{R}^{3}\right)^{\oplus 2}\right)$ we use the clips table

\begin{tabular}{|c|cc|}
\hline$\odot$ & {$\left[\mathbb{D}_{2}\right]$} & {$[\mathrm{O}(2)]$} \\
\hline$\left[\mathbb{D}_{2}\right]$ & $\left\{[\mathbb{1}],\left[\mathbb{Z}_{2}\right],\left[\mathbb{D}_{2}\right]\right\}$ & $\left\{[\mathbb{1}],\left[\mathbb{Z}_{2}\right],\left[\mathbb{D}_{2}\right]\right\}$ \\
{$[\mathrm{O}(2)]$} & & $\left\{\left[\mathbb{Z}_{2}\right],\left[\mathbb{D}_{2}\right],[\mathrm{O}(2)]\right\}$ \\
\hline
\end{tabular}

so that

$$
\begin{aligned}
\mathfrak{I}\left(\mathscr{H}_{2}\left(\mathbb{R}^{3}\right)^{\oplus 2}\right) & =\mathfrak{I}\left(\mathcal{H}_{2}\left(\mathbb{R}^{3}\right)\right) \odot \Im\left(\mathscr{H}_{2}\left(\mathbb{R}^{3}\right)\right) \\
& =\left(\left[\mathbb{D}_{2}\right] \odot\left[\mathbb{D}_{2}\right]\right) \cup\left(\left[\mathbb{D}_{2}\right] \odot\left[\mathrm{O}_{2}\right]\right) \cup\left(\left[\mathrm{O}_{2}\right] \odot\left[\mathrm{O}_{2}\right]\right) \\
& =\left\{[\mathbb{1}],\left[\mathbb{Z}_{2}\right],\left[\mathbb{D}_{2}\right],[\mathrm{O}(2)]\right\}
\end{aligned}
$$

and now we conclude using the clips table coming from the clips operation

$$
\mathfrak{I}(\mathbb{E l a})=\mathfrak{I}\left(\mathscr{H}_{4}\left(\mathbb{R}^{3}\right)\right) \odot \mathfrak{I}\left(\mathscr{H}_{2}\left(\mathbb{R}^{3}\right)^{\oplus 2}\right) .
$$

Finally we obtain eight symmetry classes

$$
\Im(\mathbb{E l a})=\left\{[\mathbb{1}],\left[\mathbb{Z}_{2}\right],\left[\mathbb{D}_{2}\right],\left[\mathbb{D}_{3}\right],\left[\mathbb{D}_{4}\right],[\mathbb{O}],[\mathrm{O}(2)],[\mathrm{SO}(3)]\right\} .
$$

(2) Photoelasticity [Forte and Vianello 1997]: SO(3) tensor space

$$
\mathbb{P l a} \simeq \mathscr{H}_{4}\left(\mathbb{R}^{3}\right) \oplus \mathscr{H}_{3}\left(\mathbb{R}^{3}\right) \oplus \mathscr{H}_{2}\left(\mathbb{R}^{3}\right)^{\oplus 3} \oplus \mathscr{H}_{1}\left(\mathbb{R}^{3}\right) \oplus \mathscr{H}_{0}\left(\mathbb{R}^{3}\right)^{\oplus 2}
$$

and twelve symmetry classes

$\mathfrak{I}(\mathbb{P l a})=\left\{[\mathbb{1}],\left[\mathbb{Z}_{2}\right],\left[\mathbb{D}_{2}\right],\left[\mathbb{Z}_{3}\right],\left[\mathbb{D}_{3}\right],\left[\mathbb{Z}_{4}\right],\left[\mathbb{D}_{4}\right],[\mathbb{T}],[\mathbb{O}],[\mathrm{SO}(2)],[\mathrm{O}(2)],[\mathrm{SO}(3)]\right\}$

(3) Piezoelectricity [Geymonat and Weller 2002]: O(3) tensor space

$$
\mathbb{P i e z} \simeq \mathscr{H}_{3}\left(\mathbb{R}^{3}\right) \oplus \mathscr{H}_{2}\left(\mathbb{R}^{3}\right)^{*} \oplus \mathscr{H}_{1}\left(\mathbb{R}^{3}\right)^{\oplus 2}
$$

and sixteen symmetry classes

$\Im(\mathbb{P i e z})=\left\{[\mathbb{1}],\left[\mathbb{Z}_{2}\right],\left[\mathbb{Z}_{3}\right],\left[\mathbb{D}_{2}^{v}\right],\left[\mathbb{D}_{3}^{v}\right],\left[\mathbb{Z}_{2}^{-}\right],\left[\mathbb{Z}_{4}^{-}\right],\left[\mathbb{D}_{2}\right],\left[\mathbb{D}_{3}\right],\left[\mathbb{D}_{4}^{h}\right],\left[\mathbb{D}_{6}^{h}\right]\right.$,

$$
\left.[\mathrm{SO}(2)],[\mathrm{O}(2)],\left[\mathrm{O}(2)^{-}\right],\left[\mathbb{O}^{-}\right],[\mathrm{O}(3)]\right\} \text {. }
$$

Remark 5.6. Note that the symmetry classes of the space (Piez, $\mathrm{O}(3)$ ) appear in many different works: in the PhD thesis of Weller [2004, Theorem 3.19, p. 84], where fourteen symmetry classes are announced, but fifteen appeared in the poset, in [Geymonat and Weller 2002] where they establish fourteen symmetry classes, in [Olive and Auffray 2014] (with a typo), in [Zou et al. 2013] (without the [O(3)] symmetry class), and finally in [Olive 2014], with sixteen symmetry classes. 
Nonclassical results. We now present some nonclassical tensor spaces, coming from Cosserat elasticity [Cosserat and Cosserat 1909; Eringen 1966; Forest 2005; Forest and Sievert 2006] and Strain gradient elasticity [Mindlin 1964; Mindlin and Eshel 1968; Portigal and Burstein 1968; Auffray et al. 2015], with their harmonic decomposition and symmetry classes.

(1) Classical Cosserat elasticity: $\mathrm{SO}(3)$ tensor space

$$
\mathbb{C} \text { os } \simeq \mathscr{H}_{4}\left(\mathbb{R}^{3}\right) \oplus \mathscr{H}_{3}\left(\mathbb{R}^{3}\right) \oplus \mathscr{H}_{2}\left(\mathbb{R}^{3}\right)^{\oplus 4} \oplus \mathscr{H}_{1}\left(\mathbb{R}^{3}\right)^{\oplus 2} \oplus \mathscr{H}_{0}\left(\mathbb{R}^{3}\right)^{\oplus 3}
$$

and twelve symmetry classes

$\Im(\mathbb{C o s})=\left\{[\mathbb{1}],\left[\mathbb{Z}_{2}\right],\left[\mathbb{Z}_{3}\right],\left[\mathbb{Z}_{4}\right],\left[\mathbb{D}_{2}\right],\left[\mathbb{D}_{3}\right],\left[\mathbb{D}_{4}\right],[\mathbb{\mathbb { T }}],[\mathbb{O}],[\mathrm{SO}(2)],[\mathrm{O}(2)],[\operatorname{SO}(3)]\right\}$

(2) Rotational Cosserat elasticity: $\mathrm{O}(3)$ tensor space

$$
\mathbb{C} h i \simeq \mathscr{H}_{4}\left(\mathbb{R}^{3}\right)^{*} \oplus \mathscr{H}_{3}\left(\mathbb{R}^{3}\right)^{\oplus 3} \oplus \mathscr{H}_{2}\left(\mathbb{R}^{3}\right)^{\oplus 6 *} \oplus \mathcal{H}_{1}\left(\mathbb{R}^{3}\right)^{\oplus 6} \oplus \mathcal{H}_{0}\left(\mathbb{R}^{3}\right)^{\oplus 3 *}
$$

and twenty-four symmetry classes

$$
\begin{array}{r}
\Im(\mathbb{C h i})=\left\{[\mathbb{1}],\left[\mathbb{Z}_{2}\right],\left[\mathbb{Z}_{3}\right],\left[\mathbb{Z}_{4}\right],\left[\mathbb{Z}_{2}^{-}\right],\left[\mathbb{Z}_{4}^{-}\right],\left[\mathbb{Z}_{6}^{-}\right],\left[\mathbb{D}_{2}\right],\left[\mathbb{D}_{3}\right],\left[\mathbb{D}_{4}\right],\right. \\
{\left[\mathbb{D}_{2}^{v}\right],\left[\mathbb{D}_{3}^{v}\right],\left[\mathbb{D}_{4}^{v}\right],\left[\mathbb{D}_{4}^{h}\right],\left[\mathbb{D}_{6}^{h}\right],\left[\mathbb{D}_{8}^{h}\right],[\mathbb{\mathbb { T }}],[\mathbb{O}],\left[\mathbb{O}^{-}\right],[\operatorname{SO}(2)],} \\
\left.[\mathrm{O}(2)],\left[\mathrm{O}(2)^{-}\right],[\operatorname{SO}(3)],[\mathrm{O}(3)]\right\} .
\end{array}
$$

(3) Fifth-order $\mathrm{O}(3)$ tensor space in strain gradient elasticity, given by

$$
\text { Sge } \simeq \mathscr{H}_{5}\left(\mathbb{R}^{3}\right) \oplus \mathcal{H}_{4}\left(\mathbb{R}^{3}\right)^{\oplus 2 *} \oplus \mathscr{H}_{3}\left(\mathbb{R}^{3}\right)^{\oplus 5} \oplus \mathscr{H}_{2}\left(\mathbb{R}^{3}\right)^{\oplus 5 *} \oplus \mathscr{H}_{1}\left(\mathbb{R}^{3}\right)^{\oplus 6} \oplus \mathcal{H}_{0}\left(\mathbb{R}^{3}\right)^{*}
$$

and twenty-nine symmetry classes

$$
\begin{array}{r}
\Im(S g e)=\left\{[\mathbb{1}],\left[\mathbb{Z}_{2}\right],\left[\mathbb{Z}_{3}\right],\left[\mathbb{Z}_{4}\right],\left[\mathbb{Z}_{5}\right],\left[\mathbb{Z}_{2}^{-}\right],\left[\mathbb{Z}_{4}^{-}\right],\left[\mathbb{Z}_{6}^{-}\right],\left[\mathbb{Z}_{8}^{-}\right],\left[\mathbb{D}_{2}\right],\left[\mathbb{D}_{3}\right],\left[\mathbb{D}_{4}\right],\left[\mathbb{D}_{5}\right],\right. \\
{\left[\mathbb{D}_{2}^{v}\right],\left[\mathbb{D}_{3}^{v}\right],\left[\mathbb{D}_{4}^{v}\right],\left[\mathbb{D}_{5}^{v}\right],\left[\mathbb{D}_{4}^{h}\right],\left[\mathbb{D}_{6}^{h}\right],\left[\mathbb{D}_{8}^{h}\right],\left[\mathbb{D}_{10}^{h}\right],[\mathbb{\mathbb { T }}],[\mathbb{O}],\left[\mathbb{O}^{-}\right],} \\
\left.[\mathrm{SO}(2)],[\mathrm{O}(2)],\left[\mathrm{O}(2)^{-}\right],[\operatorname{SO}(3)],[\mathrm{O}(3)]\right\} .
\end{array}
$$

(4) Fifth-order $\mathrm{O}(3)$ tensor space of acoustical gyrotropic tensor [Portigal and Burstein 1968] (reducing to fourth-order tensor space), given by

$$
\text { Agy } \simeq \mathscr{H}_{4}\left(\mathbb{R}^{3}\right)^{*} \oplus \mathscr{H}_{3}\left(\mathbb{R}^{3}\right)^{\oplus 2} \oplus \mathscr{H}_{2}\left(\mathbb{R}^{3}\right)^{\oplus 3 *} \oplus \mathscr{H}_{1}\left(\mathbb{R}^{3}\right)^{\oplus 2} \oplus \mathscr{H}_{0}\left(\mathbb{R}^{3}\right)^{*}
$$

and twenty-four symmetry classes

$$
\begin{aligned}
& \Im(\mathbb{A g y})=\left\{[\mathbb{1}],\left[\mathbb{Z}_{2}\right],\left[\mathbb{Z}_{3}\right],\left[\mathbb{Z}_{4}\right],\left[\mathbb{Z}_{2}^{-}\right],\left[\mathbb{Z}_{4}^{-}\right],\left[\mathbb{Z}_{6}^{-}\right],\right. \\
& {\left[\mathbb{D}_{2}\right],\left[\mathbb{D}_{3}\right],\left[\mathbb{D}_{4}\right],\left[\mathbb{D}_{2}^{v}\right],\left[\mathbb{D}_{3}^{v}\right],\left[\mathbb{D}_{4}^{v}\right],\left[\mathbb{D}_{4}^{h}\right],\left[\mathbb{D}_{6}^{h}\right],\left[\mathbb{D}_{8}^{h}\right], } \\
& {\left.[\mathbb{\mathbb { T }}],[\mathbb{O}],\left[\mathbb{O}^{-}\right],[\operatorname{SO}(2)],[\mathrm{O}(2)],\left[\mathrm{O}(2)^{-}\right],[\operatorname{SO}(3)],[\mathrm{O}(3)]\right\} . }
\end{aligned}
$$




\section{Appendix A: Proofs for SO(3)}

In this section, we provide all the details required to obtain the results in Table 1 . We will start with the following definition, which was introduced in [Golubitsky et al. 1988] and happens to be quite useful for this task.

Definition A.1. Let $K_{1}, K_{2}, \ldots, K_{s}$ be subgroups of a given group $G$. We say that $G$ is the direct union of the $K_{i}$ and we write $G=\biguplus_{i=1}^{s} K_{i}$ if

$$
G=\bigcup_{i=1}^{s} K_{i} \quad \text { and } \quad K_{i} \cap K_{j}=\{e\} \quad \text { for all } i \neq j .
$$

In the following, we will have to repeatedly identify the conjugacy class of intersections such as

$$
H_{1} \cap\left(g H_{2} g^{-1}\right),
$$

where $H_{1}$ and $H_{2}$ are two closed subgroups of $\mathrm{SO}(3)$ and $g \in \mathrm{SO}(3)$. A useful observation is that all closed $\mathrm{SO}(3)$ subgroups have some characteristic axes and that intersection (A-1) depends only on the relative positions of these characteristic axes.

As detailed below, for any subgroup conjugate to $\mathbb{Z}_{n}$ or $\mathbb{D}_{n}(n \geq 3)$, the axis of an $n$-th order rotation (in this subgroup) is called its primary axis. For subgroups conjugate to $\mathbb{D}_{n}(n \geq 3)$, axes of order-2 rotations are said to be secondary axes. In the special case $n=2$, the $z$-axis is the primary axis of $\mathbb{Z}_{2}$, while any of the $x$-, $y$-, or $z$-axes can be considered as a primary axis of $\mathbb{D}_{2}$.

Cyclic subgroup. For any axis $a$ of $\mathbb{R}^{3}$ (throughout the origin), we denote by $\mathbb{Z}_{n}^{a}$ the unique cyclic subgroup of order $n$ around the $a$-axis, which is its primary axis. We have then:

Lemma A.2. Let $m, n \geq 2$ be two integers and $d=\operatorname{gcd}(n, m)$. Then

$$
\left[\mathbb{Z}_{n}\right] \odot\left[\mathbb{Z}_{m}\right]=\left\{[\mathbb{1}],\left[\mathbb{Z}_{d}\right]\right\} .
$$

Proof. We have to consider intersections, such as

$$
\mathbb{Z}_{n} \cap\left(g \mathbb{Z}_{m} g^{-1}\right)=\mathbb{Z}_{n} \cap \mathbb{Z}_{m}^{a},
$$

for some axis $a$, and only two cases occur:

(1) If $a \neq(O z)$, then necessarily the intersection reduces to $\mathbb{1}$.

(2) If $a=(O z)$, then the order $r$ of a rotation in $\mathbb{Z}_{n} \cap \mathbb{Z}_{m}$ divides both $n$ and $m$ and thus divides $d=\operatorname{gcd}(m, n)$. We therefore get $\mathbb{Z}_{n} \cap \mathbb{Z}_{m} \subset \mathbb{Z}_{d}$. But obviously, $\mathbb{Z}_{d} \subset \mathbb{Z}_{n} \cap \mathbb{Z}_{m}$ and thus $\mathbb{Z}_{n} \cap \mathbb{Z}_{m}=\mathbb{Z}_{d}$. 


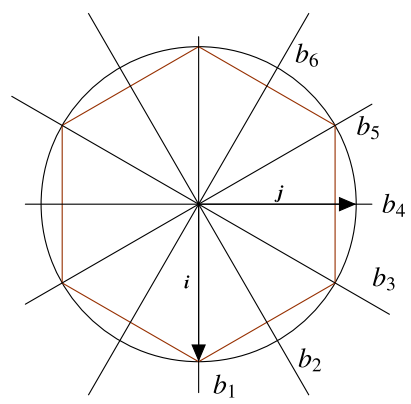

Figure 9. Secondary axis of the dihedral group $\mathbb{D}_{6}$.

Dihedral subgroup. Let $b_{1}$ be the $x$-axis and $b_{k}(k=2, \ldots, n)$ be the axis recursively defined by

$$
b_{k}:=Q\left(\boldsymbol{k} ; \frac{\pi}{n}\right) b_{k-1}
$$

Then, we have

$$
\mathbb{D}_{n}=\mathbb{Z}_{n} \uplus \mathbb{Z}_{2}^{b_{1}} \uplus \cdots \uplus \mathbb{Z}_{2}^{b_{n}},
$$

where the $z$-axis (corresponding to an $n$-th order rotation) is the primary axis and the $b_{k}$-axes (corresponding to order- 2 rotations) are the secondary axes of this dihedral group (see Figure 9).

Lemma A.3. Let $m, n \geq 2$ be two integers, and set $d:=\operatorname{gcd}(n, m)$ and $d_{2}(m):=$ $\operatorname{gcd}(m, 2)$. Then we have

$$
\left[\mathbb{D}_{n}\right] \odot\left[\mathbb{Z}_{m}\right]=\left\{[\mathbb{1}],\left[\mathbb{Z}_{d_{2}(m)}\right],\left[\mathbb{Z}_{d}\right]\right\} .
$$

Proof. Let $\Gamma=\mathbb{D}_{n} \cap g \mathbb{Z}_{m} g^{-1}$ for $g \in \mathrm{SO}$ (3). From decomposition (A-2), we have to consider intersections

$$
\mathbb{Z}_{n} \cap g \mathbb{Z}_{m} g^{-1}, \quad \mathbb{Z}_{2}^{b_{j}} \cap g \mathbb{Z}_{m} g^{-1} .
$$

which thus reduce to Lemma A.2.

Lemma A.4. Let $m, n \geq 2$ be two integers. Set $d:=\operatorname{gcd}(n, m)$ and

$$
d z:= \begin{cases}2 & \text { if } m \text { and } n \text { even } \\ 1 & \text { otherwise }\end{cases}
$$

Then, we have

$$
\left[\mathbb{D}_{n}\right] \odot\left[\mathbb{D}_{m}\right]=\left\{[\mathbb{1}],\left[\mathbb{Z}_{2}\right],\left[\mathbb{D}_{d z}\right],\left[\mathbb{Z}_{d}\right],\left[\mathbb{D}_{d}\right]\right\} .
$$

Proof. Let $\Gamma=\mathbb{D}_{n} \cap\left(g \mathbb{D}_{m} g^{-1}\right)$.

(1) If both primary axes and one secondary axis match, $\Gamma=\mathbb{D}_{d}$ if $d \neq 1$ and $\Gamma \in\left[\mathbb{Z}_{2}\right]$ otherwise. 


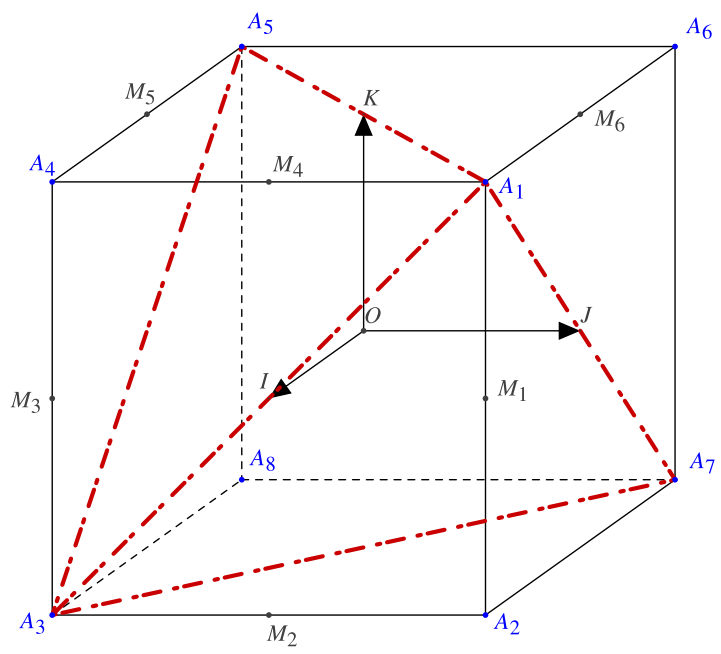

Figure 10. Cube $\mathscr{C}_{0}$ and tetrahedron $\mathscr{T}_{0}:=A_{1} A_{3} A_{7} A_{5}$.

(2) If only the primary axes match, $\Gamma=\mathbb{Z}_{d}$.

(3) If the angle of primary axes is $\pi / 4$ and a secondary axis match, then $\Gamma \in\left[\mathbb{Z}_{2}\right]$

(4) If the primary axis of $g \mathbb{D}_{m} g^{-1}$ matches with the secondary axis $(O x)$ of $\mathbb{D}_{n}$ (or the converse), we obtain $\Gamma \in\left[\mathbb{D}_{2}\right]$ for $n$ and $m$ even and a secondary axis of $g \mathbb{D}_{m} g^{-1}$ is $(O z)$; otherwise, we obtain $\Gamma \in\left[\mathbb{Z}_{2}\right]$.

(5) In all other cases, we have $\Gamma=\mathbb{1}$.

Tetrahedral subgroup. The (orientation-preserving) symmetry group $\mathbb{T}$ of the tetrahedron $\mathscr{T}_{0}:=A_{1} A_{3} A_{7} A_{5}$ (see Figure 10) decomposes as [Ihrig and Golubitsky 1984]

$$
\mathbb{\mathbb { V }}=\biguplus_{i=1}^{4} \mathbb{Z}_{3}^{v \boldsymbol{t}_{i}} \uplus \biguplus_{j=1}^{3} \mathbb{Z}_{2}^{\boldsymbol{e t} \boldsymbol{t}_{j}}
$$

where $\boldsymbol{v} t_{i}$ and $\boldsymbol{e t}{ }_{j}$ are the vertices axes and edges axes of the tetrahedron (see Figure 10), respectively:

$$
\begin{array}{llll}
v t_{1}:=\left(O A_{1}\right), & v t_{2}:=\left(O A_{3}\right), & \boldsymbol{v t}_{3}:=\left(O A_{5}\right), & \boldsymbol{v} \boldsymbol{t}_{4}:=\left(O A_{7}\right), \\
\boldsymbol{e t}_{1}:=(O x), & \text { et }_{2}:=(O y), & \boldsymbol{e t}_{3}:=(O z) . &
\end{array}
$$

Corollary A.5. Let $n \geq 2$ be an integer. Set $d_{2}(n):=\operatorname{gcd}(n, 2)$ and $d_{3}(n):=$ $\operatorname{gcd}(3, n)$. Then we have

$$
\left[\mathbb{Z}_{n}\right] \odot[\mathbb{\mathbb { V }}]=\left\{[\mathbb{1}],\left[\mathbb{Z}_{d_{2}(n)}\right],\left[\mathbb{Z}_{d_{3}(n)}\right]\right\}
$$


Proof. Consider $\mathbb{\mathbb { N }} \cap \mathbb{Z}_{n}^{a}$ for some axis $a$. As a consequence of Lemma A.2, we only need to consider the case where $a$ is an edge axis or a face axis of the tetrahedron, reducing to the clips operations

$$
\left[\mathbb{Z}_{2}\right] \odot\left[\mathbb{Z}_{n}\right], \quad\left[\mathbb{Z}_{3}\right] \odot\left[\mathbb{Z}_{n}\right],
$$

which directly leads to the lemma.

Corollary A.6. Let $n \geq 2$ be some integer. Set $d_{2}(n):=\operatorname{gcd}(n, 2)$ and $d_{3}(n):=$ $\operatorname{gcd}(3, n)$. Then we have

$$
\left[\mathbb{D}_{n}\right] \odot[\mathbb{\mathbb { V }}]=\left\{[\mathbb{1}],\left[\mathbb{Z}_{2}\right],\left[\mathbb{Z}_{d_{3}(n)}\right],\left[\mathbb{D}_{d_{2}(n)}\right]\right\} .
$$

Proof. Let $\Gamma=\mathbb{T} \cap\left(g \mathbb{D}_{n} g^{-1}\right)$. From decomposition (A-3), we only need to consider intersections

$$
\mathbb{Z}_{3}^{v t_{i}} \cap\left(g \mathbb{D}_{n} g^{-1}\right) \quad \text { and } \quad \mathbb{Z}_{2}^{e t_{j}} \cap\left(g \mathbb{D}_{n} g^{-1}\right),
$$

which have already been studied (see Lemma A.3).

Lemma A.7. We have

$$
[\mathbb{\mathbb { V }}] \odot[\mathbb{\mathbb { V }}]=\left\{[\mathbb{1}],\left[\mathbb{Z}_{2}\right],\left[\mathbb{D}_{2}\right],\left[\mathbb{Z}_{3}\right],[\mathbb{\mathbb { T }}]\right\} .
$$

Proof. Let $\Gamma=\mathbb{T} \cap\left(g \mathbb{\rrbracket} g^{-1}\right)$.

(1) If no axes match, then $\Gamma=\mathbb{1}$.

(2) If only one edge axis or one face axis from both configurations match, then $\Gamma \in\left[\mathbb{Z}_{2}\right]$ or $\left[\mathbb{Z}_{3}\right]$, respectively.

(3) If $g=\boldsymbol{Q}(\boldsymbol{k}, \pi / 2)$, then $\Gamma=\mathbb{D}_{2}$.

Octahedral subgroup. The group $\mathbb{D}$ is the (orientation-preserving) symmetry group of the cube $\mathscr{C}_{0}$ (see Figure 10) with vertices

$$
\left\{A_{i}\right\}_{i=1, \ldots, 8}=( \pm 1, \pm 1, \pm 1) .
$$

We have the decomposition [Ihrig and Golubitsky 1984]

$$
\mathbb{O}=\biguplus_{i=1}^{3} \mathbb{Z}_{4}^{f c_{i}} \uplus \biguplus_{j=1}^{4} \mathbb{Z}_{3}^{v c_{j}} \uplus \biguplus_{l=1}^{6} \mathbb{Z}_{2}^{e c_{l}}
$$

with vertices, edges, and faces axes respectively denoted $\boldsymbol{v} \boldsymbol{c}_{i}, \boldsymbol{e} \boldsymbol{c}_{j}$, and $\boldsymbol{f} \boldsymbol{c}_{j}$. For instance we have

$$
\boldsymbol{v} c_{1}:=\left(O A_{1}\right), \quad e c_{1}:=\left(O M_{1}\right), \quad f c_{1}:=(O I) .
$$

As an application of decomposition (A-4) and Lemma A.2, we obtain: 
Corollary A.8. Let $n \geq 2$ be some integer. Set

$$
d_{2}(n)=\operatorname{gcd}(n, 2), \quad d_{3}(n)=\operatorname{gcd}(n, 3),
$$

and

Then we have

$$
d_{4}(n)= \begin{cases}4 & \text { if } 4 \text { divide } n \\ 1 & \text { otherwise }\end{cases}
$$

$$
\left[\mathbb{Z}_{n}\right] \odot[\mathbb{O}]=\left\{[\mathbb{1}],\left[\mathbb{Z}_{d_{2}(n)}\right],\left[\mathbb{Z}_{d_{3}(n)}\right],\left[\mathbb{Z}_{d_{4}(n)}\right]\right\} .
$$

Corollary A.9. Let $n \geq 2$ be some integer. Set

$$
d_{2}(n)=\operatorname{gcd}(n, 2), \quad d_{3}(n)=\operatorname{gcd}(n, 3),
$$

and

$$
d_{4}(n)= \begin{cases}4 & \text { if } 4 \text { divides } n \\ 1 & \text { otherwise }\end{cases}
$$

Then we have

$$
\left[\mathbb{D}_{n}\right] \odot[\mathbb{O}]=\left\{[\mathbb{1}],\left[\mathbb{Z}_{2}\right],\left[\mathbb{Z}_{d_{3}(n)}\right],\left[\mathbb{Z}_{d_{4}(n)}\right],\left[\mathbb{D}_{d_{2}(n)}\right],\left[\mathbb{D}_{d_{3}(n)}\right],\left[\mathbb{D}_{d_{4}(n)}\right]\right\} .
$$

Proof. Using decomposition (A-4), we have to consider intersections

$$
\mathbb{D}_{n} \cap\left(g \mathbb{Z}_{4}^{f c_{i}} g^{-1}\right), \quad \mathbb{D}_{n} \cap\left(g \mathbb{Z}_{3}^{v c_{j}} g^{-1}\right), \quad \mathbb{D}_{n} \cap\left(g \mathbb{Z}_{2}^{e c_{l}} g^{-1}\right),
$$

which have already been studied in Lemma A.3.

Lemma A.10. We have

$$
[\mathbb{\square}] \odot[\mathbb{O}]=\left\{[\mathbb{1}],\left[\mathbb{Z}_{2}\right],\left[\mathbb{D}_{2}\right],\left[\mathbb{Z}_{3}\right],[\mathbb{\mathbb { T }}]\right\} .
$$

Proof. Let $\Gamma=\mathbb{O} \cap\left(g \llbracket g^{-1}\right)$. From decompositions (A-3)-(A-4) and Lemma A.2, we only have to consider intersections

$$
\mathbb{Z}_{4}^{f c_{i}} \cap\left(g \mathbb{Z}_{2}^{e t_{j}} g^{-1}\right), \quad \mathbb{Z}_{3}^{v c_{j}} \cap\left(g \mathbb{Z}_{3}^{v t_{i}} g^{-1}\right), \quad \mathbb{Z}_{2}^{e c_{l}} \cap\left(g \mathbb{Z}_{2}^{e t_{j}} g^{-1}\right) .
$$

Now, we always can find $g$ such that the intersection $\Gamma$ reduces to some subgroup conjugate to $\mathbb{1}, \mathbb{Z}_{2}$, or $\mathbb{Z}_{3}$, and taking $g=\boldsymbol{Q}(\boldsymbol{k}, \pi / 4)$, we get that $\Gamma$ is conjugate to $\mathbb{D}_{2}$, which achieves the proof.

Lemma A.11. We have

$$
[\mathbb{O}] \odot[\mathbb{O}]=\left\{[\mathbb{1}],\left[\mathbb{Z}_{2}\right],\left[\mathbb{D}_{2}\right],\left[\mathbb{Z}_{3}\right],\left[\mathbb{D}_{3}\right],\left[\mathbb{Z}_{4}\right],\left[\mathbb{D}_{4}\right],[\mathbb{O}]\right\} .
$$

Proof. Consider the subgroup $\Gamma=\mathbb{O} \cap\left(g \mathbb{O} g^{-1}\right) \subset \mathbb{O}$. From the poset in Figure 1, we deduce that the conjugacy class $[\Gamma]$ belong to the list

$$
\left\{[\mathbb{1}],\left[\mathbb{Z}_{2}\right],\left[\mathbb{D}_{2}\right],\left[\mathbb{Z}_{3}\right],\left[\mathbb{D}_{3}\right],\left[\mathbb{Z}_{4}\right],\left[\mathbb{D}_{4}\right],[\mathbb{\square}],[\mathbb{O}]\right\} .
$$

Now: 


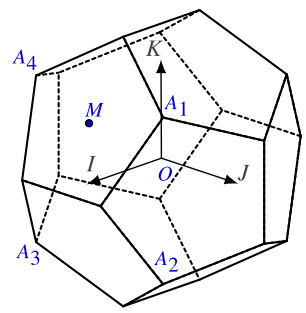

Figure 11. Dodecahedron $\mathscr{D}_{0}$.

(1) If $g$ fixes only one edge axis or one vertex axis, then $\Gamma \in\left[\mathbb{Z}_{2}\right]$ or $\Gamma \in\left[\mathbb{Z}_{3}\right]$, respectively.

(2) If $g=\boldsymbol{Q}(\boldsymbol{i} ; \pi / 6)$, only one face axis is fixed by $g$ and $\Gamma \in\left[\mathbb{Z}_{4}\right]$.

(3) If $g=\boldsymbol{Q}(\boldsymbol{i} ; \pi / 4), \Gamma \supset \mathbb{Z}_{4}^{\boldsymbol{i}} \uplus \mathbb{Z}_{2}^{\boldsymbol{k}}$ and thus $\Gamma \in\left[\mathbb{D}_{4}\right]$.

(4) If $g=\boldsymbol{Q}(\boldsymbol{k} ; \pi / 4) \circ \boldsymbol{Q}(\boldsymbol{i} ; \pi / 4), \Gamma \in\left[\mathbb{D}_{2}\right]$ with characteristic axes $g \boldsymbol{f} \boldsymbol{c}_{3}=\boldsymbol{e} \boldsymbol{c}_{6}$, $g e c_{1}=f c_{1}$, and $g e c_{2}=e c_{5}$.

(5) If $g=\boldsymbol{Q}\left(\boldsymbol{v} \boldsymbol{c}_{1}, \pi\right), \Gamma \in\left[\mathbb{D}_{3}\right]$ with $\boldsymbol{v} \boldsymbol{c}_{1}$ as the primary axis and $\boldsymbol{e c _ { 5 }}$ as the secondary axis of $\Gamma$.

(6) If $\Gamma \supset \mathbb{T}$, then $g$ fixes the three edge axes of the tetrahedron, and $g$ fixes the cube. In that case, $\Gamma=\mathbb{O}$.

Icosahedral subgroup. The group $\llbracket$ is the (orientation-preserving) symmetry group of the dodecahedron $\mathscr{D}_{0}$ (Figure 11), where we have

- twelve vertices $\left( \pm \phi, \pm \phi^{-1}, 0\right),\left( \pm \phi^{-1}, 0, \pm \phi\right),\left(0, \pm \phi, \pm \phi^{-1}\right), \phi$ being the gold number, or

- eight vertices $( \pm 1, \pm 1, \pm 1)$ of a cube.

We thus have the decomposition

$$
\mathbb{q}=\biguplus_{i=1}^{6} \mathbb{Z}_{5}^{f \boldsymbol{d}_{i}} \uplus \biguplus_{j=1}^{10} \mathbb{Z}_{3}^{\boldsymbol{v} \boldsymbol{d}_{j}} \uplus \biguplus_{l=1}^{15} \mathbb{Z}_{2}^{\boldsymbol{e} \boldsymbol{d}_{l}}
$$

with vertices, edges, and faces axes respectively denoted $\boldsymbol{v} \boldsymbol{d}_{i}, \boldsymbol{e d}{ }_{j}$, and $\boldsymbol{f} \boldsymbol{d}_{j}$. For instance we have

$$
\boldsymbol{v d _ { 1 }}:=\left(O A_{1}\right), \quad \boldsymbol{e d} d_{1}:=(O I), \quad f d_{1}:=(O M)
$$

where $M$ is the center of some face.

From decomposition (A-5) and Lemma A.2 we obtain the following corollary.

Corollary A.12. Let $n \geq 2$ be some integer. Set

$$
d_{2}:=\operatorname{gcd}(n, 2), \quad d_{3}:=\operatorname{gcd}(n, 3), \quad d_{5}:=\operatorname{gcd}(n, 5) .
$$


Then

$$
\left[\mathbb{Z}_{n}\right] \odot[\square]=\left\{[\mathbb{1}],\left[\mathbb{Z}_{d_{2}}\right],\left[\mathbb{Z}_{d_{3}}\right],\left[\mathbb{Z}_{d_{5}}\right]\right\} .
$$

Once again using decomposition (A-5) and the clips operation $\left[\mathbb{D}_{n}\right] \odot\left[\mathbb{Z}_{m}\right]$ in Lemma A.3 we get the following corollary.

Corollary A.13. Let $n \geq 2$ be some integer. Set

$$
d_{2}:=\operatorname{gcd}(n, 2) \quad d_{3}:=\operatorname{gcd}(n, 3), \quad d_{5}:=\operatorname{gcd}(n, 5) .
$$

Then

$$
\left[\mathbb{D}_{n}\right] \odot[\mathbb{0}]=\left\{[\mathbb{1}],\left[\mathbb{Z}_{2}\right],\left[\mathbb{Z}_{d_{3}}\right],\left[\mathbb{Z}_{d_{5}}\right],\left[\mathbb{D}_{d_{2}}\right],\left[\mathbb{D}_{d_{3}}\right],\left[\mathbb{D}_{d_{5}}\right]\right\} .
$$

Lemma A.14.

$$
[0] \odot[\mathbb{\square}]=\left\{[\mathbb{1}],\left[\mathbb{Z}_{2}\right],\left[\mathbb{Z}_{3}\right],[\mathbb{\mathbb { T }}]\right\} \text {. }
$$

Proof. Let $\Gamma=\llbracket \cap\left(g \llbracket g^{-1}\right)$. From decompositions (A-3)-(A-5) and Lemma A.2, we only have to consider intersections

$$
\mathbb{Z}_{3}^{v d_{j}} \cap\left(g \mathbb{Z}_{3}^{v t_{i}} g^{-1}\right), \quad \mathbb{Z}_{2}^{e d_{l}} \cap\left(g \mathbb{Z}_{2}^{e t_{j}} g^{-1}\right) .
$$

First, note that there always exists $g$ such that $\Gamma$ reduces to a subgroup conjugate to $\mathbb{1}, \mathbb{Z}_{2}$, or $\mathbb{Z}_{3}$. Now, if $\Gamma$ contains a subgroup conjugate to $\mathbb{D}_{2}$, then its three characteristic axes are edge axes of the dodecahedron: say $O x, O y$, and $O z$. In that case, $g$ fixes these three axes, and also the eight vertices of the cube $\mathscr{C}_{0}$. The subgroup $g \llbracket g^{-1}$ is thus the (orientation-preserving) symmetry group of a tetrahedron included in the dodecahedron $\mathscr{D}_{0}$. Then, $\Gamma \in[\mathbb{T}]$.

The next two lemmas are more involved.

Lemma A.15. $\quad[\mathbb{O}] \odot[\mathbb{0}]=\left\{[\mathbb{1}],\left[\mathbb{Z}_{2}\right],\left[\mathbb{Z}_{3}\right],\left[\mathbb{D}_{3}\right],[\mathbb{\mathbb { T }}]\right\}$.

Proof. Let $\Gamma=\llbracket \cap\left(g \mathbb{O} g^{-1}\right)$. From the poset in Figure 1, we deduce that the conjugacy class $[\Gamma]$ belongs to the list

$$
\left\{[\mathbb{1}],\left[\mathbb{Z}_{2}\right],\left[\mathbb{D}_{2}\right],\left[\mathbb{Z}_{3}\right],\left[\mathbb{D}_{3}\right],[\mathbb{\mathbb { T }}]\right\} .
$$

First, we can always find $g \in \operatorname{SO}(3)$ such that $\Gamma \in\left[\mathbb{Z}_{3}\right], \Gamma \in\left[\mathbb{Z}_{2}\right]$, or $\Gamma=\mathbb{1}$. Moreover, as in the proof of Lemma A.14, if $\Gamma$ contains a subgroup conjugate to $\mathbb{D}_{2}$, then $\Gamma \in[\mathbb{T}]$. Finally, we will exhibit some $g \in \operatorname{SO}(3)$ such that $\Gamma \in\left[\mathbb{D}_{3}\right]$. First, recall that

$$
A_{1}(1,1,1), \quad A_{2}(1,1,-1), \quad A_{4}(1,-1,1), \quad A_{5}(-1,-1,1)
$$

are common vertices of the cube $\mathscr{C}_{0}$ and dodecahedron $\mathscr{D}_{0}$. Now let $B_{2}\left(\phi^{-1}, 0,-\phi\right)$ be a vertex of the dodecahedron and $I_{2}$ or $I_{4}$ be the middle-point of $\left[B_{2} A_{2}\right]$ or $\left[A_{4} A_{5}\right]$, respectively (see Figure 12). 


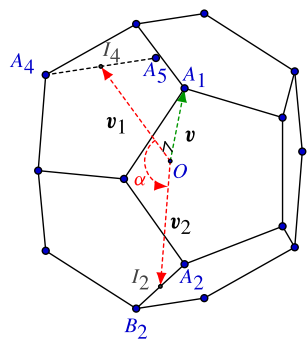

Figure 12. Rotation $g$ to obtain $\left[\mathbb{D}_{3}\right]$ in $[\mathbb{O}] \odot[\square]$.

Then, $a_{1}=\left(O I_{4}\right)$ and $a_{2}=\left(O I_{2}\right)$ are perpendicular axes to $a=\left(O A_{1}\right)$. Choose $\alpha$ such that $\boldsymbol{Q}(\boldsymbol{v}, \alpha) \boldsymbol{v}_{1}=\boldsymbol{v}_{2}$, with (see Figure 12)

$$
\boldsymbol{v}=\overrightarrow{O A_{1}}, \quad v_{1}=\overrightarrow{O I}_{4}, \quad v_{2}=\overrightarrow{O I}_{2},
$$

and set $g=\boldsymbol{Q}(\boldsymbol{v}, \alpha)$. From decompositions (A-4) and (A-5), we then deduce that $\square \cap\left(g \mathbb{O} g^{-1}\right)$ contains the subgroups

$$
\mathbb{Z}_{2}^{a_{2}} \cap\left(g \mathbb{Z}_{2}^{a_{1}} g^{-1}\right)=\mathbb{Z}_{2}^{a_{2}}, \quad \mathbb{Z}_{3}^{a} \cap,\left(g \mathbb{Z}_{3}^{a} g^{-1}\right)=\mathbb{Z}_{3}^{a} .
$$

Therefore, $\Gamma$ contains a subgroup conjugate to $\mathbb{D}_{3}$. Using a maximality argument (see poset in Figure 1), we must have $\Gamma \in\left[\mathbb{D}_{3}\right]$, and this concludes the proof.

Lemma A.16. $\quad[\square] \odot[\square]=\left\{[\mathbb{1}],\left[\mathbb{Z}_{2}\right],\left[\mathbb{Z}_{3}\right],\left[\mathbb{D}_{3}\right],\left[\mathbb{Z}_{5}\right],\left[\mathbb{D}_{5}\right],[\square]\right\}$.

Proof. Let $\Gamma=\llbracket \cap\left(g \llbracket g^{-1}\right)$. Considering the subclasses of [₫], we have to check the classes

$$
[\mathbb{\mathbb { V }}], \quad\left[\mathbb{D}_{3}\right], \quad\left[\mathbb{D}_{5}\right], \quad\left[\mathbb{D}_{2}\right], \quad\left[\mathbb{Z}_{3}\right], \quad\left[\mathbb{Z}_{5}\right], \quad\left[\mathbb{Z}_{2}\right] .
$$

First note that there exist rotations $g$ such that $\Gamma \in\left[\mathbb{Z}_{2}\right], \Gamma \in\left[\mathbb{Z}_{3}\right], \Gamma \in\left[\mathbb{Z}_{5}\right]$, or $\Gamma=\mathbb{1}$.

When $\Gamma$ contains a subgroup conjugate to $\mathbb{\mathbb { N }}$ or $\mathbb{D}_{2}$, using the same argument as in the proof of Lemma A.14, $g$ fixes all the dodecahedron vertices. In that case, $\Gamma=\square$.

We will now exhibit some $g \in \mathrm{SO}(3)$ such that $\Gamma \in\left[\mathbb{D}_{3}\right]$. Consider the dodecahedron $\mathscr{D}_{0}$ in Figure 11 and the points $A_{3}(1,-1,-1)$ and $B_{3}\left(\phi,-\phi^{-1}, 0\right)$. Let $I_{3}$ be the midpoint of $\left[A_{3} B_{3}\right]$ and $g$ be the order-2 rotation around $a_{1}:=\left(O A_{1}\right)$ (see Figure 13). Let

$$
b_{1}:=\left(O I_{3}\right), \quad b_{2}:=Q\left(a_{1}, \frac{2 \pi}{3}\right) b_{1}, \quad b_{3}:=Q\left(a_{1}, \frac{2 \pi}{3}\right) b_{2} .
$$

We check directly that $a_{1}$ and $b_{i}(i=1, \ldots, 3)$ are the only $g$-invariant characteristic axes of the dodecahedron. We then deduce from decomposition (A-5) that $\Gamma$ 


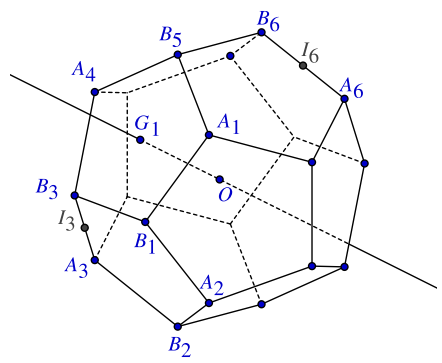

Figure 13. Rotation $g$ to obtain $\left[\mathbb{D}_{3}\right]$ or $\left[\mathbb{D}_{5}\right]$ in $[\square] \odot[\square]$.

reduces to

$$
\mathbb{Z}_{3}^{a_{1}} \uplus \biguplus_{i=1}^{3} \mathbb{Z}_{2}^{b_{i}} \in\left[\mathbb{D}_{3}\right] .
$$

In the same way, we can find $g \in \operatorname{SO}(3)$, such that $\Gamma \in\left[\mathbb{D}_{5}\right]$. Let

$$
B_{1}\left(\phi, \phi^{-1}, 0\right), \quad B_{5}\left(\phi^{-1}, 0, \phi\right), \quad B_{6}\left(-\phi^{-1}, 0, \phi\right), \quad A_{6}(-1,1,1)
$$

be vertices of the dodecahedron $\mathscr{D}_{0}$. Let $G_{1}$ be the center of pentagon $A_{1} B_{1} B_{3} A_{4} B_{5}$ (see Figure 13) and $I_{6}$ be the midpoint of $\left[B_{6} A_{6}\right]$. Let $g$ be the order-2 rotation around $f_{1}:=\left(O G_{1}\right)$ and set

$$
c_{1}:=\left(O I_{6}\right), \quad c_{k+1}:=Q\left(\overrightarrow{O A_{1}}, \frac{2 \pi}{5}\right) c_{k}, \quad 1 \leq k \leq 4 .
$$

Then we can check that $f_{1}$ and $c_{k}(k=1, \ldots, 5)$ are the only $g$-invariant characteristic axes of the dodecahedron. Using decomposition (A-5), we then deduce that $\Gamma \in\left[\mathbb{D}_{5}\right]$, which concludes the proof.

Infinite subgroups. The primary axis of both $\mathrm{SO}(2)$ and $\mathrm{O}(2)$ is defined as the $z$-axis, while any perpendicular axis to $(O z)$ is a secondary axis for $\mathrm{O}(2)$.

Clips operation between $\mathrm{SO}(2)$ or $\mathrm{O}(2)$ and finite subgroups are obtained using simple arguments on characteristic axes. The same holds for the clips $[\mathrm{SO}(2)] \odot$ $[\mathrm{SO}(2)]$. To compute $[\mathrm{O}(2)] \odot[\mathrm{O}(2)]$, consider the subgroup $\Gamma=\mathrm{O}(2) \cap\left(g \mathrm{O}(2) g^{-1}\right)$ for some $g \in \mathrm{SO}(3)$.

(1) If both primary axes are the same, then $\Gamma=\mathrm{O}(2)$.

(2) If the primary axis of $g \mathrm{O}(2) g^{-1}$ is in the $x y$-plane, then $\Gamma \in\left[\mathbb{D}_{2}\right]$.

(3) In all other cases, $\Gamma \in\left[\mathbb{Z}_{2}\right]$, where the primary axis of $\Gamma$ is perpendicular to the primary axes of $\mathrm{O}(2)$ and $g \mathrm{O}(2) g^{-1}$. 


\begin{tabular}{|ccc|}
\hline$\Gamma$ & $H$ & $L$ \\
\hline $\mathbb{Z}_{2}^{-}$ & $\mathbb{Z}_{2}$ & $\mathbb{1}$ \\
$\mathbb{Z}_{2 n}^{-}$ & $\mathbb{Z}_{2 n}$ & $\mathbb{Z}_{n}$ \\
$\mathbb{D}_{n}^{v}$ & $\mathbb{D}_{n}$ & $\mathbb{Z}_{n}$ \\
$\mathbb{D}_{2 n}^{h}$ & $\mathbb{D}_{2 n}$ & $\mathbb{D}_{n}$ \\
$\mathbb{O}^{-}$ & $\mathbb{O}$ & $\mathbb{1}$ \\
$\mathrm{O}(2)^{-}$ & $\mathrm{O}(2)$ & $\mathrm{SO}(2)$ \\
\hline
\end{tabular}

Table 3. Characteristic couples for type III subgroups.

\section{Appendix B: Proofs for $\mathbf{O}(3)$}

In this appendix, we provide the details about clips operations between type III closed $\mathrm{O}(3)$ subgroups. The proofs follow the same ideas that have been used for $\mathrm{SO}$ (3) closed subgroups (decomposition into simpler subgroups and discussion about their characteristic axes), but most of them are unfortunately more involved.

We first recall the general structure of type III subgroups $\Gamma$ of $\mathrm{O}(3)$ (see [Ihrig and Golubitsky 1984] for details). For each such subgroup $\Gamma$, there exist a couple $L \subset H$ of $\mathrm{SO}(3)$ subgroups such that $H=\pi(\Gamma)$, where

$$
\pi: g \in \mathrm{O}(3) \mapsto \operatorname{det}(g) g \in \mathrm{SO}(3)
$$

and $L=\mathrm{SO}(3) \cap \Gamma$ is an indexed 2 subgroup of $H$. These characteristic couples are detailed in Table 3 . Note that, for a given couple $(L, H), \Gamma$ can be recovered as $\Gamma=L \cap g L$, where $-g \in H \backslash L$.

In the following, we shall use the convention

$$
\mathbb{Z}_{1}^{\sigma}=\mathbb{Z}_{1}^{-}=\mathbb{D}_{1}^{v}=\mathbb{1}
$$

$\mathbb{Z}_{\mathbf{2 n}}^{-}$subgroups. Consider the couple $\mathbb{Z}_{n} \subset \mathbb{Z}_{2 n}(n>1)$ in Table 3 , where

$$
\mathbb{Z}_{2 n}=\left\{I, Q\left(k ; \frac{\pi}{n}\right), Q\left(k ; \frac{2 \pi}{n}\right), \ldots\right\}
$$

and let $\boldsymbol{r}_{n}:=\boldsymbol{Q}(\boldsymbol{k} ; \pi / n) \in \mathbb{Z}_{2 n} \backslash \mathbb{Z}_{n}$. Set

$$
\mathbb{Z}_{2 n}^{-}:=\mathbb{Z}_{n} \cup\left(-\boldsymbol{r}_{n} \mathbb{Z}_{n}\right)
$$

The primary axis of the subgroup $\mathbb{Z}_{2 n}^{-}$is defined as the $z$-axis.

Remark B.1. The subgroup $\mathbb{Z}_{2}^{-}$is generated by $-\boldsymbol{Q}(\boldsymbol{k}, \pi)$, which is the reflection through the $x y$-plane. If $\sigma_{b}$ is the reflection through the plane with normal axis $b$, then $\mathbb{Z}_{2}^{\sigma_{b}}:=\left\{e, \sigma_{b}\right\}$, which is conjugate to $\mathbb{Z}_{2}^{-}$.

We have the following lemma. 
Lemma B.2. Let $m, n \geq 2$ be two integers. Set $d:=\operatorname{gcd}(n, m)$ and

$$
\boldsymbol{r}_{n}:=Q\left(\boldsymbol{k} ; \frac{\pi}{n}\right), \quad \boldsymbol{r}_{m}:=Q\left(\boldsymbol{k} ; \frac{\pi}{m}\right) .
$$

The intersection $\left(-\boldsymbol{r}_{n} \mathbb{Z}_{n}\right) \cap\left(-\boldsymbol{r}_{m} \mathbb{Z}_{m}\right)$ does not reduce to $\varnothing$ if and only if $m / d$ and $n / d$ are odd. In such a case, we have

$$
\left(-\boldsymbol{r}_{n} \mathbb{Z}_{n}\right) \cap\left(-\boldsymbol{r}_{m} \mathbb{Z}_{m}\right)=-\boldsymbol{r}_{d} \mathbb{Z}_{d}, \quad \boldsymbol{r}_{d}=\boldsymbol{Q}\left(\boldsymbol{k} ; \frac{\pi}{d}\right) .
$$

Proof. The intersection $\left(-\boldsymbol{r}_{n} \mathbb{Z}_{n}\right) \cap\left(-\boldsymbol{r}_{m} \mathbb{Z}_{m}\right)$ differs from $\varnothing$, if and only if there exist integers $i, j$ such that

$$
\frac{2 i+1}{n} \pi=\frac{2 j+1}{m} \pi, \quad 2 i+1 \leq 2 n, \quad 2 j+1 \leq 2 m .
$$

Let $n=d n_{1}$ and $m=d m_{1}$. The above equation can then be recast as $(2 i+1) m_{1}=$ $(2 j+1) n_{1}$, so that

$$
2 i+1=p n_{1} \quad \text { and } \quad 2 j+1=p m_{1} .
$$

Thus, $m_{1}$ and $n_{1}$ are necessarily odd, in which case (recall that $\mathbb{Z}_{1}=\mathbb{1}$ )

$$
\left(-\boldsymbol{r}_{n} \mathbb{Z}_{n}\right) \cap\left(-\boldsymbol{r}_{n} \mathbb{Z}_{m}\right)=-\boldsymbol{r}_{d} \mathbb{Z}_{d}, \quad \boldsymbol{r}_{d}=\boldsymbol{Q}\left(\boldsymbol{k} ; \frac{\pi}{d}\right) .
$$

Corollary B.3. Let $m, n \geq 1$ be two integers. Set $d:=\operatorname{gcd}(n, m)$. Then we have

$$
\left[\mathbb{Z}_{2 n}^{-}\right] \odot\left[\mathbb{Z}_{2 m}^{-}\right]= \begin{cases}\left\{[\mathbb{1}],\left[\mathbb{Z}_{2 d}^{-}\right]\right\} & \text {if } n / d \text { and } m / d \text { are odd }, \\ \left\{[\mathbb{1}],\left[\mathbb{Z}_{d}\right]\right\} & \text { otherwise. }\end{cases}
$$

Proof. Note first that all intersections reduce to $\mathbb{1}$ when the characteristic axes don't match, so we only have to consider the situation where they match. Now, by (B-1), we only have to consider the intersection

$$
\mathbb{Z}_{2 n}^{-} \cap \mathbb{Z}_{2 m}^{-}=\left(\mathbb{Z}_{n} \cap \mathbb{Z}_{m}\right) \cup\left(\left(-\boldsymbol{r}_{n} \mathbb{Z}_{n}\right) \cap\left(-\boldsymbol{r}_{m} \mathbb{Z}_{m}\right)\right) .
$$

By Lemma A.2, $\mathbb{Z}_{n} \cap \mathbb{Z}_{m}=\mathbb{Z}_{d}$ and we directly conclude with Lemma B.2.

$\mathbb{D}_{n}^{v}$ subgroups. Consider the couple $\mathbb{Z}_{n} \subset \mathbb{D}_{n}$ in Table 3 . Recall that $\mathbb{D}_{n}$ contains $\mathbb{Z}_{n}$ and all the second-order rotations about the $b_{j}$ axes (see (A-2) and Figure 9). Set

$$
\mathbb{D}_{n}^{v}:=\mathbb{Z}_{n} \uplus \biguplus_{j=1}^{n} \mathbb{Z}_{2}^{\sigma_{b_{j}}} .
$$

Given $g \in \mathrm{O}(3)$, the primary axis of $g \mathbb{D}_{n}^{v} g^{-1}$ is $g(O z)$, and its secondary axes are $g b_{j}$. 
Lemma B.4. Let $n \geq 2$ and $m \geq 1$ be two integers. Set $d=\operatorname{gcd}(n, m)$ and

Then, we have

$$
i(m):=3-\operatorname{gcd}(2, m)= \begin{cases}1 & \text { if } m \text { is even }, \\ 2 & \text { if } m \text { is odd }\end{cases}
$$

$$
\left[\mathbb{D}_{n}^{v}\right] \odot\left[\mathbb{Z}_{2 m}^{-}\right]=\left\{\mathbb{1},\left[\mathbb{Z}_{i(m)}^{-}\right],\left[\mathbb{Z}_{d}\right]\right\} .
$$

Proof. Let $\Gamma:=\mathbb{D}_{n}^{v} \cap\left(g \mathbb{Z}_{2 m}^{-} g^{-1}\right)$ and

$$
\mathbb{Z}_{2 m}^{-}=\mathbb{Z}_{m} \cup\left(-\boldsymbol{r}_{m} \mathbb{Z}_{m}\right), \quad \boldsymbol{r}_{m}=\boldsymbol{Q}\left(\boldsymbol{k} ; \frac{\pi}{m}\right) .
$$

(1) If both primary axes of $\mathbb{D}_{n}^{v}$ and $g \mathbb{Z}_{2 m}^{-} g^{-1}$ (generated by $g \boldsymbol{k}$ ) match, then by decomposition (B-2) and Lemma A.2, $\Gamma$ reduces to $\mathbb{Z}_{n} \cap \mathbb{Z}_{m}=\mathbb{Z}_{d}$.

(2) If the primary axis of $g \mathbb{Z}_{2 m}^{-} g^{-1}$ matches with a secondary axis of $\mathbb{D}_{n}^{v}$, say $(O x)$, then $\Gamma$ reduces to $\mathbb{Z}_{2}^{\sigma_{b_{0}}} \cap\left(g \mathbb{Z}_{2 m}^{-} g^{-1}\right)$. Such an intersection has already been studied in the clips operation $\left[\mathbb{Z}_{2}^{-}\right] \odot\left[\mathbb{Z}_{2 m}^{-}\right]$(see Corollary B.3).

(3) Otherwise, $\Gamma=\mathbb{1}$, which concludes the proof.

Lemma B.5. Let $m, n \geq 2$ be two integers and $d=\operatorname{gcd}(n, m)$. Then we have

$$
\left[\mathbb{D}_{n}^{v}\right] \odot\left[\mathbb{D}_{m}^{v}\right]=\left\{\mathbb{1},\left[\mathbb{Z}_{2}^{-}\right],\left[\mathbb{D}_{d}^{v}\right],\left[\mathbb{Z}_{d}\right]\right\} .
$$

Proof. Only two cases need to be considered.

(1) If the primary axes of $\mathbb{D}_{n}^{v}$ and $g \mathbb{D}_{m}^{v} g^{-1}$ do not match, then we get $\mathbb{1}$.

(2) If they have the same primary axis, by decomposition (B-2), we have to consider the intersections

$$
\mathbb{Z}_{n} \cap \mathbb{Z}_{m}, \quad \mathbb{Z}_{2}^{\sigma_{b_{j}}} \cap \mathbb{Z}_{2}^{\sigma_{b_{k}^{\prime}}},
$$

which reduce to

$$
\mathbb{Z}_{d} \uplus \mathbb{Z}_{2}^{\sigma_{c_{l}}},
$$

where $c_{l}$ are the common secondary axes of the two subgroups. Then we get either $\mathbb{Z}_{d}, \mathbb{D}_{d}^{v}$, or a subgroup conjugate to $\mathbb{Z}_{2}^{-}$(when $d=1$ and $b_{0}=b_{0}^{\prime}$ ), which concludes the proof.

$\mathbb{D}_{2 n}^{h}$ subgroups. Consider the couple $\mathbb{D}_{n} \subset \mathbb{D}_{2 n}$ in Table 3. For $j=0, \ldots, n-1$, let $p_{j}$ be the axis generated by

$$
\boldsymbol{v}_{j}:=Q\left(k ; \frac{j \pi}{n}\right) \cdot \boldsymbol{i},
$$

(see Figure 14) and $q_{j}$ the axis generated by

$$
\boldsymbol{w}_{j}:=\boldsymbol{Q}\left(\boldsymbol{k} ; \frac{(2 j+1) \pi}{2 n}\right) \cdot \boldsymbol{i} .
$$



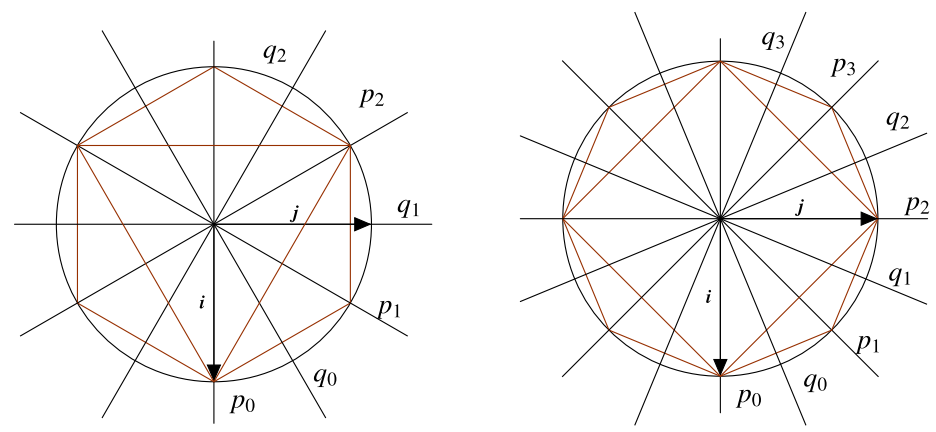

Figure 14. Left: characteristic axes for $\mathbb{D}_{6} \supset \mathbb{D}_{3}$. Right: characteristic axes for $\mathbb{D}_{8} \supset \mathbb{D}_{4}$.

Set

$$
\mathbb{D}_{n}=\left\{\mathbb{1}, \boldsymbol{Q}\left(\boldsymbol{k} ; \frac{2 \pi}{n}\right), \boldsymbol{Q}\left(\boldsymbol{k} ; \frac{4 \pi}{n}\right), \ldots, \boldsymbol{Q}\left(\boldsymbol{v}_{0} ; \pi\right), \boldsymbol{Q}\left(\boldsymbol{v}_{1} ; \pi\right), \ldots\right\},
$$

and

$$
-\boldsymbol{r}_{n} \mathbb{D}_{n}=\left\{-\boldsymbol{Q}\left(\boldsymbol{k} ; \frac{\pi}{n}\right),-\boldsymbol{Q}\left(\boldsymbol{k} ; \frac{3 \pi}{n}\right), \ldots,-\boldsymbol{Q}\left(\boldsymbol{w}_{0} ; \pi\right),-\boldsymbol{Q}\left(\boldsymbol{w}_{1} ; \pi\right), \ldots\right\},
$$

where $\boldsymbol{r}_{n}=\boldsymbol{Q}(\boldsymbol{k} ; \pi / n)$. We define

$$
\mathbb{D}_{2 n}^{h}:=\mathbb{D}_{n} \cup\left(-\boldsymbol{r}_{n} \mathbb{D}_{n}\right),
$$

which decomposes as

$$
\mathbb{D}_{2 n}^{h}=\mathbb{Z}_{2 n}^{-} \uplus \biguplus_{j=0}^{n-1} \mathbb{Z}_{2}^{p_{j}} \uplus \biguplus_{j=0}^{n-1} \mathbb{Z}_{2}^{\sigma_{q_{j}}} .
$$

Note that in this decomposition, there are $n$ subgroups conjugate to $\mathbb{Z}_{2}$ and $n$ others conjugate to $\mathbb{Z}_{2}^{-}$. The $z$-axis and $x$-axis are said to be the primary and secondary axes of $\mathbb{D}_{2 n}^{h}$, respectively. For each $g \in \mathrm{O}(3)$, the primary and secondary axes of the subgroup $g \mathbb{D}_{2 n}^{h} g^{-1}$ are generated by $g \boldsymbol{k}$ and by $g \boldsymbol{i}$, respectively.

Lemma B.6. Let $m, n \geq 2$ be two integers. Set $d=\operatorname{gcd}(n, m), d_{2}(m)=\operatorname{gcd}(m, 2)$, and

$$
i(m)= \begin{cases}1 & \text { if } m \text { is even } \\ 2 & \text { otherwise }\end{cases}
$$

Then:

- If $n / d$ or $m / d$ is even, we have

$$
\left[\mathbb{D}_{2 n}^{h}\right] \odot\left[\mathbb{Z}_{2 m}^{-}\right]=\left\{\mathbb{1},\left[\mathbb{Z}_{d_{2}(m)}\right],\left[\mathbb{Z}_{i(m)}^{-}\right],\left[\mathbb{Z}_{d}\right]\right\} .
$$


- If $n / d$ and $m / d$ are odd, we have

$$
\left[\mathbb{D}_{2 n}^{h}\right] \odot\left[\mathbb{Z}_{2 m}^{-}\right]=\left\{\mathbb{1},\left[\mathbb{Z}_{d_{2}(m)}\right],\left[\mathbb{Z}_{i(m)}^{-}\right],\left[\mathbb{Z}_{2 d}^{-}\right]\right\} .
$$

Proof. First of all, if no characteristic axes of $\mathbb{D}_{2 n}^{h}$ and $g \mathbb{Z}_{2 m}^{-} g^{-1}$ match, then their intersection reduces to $\mathbb{1}$.

We now have to consider three cases:

(1) The first case is when $\mathbb{D}_{2 n}^{h}$ and $g \mathbb{Z}_{2 m}^{-} g^{-1}$ have the same primary axis. Then, using decompositions (B-4) and (B-1), we only have to consider the intersection

$$
\mathbb{Z}_{2 n}^{-} \cap \mathbb{Z}_{2 m}^{-}
$$

This has already been studied in the clips operation $\left[\mathbb{Z}_{2 n}^{-}\right] \odot\left[\mathbb{Z}_{2 m}^{-}\right]$in Corollary B.3, leading to the conjugacy class $\left[\mathbb{Z}_{d}\right]$ or $\left[\mathbb{Z}_{2 d}^{-}\right]$.

(2) The second one is when some secondary axis $p_{j}$ (say $p_{0}$ ) matches with the primary axis of $g \mathbb{Z}_{2 m}^{-} g^{-1}$; then we only have to consider intersection

$$
\mathbb{Z}_{2}^{p_{0}} \cap\left(g \mathbb{Z}_{2 m}^{-} g^{-1}\right)=\mathbb{Z}_{2}^{p_{0}} \cap\left(g \mathbb{Z}_{m} g^{-1}\right)
$$

leading to $\mathbb{Z}_{2}^{p_{0}}$ if $m$ is even.

(3) Finally, we have to consider the case when the primary axis of $g \mathbb{Z}_{2 m}^{-} g^{-1}$ is $q_{0}$. In that case the problem reduces to the intersection

$$
\mathbb{Z}_{2}^{\sigma_{q_{0}}} \cap\left(g \mathbb{Z}_{2 m}^{-} g^{-1}\right)
$$

leading to the conjugacy class $\left[\mathbb{Z}_{2}^{-}\right]$for odd $m$ (see Corollary B.3).

The cases $\left[\mathbb{D}_{2 n}^{h}\right] \odot\left[\mathbb{D}_{n}^{v}\right]$ and $\left[\mathbb{D}_{2 n}^{h}\right] \odot\left[\mathbb{D}_{2 m}^{h}\right]$ are more involved. We start by formulating the following lemma, without proof (see Figure 9 for an example).

Lemma B.7. If $n$ is even, then there exist $p_{k} \perp p_{l}$ and $q_{r} \perp q_{s}$, where no axes $p_{i}, q_{j}$ are perpendicular. If $n$ is odd, there exist $p_{i} \perp q_{j}$ and no axes $p_{k}, p_{l}$ nor $q_{r}, q_{s}$ are perpendicular.

Lemma B.8. Let $m, n \geq 2$ be two integers. Set $d_{2}(m):=\operatorname{gcd}(m, 2)$,

$$
i(m, n):= \begin{cases}2 & \text { if } m \text { is even and } n \text { is odd }, \\ 1 & \text { otherwise }\end{cases}
$$

and

$$
i(m):=3-\operatorname{gcd}(2, m)= \begin{cases}1 & \text { if } m \text { is even } \\ 2 & \text { if } m \text { is odd }\end{cases}
$$

Then we have

$$
\left[\mathbb{D}_{2 n}^{h}\right] \odot\left[\mathbb{D}_{m}^{v}\right]=\left\{[\mathbb{1}],\left[\mathbb{Z}_{i(m)}^{-}\right],\left[\mathbb{Z}_{d_{2}(m)}\right],\left[\mathbb{D}_{i(m, n)}^{v}\right],\left[\mathbb{Z}_{d}\right],\left[\mathbb{D}_{d}^{v}\right]\right\} .
$$


Proof. The only nontrivial cases are when $g \mathbb{D}_{m}^{v} g^{-1}$ and $\mathbb{D}_{2 n}^{h}$ have no matching characteristic axes. Now we have to distinguish whether the principal axis $a$ of $g \mathbb{D}_{m}^{v} g^{-1}$ is $(O z)$ or not:

(1) Let's first suppose that $a=(\mathrm{Oz})$. In that case, we need to compute the intersection $\mathbb{D}_{2 n}^{h} \cap \mathbb{D}_{m}^{v}$. From (B-2) and (B-4), this reduces to studying the three intersections

$$
\mathbb{Z}_{2 n}^{-} \cap \mathbb{Z}_{m}, \quad \mathbb{Z}_{2 n}^{-} \cap \mathbb{Z}_{2}^{\sigma_{b_{j}}}, \quad \mathbb{Z}_{2}^{\sigma_{q_{k}}} \cap \mathbb{Z}_{2}^{\sigma_{b_{j}}} .
$$

Now:

(a) The first intersection, $\mathbb{Z}_{2 n}^{-} \cap \mathbb{Z}_{m}$, reduces to $\mathbb{Z}_{n} \cap \mathbb{Z}_{m}=\mathbb{Z}_{d}$ (from (B-1) and Lemma A.2).

(b) The second one, $\mathbb{Z}_{2 n}^{-} \cap \mathbb{Z}_{2}^{\sigma_{b_{j}}}$, reduces to $\mathbb{1}$, since primary axes of $\mathbb{Z}_{2 n}^{-}$and $\mathbb{Z}_{2}^{\sigma_{b_{j}}}$ (conjugate to $\mathbb{Z}_{2}^{-}$) do not match.

(c) The last one, $\mathbb{Z}_{2}^{\sigma_{q_{k}}} \cap \mathbb{Z}_{2}^{\sigma_{b_{j}}}$, can reduce to some $\mathbb{Z}_{2}^{\sigma_{q_{k}}}$ if $b_{0}=q_{0}$. In that case, $\mathbb{D}_{2 n}^{h} \cap \mathbb{D}_{m}^{v}$ contains $\mathbb{Z}_{d}$ and some $\mathbb{Z}_{2}^{\sigma_{q_{k}}}$, which generate $\mathbb{D}_{d}^{v}$ (see (B-2)).

This first case thus leads to $\mathbb{Z}_{d}$ or $\mathbb{D}_{d}^{v}$.

(2) Consider now the case when $a \neq(O z)$. Thus, the intersections to be considered are

$$
\mathbb{Z}_{2 n}^{-} \cap \mathbb{Z}_{2}^{\sigma_{b_{0}^{\prime}}}, \quad \mathbb{Z}_{2}^{p_{j}} \cap \mathbb{Z}_{m}^{a}, \quad \mathbb{Z}_{2}^{\sigma_{q_{k}}} \cap \mathbb{Z}_{2}^{\sigma_{b_{j}^{\prime}}},
$$

where the $b_{j}^{\prime}$ axes are the secondary axes of $g \mathbb{D}_{m}^{v} g^{-1}$. Now:

(a) First suppose that $a=p_{0}$ (for instance) and all the other axes are different. Then, $\mathbb{Z}_{2}^{p_{j}} \cap \mathbb{Z}_{m}^{a}=\mathbb{Z}_{d_{2}(m)}^{a}$.

(b) Suppose now that $a=p_{0}$ and $b^{\prime} j=q_{k}$ for some couple $(k, j)$, so that $\mathbb{Z}_{2}^{\sigma_{q_{k}}} \cap \mathbb{Z}_{2}^{\sigma_{b_{j}^{\prime}}}=\mathbb{Z}_{2}^{\sigma_{q_{k}}}$. As $q_{k} \perp p_{0}$, we deduce from Lemma B.7 that $n$ is odd. All depend on the parity of $m$ : if $m$ is even, then $\Gamma$ contains $\mathbb{Z}_{2}^{p_{0}}$ and $\mathbb{Z}_{2}^{\sigma_{q_{k}}}$, and we obtain some subgroup conjugate to $\mathbb{D}_{2}^{v}$. If $m$ is odd, then $\Gamma$ reduces to $\mathbb{Z}_{2}^{\sigma_{q_{k}}}$, which is conjugate to $\mathbb{Z}_{2}^{-}$.

(c) Finally, suppose that $a \neq p_{j}$ for all $j$ (and recall that $a \neq(O z)$ ), so that the intersections to be considered are

$$
\mathbb{Z}_{2 n}^{-} \cap \mathbb{Z}_{2}^{\sigma_{b_{0}^{\prime}}}, \quad \mathbb{Z}_{2}^{\sigma_{q_{k}}} \cap \mathbb{Z}_{2}^{\sigma_{b_{j}^{\prime}}} .
$$

We thus only obtain some subgroups already considered in the previous cases, which concludes the proof.

Lemma B.9. Let $m, n \geq 2$ be two integers. Set $d=\operatorname{gcd}(n, m)$ and

$$
\Delta=\left[\mathbb{D}_{2 n}^{h}\right] \odot\left[\mathbb{D}_{2 m}^{h}\right] .
$$

Then $[\mathbb{1}] \subset \Delta$ and: 
- For every integer $d$ :

- If $m$ and $n$ are even, then $\Delta \supset\left\{\left[\mathbb{Z}_{2}\right],\left[\mathbb{D}_{2}\right]\right\}$.

- If $m$ and $n$ are odd, then $\Delta \supset\left\{\left[\mathbb{Z}_{2}^{-}\right]\right\}$.

- Otherwise, $\Delta \supset\left\{\left[\mathbb{Z}_{2}\right],\left[\mathbb{D}_{2}^{v}\right]\right\}$.

- If $d=1$, then:

- If $m$ and $n$ are odd, then $\Delta \supset\left\{\left[\mathbb{D}_{2}^{v}\right]\right\}$.

- Otherwise, $m$ or $n$ is even and $\Delta \supset\left\{\left[\mathbb{Z}_{2}\right],\left[\mathbb{Z}_{2}^{-}\right]\right\}$.

- If $d \neq 1$, then:

- If $m / d$ and $n / d$ are odd, then $\Delta \supset\left\{\left[\mathbb{Z}_{2 d}^{-}\right],\left[\mathbb{D}_{2 d}^{h}\right]\right\}$.

- Otherwise, $m / d$ or $n / d$ is even and $\Delta \supset\left\{\left[\mathbb{Z}_{d}\right],\left[\mathbb{D}_{d}\right],\left[\mathbb{D}_{d}^{v}\right]\right\}$.

Sketch of the proof. We consider decomposition (B-3). If no characteristic axes $\mathbb{D}_{2 n}^{h}$ and $g \mathbb{D}_{2 m}^{h} g^{-1}$ match, then their intersection reduces to $\mathbb{1}$. Otherwise, from (B-3) it reduces to

$$
\begin{array}{lll}
\mathbb{Z}_{2 n}^{-} \cap\left(g \mathbb{Z}_{2 m}^{-} g^{-1}\right), & \mathbb{Z}_{2 n}^{-} \cap\left(g \mathbb{Z}_{2}^{\sigma_{q_{k}^{\prime}}} g^{-1}\right), & \mathbb{Z}_{2}^{p_{j}} \cap\left(g \mathbb{Z}_{2}^{p_{k}^{\prime}} g^{-1}\right), \\
\mathbb{Z}_{2}^{\sigma_{q_{j}}} \cap\left(g \mathbb{Z}_{2 m}^{-} g^{-1}\right), & \mathbb{Z}_{2}^{\sigma_{q_{j}}} \cap\left(g \mathbb{Z}_{2}^{\sigma_{k}^{\prime}} g^{-1}\right), &
\end{array}
$$

where all $\mathbb{Z}_{2}^{\sigma_{q_{j}}}, \mathbb{Z}_{2}^{\sigma_{q_{k}^{\prime}}}$ are subgroups conjugate to $\mathbb{Z}_{2}^{-}$.

Now all these intersections have already been studied in the clips operation $\left[\mathbb{Z}_{2 r}^{-}\right] \odot\left[\mathbb{Z}_{2 s}^{-}\right]$. We can thus use Corollary B.3, argue on the characteristic axes, and use Lemma B.7 to conclude the proof in each case.

$\mathbb{O}^{-}$subgroup. Consider the couple $\mathbb{T} \subset \mathbb{O}$ in Table 3 and the decompositions

$$
\mathbb{O}=\biguplus_{i=1}^{3} \mathbb{Z}_{4}^{f c_{i}} \uplus \biguplus_{j=1}^{4} \mathbb{Z}_{3}^{v c_{j}} \uplus \biguplus_{l=1}^{6} \mathbb{Z}_{2}^{e c_{l}}
$$

and

$$
\mathbb{T}=\biguplus_{j=1}^{4} \mathbb{Z}_{3}^{v t_{j}} \uplus \mathbb{Z}_{2}^{e t_{1}} \uplus \mathbb{Z}_{2}^{e t_{2}} \uplus \mathbb{Z}_{2}^{e t_{3}}, \quad \mathbb{Z}_{2}^{e t_{i}} \subset \mathbb{Z}_{4}^{f c_{i}}, i=1,2,3 .
$$

This leads (see [Ihrig and Golubitsky 1984] for details) to the decomposition

$$
\mathbb{O}^{-}:=\biguplus_{i=1}^{3}\left(\mathbb{Z}_{4}^{f c_{i}}\right)^{-} \uplus \biguplus_{j=1}^{4} \mathbb{Z}_{3}^{v c_{j}} \uplus \biguplus_{l=1}^{6} \mathbb{Z}_{2}^{\sigma_{e c_{l}}},
$$

where $\left(\mathbb{Z}_{4}^{f \boldsymbol{c}_{i}}\right)^{-}$is the subgroup conjugate to $\mathbb{Z}_{4}^{-}$with $\boldsymbol{f} \boldsymbol{c}_{i}$ as primary axis. Note also that $\mathbb{Z}_{2}^{\sigma_{e_{l}}}$ are subgroups conjugate to $\mathbb{Z}_{2}^{-}$with $\boldsymbol{e} \boldsymbol{c}_{l}$ as primary axis.

Using this decomposition (B-5) and those of type III closed $\mathrm{O}(3)$ subgroups previously mentioned directly lead to the following corollaries. 
Corollary B.10. Let $n \geq 2$ be an integer, and set $d_{2}(n)=\operatorname{gcd}(n, 2)$ and $d_{3}(n)=$ $\operatorname{gcd}(3, n)$. Then:

- If $n$ is odd, we have

$$
\left[\mathbb{O}^{-}\right] \odot\left[\mathbb{Z}_{2 n}^{-}\right]=\left\{[\mathbb{1}],\left[\mathbb{Z}_{2}^{-}\right],\left[\mathbb{Z}_{d_{3}(n)}\right]\right\} .
$$

- If $n=2+4 k$ for $k \in \mathbb{N}$, we have

$$
\left[\mathbb{O}^{-}\right] \odot\left[\mathbb{Z}_{2 n}^{-}\right]=\left\{[\mathbb{1}],\left[\mathbb{Z}_{4}^{-}\right],\left[\mathbb{Z}_{d_{3}(n)}\right]\right\} .
$$

- If $n$ is even and 4 does not divide $n$, we have

$$
\left[\mathbb{O}^{-}\right] \odot\left[\mathbb{Z}_{2 n}^{-}\right]=\left\{[\mathbb{1}],\left[\mathbb{Z}_{2}\right],\left[\mathbb{Z}_{d_{3}(n)}\right]\right\} .
$$

Moreover, in all cases, we have

$$
\left[\mathbb{O}^{-}\right] \odot\left[\mathbb{D}_{n}^{v}\right]=\left\{[\mathbb{1}],\left[\mathbb{Z}_{2}^{-}\right],\left[\mathbb{Z}_{d_{3}(n)}\right],\left[\mathbb{D}_{d_{3}(n)}^{v}\right],\left[\mathbb{Z}_{d_{2}(n)}\right],\left[\mathbb{D}_{d_{2}(n)}^{v}\right]\right\} .
$$

Corollary B.11. Let $n \geq 2$ be an integer and $d_{3}(n):=\operatorname{gcd}(n, 3)$.

- If $n$ is even and $n=2+4 k$ for $k \in \mathbb{N}$, then we have

$$
\left[\mathbb{O}^{-}\right] \odot\left[\mathbb{D}_{2 n}^{h}\right]=\left\{[\mathbb{1}],\left[\mathbb{Z}_{4}^{-}\right],\left[\mathbb{D}_{4}^{h}\right],\left[\mathbb{Z}_{d_{3}(n)}\right],\left[\mathbb{D}_{d_{3}(n)}^{v}\right]\right\} .
$$

- If $n$ is even and 4 divides $n$, then we have

$$
\left[\mathbb{O}^{-}\right] \odot\left[\mathbb{D}_{2 n}^{h}\right]=\left\{[\mathbb{1}],\left[\mathbb{Z}_{2}\right],\left[\mathbb{D}_{2}\right],\left[\mathbb{D}_{2}^{v}\right],\left[\mathbb{Z}_{d_{3}(n)}\right],\left[\mathbb{D}_{d_{3}(n)}^{v}\right]\right\} .
$$

- If $n$ is odd, then we have

$$
\left[\mathbb{O}^{-}\right] \odot\left[\mathbb{D}_{2 n}^{h}\right]=\left\{[\mathbb{1}],\left[\mathbb{Z}_{2}\right],\left[\mathbb{Z}_{2}^{-}\right],\left[\mathbb{D}_{2}\right],\left[\mathbb{D}_{2}^{v}\right],\left[\mathbb{Z}_{d_{3}(n)}\right],\left[\mathbb{D}_{d_{3}(n)}^{v}\right]\right\} .
$$

Corollary B.12. $\left[\mathbb{O}^{-}\right] \odot\left[\mathbb{O}^{-}\right]=\left\{[\mathbb{1}],\left[\mathbb{Z}_{2}^{-}\right],\left[\mathbb{Z}_{4}^{-}\right],\left[\mathbb{Z}_{3}\right]\right\}$.

O(2) ${ }^{-}$subgroup. Consider the couple $\mathrm{SO}(2) \subset \mathrm{O}(2)$ in Table 3 and set

$$
\mathrm{O}(2)^{-}:=\mathrm{SO}(2) \uplus \biguplus_{v \subset x y \text {-plane }} \mathbb{Z}_{2}^{\sigma_{v}} .
$$

As $\mathbb{Z}_{2}^{\sigma_{v}}$ are subgroups conjugate to $\mathbb{Z}_{2}^{-}$, previous results on the clips operation of $\left[\mathbb{Z}_{2}^{-}\right]$and type III subgroups except $\left[\mathrm{O}(2)^{-}\right]$leads to the following lemma.

Lemma B.13. Let $n \geq 2$ be some integer. Set $d_{2}(n):=\operatorname{gcd}(2, n)$ and

$$
i(n):=3-\operatorname{gcd}(2, n)= \begin{cases}1 & \text { if } n \text { is even }, \\ 2 & \text { if } n \text { is odd }\end{cases}
$$


Then, we have

$$
\begin{aligned}
{\left[\mathrm{O}(2)^{-}\right] \odot\left[\mathbb{Z}_{2 n}^{-}\right] } & =\left\{[\mathbb{1}],\left[\mathbb{Z}_{i(n)}^{-}\right],\left[\mathbb{Z}_{n}\right]\right\}, \\
{\left[\mathrm{O}(2)^{-}\right] \odot\left[\mathbb{D}_{n}^{v}\right] } & =\left\{[\mathbb{1}],\left[\mathbb{Z}_{2}^{-}\right],\left[\mathbb{D}_{n}^{v}\right]\right\}, \\
{\left[\mathrm{O}(2)^{-}\right] \odot\left[\mathbb{D}_{2 n}^{h}\right] } & =\left\{[\mathbb{1}],\left[\mathbb{Z}_{d_{2}(n)}\right],\left[\mathbb{Z}_{2}^{-}\right],\left[\mathbb{D}_{i(n)}^{v}\right],\left[\mathbb{D}_{n}^{v}\right]\right\}, \\
{\left[\mathrm{O}(2)^{-}\right] \odot\left[\mathbb{O}^{-}\right] } & =\left\{[\mathbb{1}],\left[\mathbb{Z}_{2}^{-}\right],\left[\mathbb{D}_{3}^{v}\right],\left[\mathbb{D}_{2}^{v}\right]\right\}, \\
{\left[\mathrm{O}(2)^{-}\right] \odot\left[\mathrm{O}(2)^{-}\right] } & =\left\{\left[\mathbb{Z}_{2}^{-}\right],\left[\mathrm{O}(2)^{-}\right]\right\} .
\end{aligned}
$$

Sketch of proof. We will only focus on the clips operation $\left[\mathrm{O}(2)^{-}\right] \odot\left[\mathbb{D}_{2 n}^{h}\right]$ and consider thus intersections

$$
\mathrm{O}(2)^{-} \cap\left(g \mathbb{D}_{2 n}^{h} g^{-1}\right), \quad g \in \mathrm{O}(3) .
$$

There are only two nontrivial cases to work on, whether characteristic axes match or not.

(1) If primary axes match, then by (B-4) and (B-6) we must consider intersections

$$
\mathbb{Z}^{\sigma_{v}} \cap \mathbb{Z}_{2 n}^{-}, \quad \mathrm{SO}(2) \cap \mathbb{Z}_{2}^{p_{j}}, \quad \mathbb{Z}^{\sigma_{b}} \cap \mathbb{Z}_{2}^{\sigma_{q_{j}}},
$$

which reduce to $\mathbb{D}_{n}^{v}$ (see decomposition (B-2)).

(2) Suppose, moreover, that $p_{0}=(O z)$, in which case $\operatorname{SO}(2) \cap \mathbb{Z}_{2}^{p_{j}}=\mathbb{Z}_{2}$.

(a) For $n$ odd, there exists some secondary axes $q_{k}$ in the $x y$-plane (see Lemma B.7) and thus $\mathbb{Z}^{\sigma_{b}} \cap \mathbb{Z}_{2}^{\sigma_{q_{j}}}$ reduces to $\mathbb{Z}_{2}^{\sigma_{q_{k}}}$. Moreover, $\mathbb{Z}^{\sigma_{v}} \cap \mathbb{Z}_{2 n}^{-}$ reduces to some $\mathbb{Z}_{2}^{\sigma_{v}}$ with $v$ perpendicular to $p_{0}$ and $q_{j}$ and the final result is a subgroup conjugate to $\mathbb{D}_{2}^{v}$.

(b) For $n$ even, we obtain $\mathbb{Z}_{2}$.

(3) If now the primary axis $a$ of $g \mathbb{D}_{2 n}^{h} g^{-1}$ is $(O x)$, and no other characteristic axes correspond to $(O z)$ nor $(O y)$, then intersections (B-7) reduce to $\mathbb{Z}_{2}^{\sigma_{a}} \cap$ $\left(g \mathbb{Z}_{2 n}^{-} g^{-1}\right)$, which is conjugate to $\mathbb{Z}_{2}^{-}$.

\section{References}

[Auffray et al. 2015] N. Auffray, J. Dirrenberger, and G. Rosi, "A complete description of bidimensional anisotropic strain-gradient elasticity", Internat. J. Solids Structures 69-70 (2015), 195206.

[Backus 1970] G. Backus, "A geometrical picture of anisotropic elastic tensors", Rev. Geophys. 8:3 (1970), 633-671.

[Bredon 1972] G. E. Bredon, Introduction to compact transformation groups, Pure and Applied Mathematics 46, Academic, New York, 1972.

[Chossat and Guyard 1994] P. Chossat and F. Guyard, "A classification of 2-modes interactions with SO(3) symmetry and applications", pp. 79-95 in Dynamics, bifurcation and symmetry (Cargèse, France, 1993), edited by P. Chossat, NATO Adv. Sci. Inst. Ser. C Math. Phys. Sci. 437, Kluwer, Dordrecht, 1994. 
[Chossat and Guyard 1996] P. Chossat and F. Guyard, "Heteroclinic cycles in bifurcation problems with O(3) symmetry and the spherical Bénard problem”, J. Nonlinear Sci. 6:3 (1996), 201-238.

[Chossat et al. 1990] P. Chossat, R. Lauterbach, and I. Melbourne, "Steady-state bifurcation with O(3)-symmetry”, Arch. Rational Mech. Anal. 113:4 (1990), 313-376.

[Cosserat and Cosserat 1909] E. Cosserat and F. Cosserat, Théorie des corps déformables, Hermann, Paris, 1909.

[Curie 1894] P. Curie, "Sur la symétrie dans les phénomènes physiques, symétrie d'un champ électrique et d'un champ magnétique", J. Phys. Theor. Appl. 3:1 (1894), 393-415.

[Eringen 1966] A. C. Eringen, "Linear theory of micropolar elasticity", J. Math. Mech. 15:6 (1966), 909-923.

[Fedorov 1968] F. I. Fedorov, Theory of elastic waves in crystals, Springer, 1968.

[Forest 2005] S. Forest, "Mechanics of Cosserat media: an introduction", notes, Ecole des Mines de Paris, 2005.

[Forest and Sievert 2006] S. Forest and R. Sievert, "Nonlinear microstrain theories", Internat. J. Solids Structures 43:24 (2006), 7224-7245.

[Forte and Vianello 1996] S. Forte and M. Vianello, "Symmetry classes for elasticity tensors", $J$. Elasticity 43:2 (1996), 81-108.

[Forte and Vianello 1997] S. Forte and M. Vianello, "Symmetry classes and harmonic decomposition for photoelasticity tensors", Internat. J. Engrg. Sci. 35:14 (1997), 1317-1326.

[Geymonat and Weller 2002] G. Geymonat and T. Weller, "Classes de symétrie des solides piézoélectriques”, C. R. Math. Acad. Sci. Paris 335:10 (2002), 847-852.

[Golubitsky et al. 1988] M. Golubitsky, I. Stewart, and D. G. Schaeffer, Singularities and groups in bifurcation theory, vol. II, Applied Mathematical Sciences 69, Springer, 1988.

[Gurtin 1973] M. E. Gurtin, "The linear theory of elasticity", pp. 1-295 in Mechanics of solids, vol. II: Linear theories of elasticity and thermoelasticity; linear and nonlinear theories of rods, plates, and shells, edited by C. Truesdell, Springer, 1973.

[Ihrig and Golubitsky 1984] E. Ihrig and M. Golubitsky, "Pattern selection with O(3) symmetry", Phys. D 13:1-2 (1984), 1-33.

[Jagodzinski 1955] H. Jagodzinski, "Kristallographie", pp. 1-103 in Handbuch der Physik, vol. VII/1, Springer, 1955.

[Le Quang and He 2011] H. Le Quang and Q.-C. He, "The number and types of all possible rotational symmetries for flexoelectric tensors", Proc. R. Soc. Lond. Ser. A Math. Phys. Eng. Sci. 467:2132 (2011), 2369-2386.

[Love 1944] A. E. H. Love, A treatise on the mathematical theory of elasticity, 4th ed., Dover, New York, 1944.

[Mann 1962] L. N. Mann, "Finite orbit structure on locally compact manifolds", Michigan Math. J. 9 (1962), 87-92.

[Michel 1980] L. Michel, "Symmetry defects and broken symmetry: configurations; hidden symmetry", Rev. Modern Phys. 52:3 (1980), 617-651.

[Mindlin 1964] R. D. Mindlin, "Micro-structure in linear elasticity", Arch. Rational Mech. Anal. 16 (1964), 51-78.

[Mindlin and Eshel 1968] R. D. Mindlin and N. N. Eshel, "On first strain-gradient theories in linear elasticity”, Internat. J. Solids Structures 4:1 (1968), 109-124.

[Mostow 1957] G. D. Mostow, "On a conjecture of Montgomery", Ann. of Math. (2) 65 (1957), 513-516. 
[Olive 2014] M. Olive, Géométrie des espaces de tenseurs: une approche effective appliquée à la mécanique des milieux continus, Ph.D. thesis, Aix-Marseille Université, 2014, Available at http:// www.theses.fr/2014AIXM4745.

[Olive and Auffray 2013] M. Olive and N. Auffray, "Symmetry classes for even-order tensors", Math. Mech. Complex Syst. 1:2 (2013), 177-210.

[Olive and Auffray 2014] M. Olive and N. Auffray, "Symmetry classes for odd-order tensors", ZAMM Z. Angew. Math. Mech. 94:5 (2014), 421-447.

[Portigal and Burstein 1968] D. L. Portigal and E. Burstein, "Acoustical activity and other first-order spatial dispersion effects in crystals”, Phys. Rev. 170:3 (1968), 673-678.

[Schouten 1951] J. A. Schouten, Tensor analysis for physicists, Oxford, 1951.

[Sternberg 1994] S. Sternberg, Group theory and physics, Cambridge University, 1994.

[Weller 2004] T. Weller, Etude des symétries et modèles de plaques en piézoélectricité linéarisée, Ph.D. thesis, Université Montpellier 2, 2004, Available at https://www.theses.fr/2004MON20036.

[Zou et al. 2013] W.-N. Zou, C.-X. Tang, and E. Pan, "Symmetry types of the piezoelectric tensor and their identification”, P. Roy. Soc. A 469:2155 (2013), 20120755.

Received 4 Jan 2019. Revised 16 Jun 2019. Accepted 2 Sep 2019.

MARC OLIVE: marc.olive@math.cnrs.fr

Laboratoire de Mécanique et Technologie, École Normale Supérieure Paris-Saclay,

Centre National de la Recherche Scientifique, Université Paris-Saclay, Cachan, France

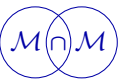





\title{
LOW-TEMPERATURE RATCHET CURRENT
}

\author{
JUSTINE LOUIS
}

We give an explicit expression for the low-temperature ratchet current in a multilevel system and find its numerical value as the number of states goes to infinity.

\section{Introduction}

In [Maes et al. 2014], the authors study stationary occupations in nonequilibrium multilevel systems at low temperatures. The system under consideration is an irreducible continuous time Markov jump process on a set of states $K$ with transition rates $\lambda(x, y)$ expressed in terms of the reactivities $a(x, y)$ and depending on the inverse temperature $\beta$. It is assumed that the system is in contact with an environment at uniform temperature and also subject to external forcing which breaks the Boltzmann occupation statistics. Being away from detailed balance implies that a current can flow in the system. To achieve nonequilibrium conditions, different physical models exist such as flashing ratchets. This corresponds to a random flipping between a flat potential and a nontrivial energy landscape. This is known as a continuous time Parrondo game [Parrondo 1998] and is studied in [Maes et al. 2014] as an example of their asymptotic formula for the stationary occupations. The authors model the multilevel system by a graph consisting of two rings of $N$ vertices which represent the states, and the edges stand for the preferred successors. This enables them to determine the direction of the ratchet current at low temperatures, which cannot be determined by entropic considerations only, and to evaluate it numerically while in the present paper we give an exact expression for it up to exponentially small corrections. This calculation confirms their result and in particular that the ratchet current is positive. The proof is based on an application of the Tutte matrix tree theorem. In the present section, we recall the definitions and results from [Maes et al. 2014, §3], and in the next section we give an explicit expression for the ratchet current and find its limit as the number of states goes to infinity.

\section{Communicated by Raffaele Esposito.}

The author acknowledges support from Swiss National Science Foundation grant 200021_132528/1. MSC2010: primary 05C30, 82C03; secondary 05C05, 05C20, 05C50.

PACS2010: 05.70.Ln.

Keywords: nonequilibrium statistical mechanics, direction current, Tutte matrix tree theorem. 
The zero-temperature logarithmic limit denoted by $\phi(x, y)$ is given by

$$
\phi(x, y):=\lim _{\beta \rightarrow \infty} \frac{1}{\beta} \log \lambda(x, y) .
$$

The zero-temperature logarithmic limit of the escape rates of state $x$ is denoted by $\Gamma(x)$ and given by

$$
\Gamma(x):=-\lim _{\beta \rightarrow \infty} \frac{1}{\beta} \log \left(\sum_{y} \lambda(x, y)\right)=-\max _{y} \phi(x, y) .
$$

The logarithmic-asymptotic transition probability is given by $e^{-\beta U(x, y)}$ where

$$
U(x, y):=-\Gamma(x)-\phi(x, y) .
$$

We have $U(x, y) \geqslant 0$ for all $x, y \in K$. The smaller $U(x, y)$ is, the larger is the probability of transition from state $x$ to state $y$. Hence, the set of preferred successors of $x$ is defined by

$$
\{y \in K \mid U(x, y)=0\} .
$$

When $U(x, y)=0$, the probability of transition from $x$ to $y$ is high. Thus, we consider the directed graph $K^{D}$ defined by the vertex set $K$ and edge set $\{(x, y) \mid$ $U(x, y)=0\}$ where $(x, y)$ indicates an oriented edge from $x$ to $y$. The transition rates are related to the reactivities by the relation

$$
\lambda(x, y)=a(x, y) e^{-\beta(\Gamma(x)+U(x, y))} .
$$

Using the Kirchhoff formula on $K^{D}$, the stationary occupation $\rho$ is given by

$$
\rho(x)=\frac{W(x)}{\mathscr{Z}}, \quad \text { where } W(x)=\sum_{\mathcal{T}_{x}} \prod_{(y, z) \in \mathscr{T}_{x}} \lambda(y, z) \text { and } \mathscr{E}=\sum_{x \in K} W(x)
$$

where the sum runs over all oriented edges $(y, z)$ in the in-tree $\mathscr{T}_{x}$. The lowtemperature asymptotic of the stationary occupation is given in:

Theorem [Maes et al. 2014, Theorem 2.1]. There is $\epsilon>0$ so that as $\beta \rightarrow \infty$,

with

$$
\rho(x)=\frac{1}{\mathscr{L}} A(x) e^{\beta(\Gamma(x)-\Theta(x))}\left(1+O\left(e^{-\beta \epsilon}\right)\right)
$$

$$
\begin{aligned}
& \Theta(x):=\min _{\mathcal{T}_{x}} U\left(\mathscr{T}_{x}\right) \quad \text { for } \quad U\left(\mathscr{T}_{x}\right):=\sum_{\left(y, y^{\prime}\right) \in \mathcal{T}_{x}} U\left(y, y^{\prime}\right), \\
& A(x):=\sum_{\mathcal{T}_{x} \in M(x)} \prod_{\left(y, y^{\prime}\right) \in \mathcal{T}_{x}} a\left(y, y^{\prime}\right)=e^{o(\beta)}
\end{aligned}
$$

where the last sum runs over all spanning trees minimizing $U\left(\mathscr{T}_{x}\right)$ (i.e., $\mathscr{T}_{x} \in M(x)$ if $\Theta(x)=U\left(\mathscr{T}_{x}\right)$ ). 


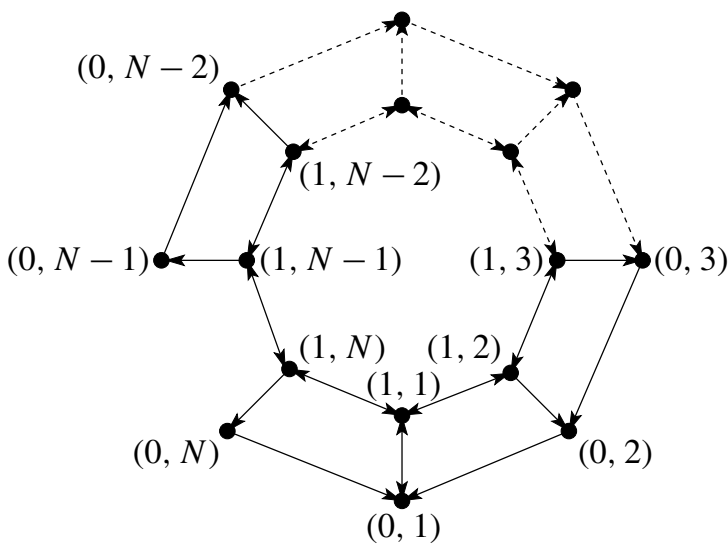

Figure 1. The directed graph $K^{D}$.

The case $a \gg 1$ satisfies detailed balance, and the model is then running on a single ring while in the case $a \ll 1$ the rings are uncoupled and detailed balance happens again for $a=0$. We thus consider the case $a=1$ corresponding to the nonequilibrium situation. The states, or energy levels, on the outer ring are denoted by $(0, i)$ and on the inner ring by $(1, i)$, where $i=1, \ldots, N$. The energies are denoted by $E_{i}, i=1, \ldots, N$, and are such that $E_{1}<\cdots<E_{N}$. The transition rates on the outer ring are thus given by

$$
\lambda((i, 0),(i+1,0))=e^{\beta\left(E_{i}-E_{i+1}\right) / 2}, \quad \lambda((i+1,0),(i, 0))=e^{\beta\left(E_{i+1}-E_{i}\right) / 2} .
$$

On the inner ring, the transition rates are constant and equal to one, that is,

$$
\lambda((i, 1),(i+1,1))=\lambda((i+1,1),(i, 1))=1 .
$$

The two rings are connected with transition rates constant and equal to one:

$$
\lambda((i, n),(i, 1-n))=1, \quad \text { where } n \in\{0,1\} .
$$

The digraph $K^{D}$ modeling the ratchet is represented in Figure 1. In the present case, for all $x \in K$, there exists an in-spanning tree $\mathscr{T}_{x}$ in $K^{D}$, so that $U\left(\mathscr{T}_{x}\right)=0$, and therefore, $\Theta(x)=0$. Let $\mathscr{D}$ be the set of states for which $\Gamma(x)=0$; it is given by $\mathscr{D}=\{(1,0),(i, 1), i=1, \ldots, N\}$. We denote $f \simeq g$ if $f=g+O\left(e^{-\beta \epsilon}\right)$ as $\beta \rightarrow \infty$. For $x \in \mathscr{D}$, we have $\rho(x) \simeq|M(x)| / \mathscr{L}$, where $|M(x)|$ is the number of in-spanning trees in $K^{D}$. For $x \notin \mathscr{D}$, the stationary distribution is exponentially small since from the theorem it is given by $\rho(x) \simeq|M(x)| e^{\beta \Gamma(x)} / \mathscr{W}$, with $\Gamma(x)<0$. The stationary ratchet current in the clockwise direction is given by

$$
J_{R}=j((i+1,0),(i, 0))+j((i+1,1),(i, 1)), \quad \text { for } i=1, \ldots, N,
$$

where $j(x, y)=\lambda(x, y) \rho(x)-\lambda(y, x) \rho(y)$. 
For $i=1$,

$$
J_{R}=j((2,0),(1,0))+j((2,1),(1,1))
$$

On the outer ring, $j((2,0),(1,0))=\lambda((2,0),(1,0)) \rho(2,0)-\lambda((1,0),(2,0)) \rho(1,0)$ with

$$
\begin{gathered}
\lambda((1,0),(2,0)) \simeq 0, \quad \lambda((2,0),(1,0))=e^{\left(E_{2}-E_{1}\right) \beta / 2}, \\
\rho(2,0) \simeq \frac{|M(2,0)|}{\mathscr{L}} e^{\beta \Gamma(2,0)}=\frac{|M(2,0)|}{\mathscr{L}} e^{-\left(E_{2}-E_{1}\right) \beta / 2},
\end{gathered}
$$

so that $j((2,0),(1,0)) \simeq|M(2,0)| / \mathscr{L}$.

On the inner ring, $j((2,1),(1,1))=\lambda((2,1),(1,1)) \rho(2,1)-\lambda((1,1),(2,1)) \rho(1,1)$ with

$$
\begin{gathered}
\lambda((2,1),(1,1))=\lambda((1,1),(2,1))=1, \\
\rho(2,1) \simeq \frac{|M(2,1)|}{\mathscr{Z}}, \quad \rho(1,1) \simeq \frac{|M(1,1)|}{\mathscr{Z}},
\end{gathered}
$$

so that $j((2,1),(1,1)) \simeq(|M(2,1)|-|M(1,1)|) / \mathscr{W}$. The ratchet current is thus given by

$$
J_{R} \simeq \frac{1}{\mathscr{E}}(|M(2,0)|+|M(2,1)|-|M(1,1)|) .
$$

Considering converging arborescences, the Laplacian matrix of a directed graph is defined by $L=D-A$ where $D$ is the diagonal out-degree matrix and $A=\left(A_{i j}\right)$ is the adjacency matrix such that $A_{i j}$ is the number of directed edges from $i$ to $j$. The rows and columns of $L$ are indexed by the vertices of the graph. Here, we index it first by the states on the outer ring and then the ones on the inner ring, that is $(1,0),(2,0), \ldots,(N, 0),(1,1),(2,1), \ldots,(N, 1)$. The Tutte matrix tree theorem [Aigner 2007] relates the number of spanning arborescences converging to $x$ in $K^{D}$ to the cofactors of the Laplacian $\operatorname{det} L_{x, y}$. Let $x \in K$. Then for all $y \in K$,

$$
|M(x)|=(-1)^{x+y} \operatorname{det} L_{x, y} .
$$

In particular, for $y=x$, we have $|M(x)|=\operatorname{det} L_{x}$. Therefore, we have

$$
J_{R} \simeq \frac{1}{\mathscr{L}}\left(\operatorname{det} L_{(2,1)}+\operatorname{det} L_{(2,0)}-\operatorname{det} L_{(1,1)}\right) .
$$

The Laplacian matrix is given by

$$
L=\left(\begin{array}{c|c}
F & G \\
\hline \mathrm{Id} & C
\end{array}\right)
$$


where $F$ is the $N \times N$ lower triangular matrix given by

$$
F=\left(\begin{array}{rrrrr}
1 & & & & \\
-1 & 1 & & & \\
& \ddots & \ddots & & \\
& & -1 & 1 & \\
-1 & & & 0 & 1
\end{array}\right),
$$

$G$ is the $N \times N$ matrix such that all coefficients are zero except $G_{(1,0),(1,1)}=-1$, the matrix Id is the $N \times N$ identity matrix, and $C$ is the circulant matrix

$$
C=\left(\begin{array}{rrrrr}
3 & -1 & & & -1 \\
-1 & 3 & \ddots & & \\
& \ddots & \ddots & \ddots & \\
& & \ddots & \ddots & -1 \\
-1 & & & -1 & 3
\end{array}\right) .
$$

\section{Calculation of the ratchet current}

From [Maes et al. 2014], the numerator of $J_{R}$ is given by

$$
\operatorname{det} L_{(2,1)}+\operatorname{det} L_{(2,0)}-\operatorname{det} L_{(1,1)}=\operatorname{det} B_{N-1}-2 \operatorname{det} B_{N-2}-2
$$

where $B_{N}$ is the $N \times N$ tridiagonal matrix with 3 on the diagonal and -1 on the two off-diagonals which satisfies the recurrence relation $\operatorname{det} B_{N}=3 \operatorname{det} B_{N-1}-$ $\operatorname{det} B_{N-2}$ with det $B_{1}=3$ and $\operatorname{det} B_{2}=8$. By solving the associated characteristic equation, we get

$$
\operatorname{det} B_{N}=\frac{5-3 \sqrt{5}}{10}\left(\frac{3-\sqrt{5}}{2}\right)^{N}+\frac{5+3 \sqrt{5}}{10}\left(\frac{3+\sqrt{5}}{2}\right)^{N} .
$$

The normalization factor is given by

$$
\mathscr{L}=\sum_{x \in K} \sum_{\mathscr{T}_{x}} \prod_{(y, z) \in \mathscr{T}_{x}} \lambda(y, z) \simeq \sum_{x \in \mathscr{D}}|M(x)|=\sum_{x \in \mathscr{D}} \operatorname{det} L_{x} .
$$

The sum is over the states in $\mathscr{D}$ since the contribution of the states which are not in $\mathscr{D}$ is exponentially damped. Therefore, we have

$$
\mathscr{Z} \simeq \operatorname{det} L_{(1,0)}+\sum_{i=1}^{N} \operatorname{det} L_{(i, 1)} .
$$

We have

$$
\operatorname{det} L_{(1,0)}=\operatorname{det} C \text {. }
$$


The circulant matrix $C$ has eigenvalues $\mu_{j}=3-2 \cos (2 \pi j / N), j=0,1, \ldots, N-1$ [Biggs 1993]. Hence,

$$
\operatorname{det} L_{(1,0)}=\prod_{j=0}^{N-1}(3-2 \cos (2 \pi j / N))=U_{N-1}^{2}(\sqrt{5} / 2)
$$

where $U_{N}$ is the $N$-th degree Chebyshev polynomial of the second kind. Thus,

$$
\operatorname{det} L_{(1,0)}=\left(\frac{3+\sqrt{5}}{2}\right)^{N}+\left(\frac{3-\sqrt{5}}{2}\right)^{N}-2 .
$$

From the Tutte matrix tree theorem, the cofactor $(-1)^{N+i} \operatorname{det} L_{(i, 1)}$ is equal to the number of converging arborescences to $(i, 1)$ and is equal to the cofactor of the Laplacian where row $(i, 1)$ and any column are removed. Since the only nonzero element of $G$ is in column indexed by $(1,1)$, we choose to remove that one, so that

$$
|M(i, 1)|=(-1)^{(N+i)+(N+1)} \operatorname{det} L_{(i, 1),(1,1)}=(-1)^{i+1} \operatorname{det} C_{(i, 1),(1,1)}
$$

since $F$ is lower triangular. On the other hand, by adding to the first column of $C$ all the other ones, we have

$$
\operatorname{det} C=\left|\begin{array}{rrrrr}
1 & -1 & & & -1 \\
1 & 3 & \ddots & & \\
& -1 & \ddots & \ddots & \\
& & \ddots & \ddots & -1 \\
1 & & & -1 & 3
\end{array}\right|=\sum_{i=1}^{N}(-1)^{i+1} \operatorname{det} C_{(i, 1),(1,1)}
$$

Putting (1), (2), (3), and (4) together, we have

$$
\mathscr{L} \simeq 2 \operatorname{det} C=2\left(\frac{3+\sqrt{5}}{2}\right)^{N}+2\left(\frac{3-\sqrt{5}}{2}\right)^{N}-4 .
$$

Up to exponentially small corrections $e^{-\beta \epsilon}$, the ratchet current is given for all $N$ by

$$
\begin{aligned}
J_{R} \simeq\left(\frac{5+3 \sqrt{5}}{10}\left(\frac{3+\sqrt{5}}{2}\right)^{N-1}+\frac{5-3 \sqrt{5}}{10}\left(\frac{3-\sqrt{5}}{2}\right)^{N-1}-\frac{5+3 \sqrt{5}}{5}\left(\frac{3+\sqrt{5}}{2}\right)^{N-2}\right. \\
\left.\quad-\frac{5-3 \sqrt{5}}{5}\left(\frac{3-\sqrt{5}}{2}\right)^{N-2}-2\right) /\left(2\left(\frac{3+\sqrt{5}}{2}\right)^{N}+2\left(\frac{3-\sqrt{5}}{2}\right)^{N}-4\right) .
\end{aligned}
$$

As a consequence, in the large system size limit the current saturates and has the limit

$$
\lim _{N \rightarrow \infty} J_{R} \simeq \frac{1}{2}-\frac{1}{\sqrt{5}}
$$




\section{Acknowledgements}

The author thanks Anders Karlsson for suggesting this problem to her. She also thanks the referee for useful comments.

\section{References}

[Aigner 2007] M. Aigner, A course in enumeration, Graduate Texts in Mathematics 238, Springer, 2007.

[Biggs 1993] N. Biggs, Algebraic graph theory, 2nd ed., Cambridge University, 1993.

[Maes et al. 2014] C. Maes, K. Netočný, and W. O. de Galway, "Low temperature behavior of nonequilibrium multilevel systems”, J. Phys. A Math. Theor. 47:3 (2014), 035002.

[Parrondo 1998] J. M. R. Parrondo, "Reversible ratchets as Brownian particles in an adiabatically changing periodic potential”, Phys. Rev. E 57:6 (1998), 7297-7300.

Received 8 Jul 2019. Revised 12 Aug 2019. Accepted 24 Sep 2019.

JUSTINE LOUIS: justine.louis@unige.ch

Section de Mathématiques, Université de Genève, Geneva, Switzerland

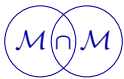





\title{
ESHELBY'S INCLUSION THEORY IN LIGHT OF NOETHER'S THEOREM
}

\author{
Salvatore Federico, Mawafag F. Alhasadi And Alfio Grillo
}

We dedicate this work to the memory of our maestro Professor Gaetano Giaquinta (Catania, Italy, 1945-2016), who first taught us Noether's theorem and showed us its unifying beauty.

In a variational setting describing the mechanics of a hyperelastic body with defects or inhomogeneities, we show how the application of Noether's theorem allows for obtaining the classical results by Eshelby. The framework is based on modern differential geometry. First, we present Eshelby's original derivation based on the cut-replace-weld thought experiment. Then, we show how Hamilton's standard variational procedure "with frozen coordinates", which Eshelby coupled with the evaluation of the gradient of the energy density, is shown to yield the strong form of Eshelby's problem. Finally, we demonstrate how Noether's theorem provides the weak form directly, thereby encompassing both procedures that Eshelby followed in his works. We also pursue a declaredly didactic intent, in that we attempt to provide a presentation that is as self-contained as possible, in a modern differential geometrical setting.

\section{Introduction}

In a classical paper, Eshelby [1951] introduced the concept of configurational force as the force required for a region containing a defect in a material body to undergo a material virtual displacement. This idea led to the mechanical Maxwell energymomentum tensor that has been subsequently termed Eshelby stress in continuum mechanics [Maugin and Trimarco 1992]. The procedure followed by Eshelby [1951] comprises a set of operations in which the elastic energy in the interior of a region and the net work that the surface tractions exert on the region are evaluated individually. In another work, Eshelby [1975] used Hamilton's standard variational approach of field theory and found his energy-momentum tensor directly, using the components of the regular spatial displacement and of the displacement gradient as the entities called fields in the jargon of field theory. In the same paper,

\section{Communicated by David J. Steigmann.}

PACS2010: 02.40.-k, 11.10.-z, 83.10.Ff, 02.30.Xx, 91.60.Ed.

Keywords: Eshelby stress, energy-momentum tensor, configurational mechanics, inclusion, defect,

Noether's theorem, variational principle. 
Eshelby [1975] also sketched the procedure for the case in which the fields are the components of the configuration map, which is the common choice in modern continuum mechanics.

Although initially conceived for a single inclusion or for a discrete set of inclusions, Eshelby's theory naturally applies to inhomogeneous materials or materials with continuous distributions of defects. Epstein and Maugin [1990] obtained the Eshelby stress using the concepts of material uniformity and material isomorphism introduced by Noll [1967] for inhomogeneous materials. Gurtin [1995; 2000] reformulated and generalized Eshelby's approach with the method of the varying control volumes and considered the Eshelby stress as the appropriate stress of an independent material balance law. The Eshelby stress has been seen as the object capturing inhomogeneities and singularities (e.g., [Epstein and Maugin 1990; Gurtin 1995; 2000; Epstein and Maugin 2000; Epstein and Elżanowski 2007; Verron et al. 2009; Weng and Wong 2009; Maugin 2011]), or the driving force of phenomena of material evolution such as plasticity and growth-remodeling (e.g., [Maugin and Epstein 1998; Epstein and Maugin 2000; Cermelli et al. 2001; Epstein 2002; Imatani and Maugin 2002; Grillo et al. 2003; 2005; Epstein 2009; 2015; Grillo et al. 2016; 2017; Hamedzadeh et al. 2019]), or phase transitions, or evolution of the interfaces among phases (e.g., [Gurtin 1986; 1993; Gurtin and Podio-Guidugli 1996; Fried and Gurtin 1994; 2004]).

In a didactic spirit, the aim of this work is to reproduce the results of Eshelby [1951; 1975] directly by means of the classical Noether theorem (for a translation into English of Noether's original 1918 paper, see [Noether 1971]) for continuum systems, as presented by Hill [1951]. The derivation is made using the components of the configuration map as the "fields" and those of the deformation gradient as the "gradients of the fields", while an appropriate "topological" transformation represents the material virtual displacement on the region containing the defect. We would like to emphasize that this work is more than a mere rewrite of Eshelby's findings in a more modern notation. While the relation between Eshelby's work and Noether's theorem has been highlighted in several papers (e.g., [Knowles and Sternberg 1971; Eshelby 1975; Fletcher 1976; Edelen 1981; Golebiewska Herrmann 1982; Olver 1984a; 1984b; Huang and Batra 1996; Kienzler and Herrmann 2000; Maugin 2011]), to the best of our knowledge, no work in the literature establishes an explicit relation between Eshelby's inclusion theory (and, specifically, the procedure to deal with the presence of the inclusion [Eshelby 1951; 1975]) and Noether's theorem.

In Section 2, we introduce the notation and give some basic definitions. In particular, we introduce standard and Eshelbian configurations and their variations, i.e., displacement fields. The setting is declaredly differential geometrical, although we avoid using differentiable manifolds for simplicity. In Section 3, we review, with 
our notation and within a suitable geometrical setting, Eshelby's original derivation [1951] of configurational forces. Similarly, in Section 4, we review Eshelby's variational derivation [1975]. Finally, in Section 5, which is the core of the work, we introduce Noether's theorem, and show how its application renders directly the results of both the previous derivations.

\section{Theoretical background}

In this section, we illustrate the notation that we employ and report some fundamental results relevant to this work. We generally use index-free notation, but sometimes it is useful to show the corresponding expression in index notation. Therefore, we present most expressions in both notations. In index notation, the customary Einstein summation convention for repeated indices is enforced throughout and a subscript preceded by a comma, as in $f_{, i}$, denotes partial differentiation with respect to its $i$-th argument.

2.1. General notation and basic definitions. Here we review some basic definitions of continuum mechanics, in order to elucidate the notation that we employ. The notation is essentially that of Truesdell and Noll [1965] and Marsden and Hughes [1983], with some modifications [Federico 2012; Federico et al. 2016]. We work in a simplified setting based on the use of affine spaces, whose rigorous definition can be found, e.g., in the treatise by Epstein [2010]. We could use a presentation in terms of differentiable manifolds [Noll 1967; Marsden and Hughes 1983; Epstein 2010; Segev 2013], but using affine spaces avoids many of the intricacies of higher-level differential geometry and makes the presentation more intuitive.

An affine space is a set $\mathcal{S}$, called the point space, considered together with a vector space $\mathcal{V}$, called the modeling space, and a mapping $\mathcal{S} \times \mathcal{S} \rightarrow \mathcal{V}:(x, y) \mapsto$ $y-x=\boldsymbol{u}$. This means that, at every point $x \in \mathcal{S}$, it is possible to univocally attach the vector given by $\boldsymbol{u}=y-x$, for every point $y \in \mathcal{S}$. The set of all vectors emanating from point $x$ is a vector space denoted $T_{x} \mathcal{S}=\{\boldsymbol{u} \in \mathcal{V}: \boldsymbol{u}=y-x$, for all $y \in \mathcal{S}\}$ and called tangent space to $\mathcal{S}$ at $x$. In the differential geometrical definition, the tangent space $T_{x} \mathcal{S}$ is the set of the vectors that are each tangent at $x$ to one of the infinite possible regular curves $c:[a, b] \rightarrow \mathcal{S}: s \mapsto c(s)$ such that $c\left(s_{0}\right)=x$, where $\left.s_{0} \in\right] a, b$, i.e., the vectors (see Figure 1)

$$
\boldsymbol{u}=\lim _{h \rightarrow 0} \frac{c\left(s_{0}+h\right)-c\left(s_{0}\right)}{h}=c^{\prime}\left(s_{0}\right) \in T_{x} \mathcal{S} .
$$

For the case of an affine space $\mathcal{S}$, this definition of tangent space $T_{x} \mathcal{S}$ coincides with that given by the expression $\boldsymbol{u}=y-x$. Indeed, by varying the curve passing by $x$, we obtain all possible "tip points" $y$ of the tangent vectors defined as $\boldsymbol{u}=y-x$. The dual space of $T_{x} \mathcal{S}$, i.e., the vector space of all linear maps $\varphi: T_{x} \mathcal{S} \rightarrow \mathbb{R}$, is 


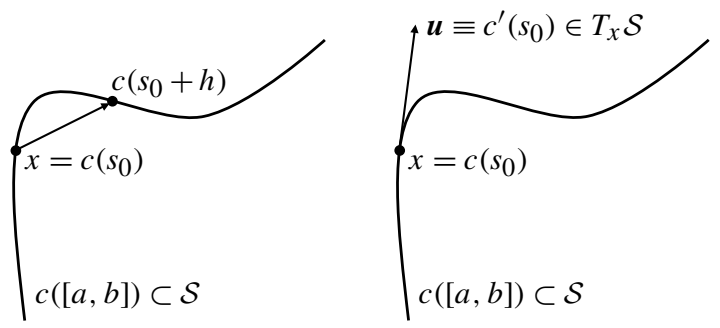

Figure 1. Differential geometrical definition of tangent vector at a point $x \in \mathcal{S}$. Left: the secant vector $c\left(s_{0}+h\right)-c\left(s_{0}\right)$ passing by $x=c\left(s_{0}\right)$. Right: the tangent vector $\boldsymbol{u}=c^{\prime}\left(s_{0}\right)$ at $x=c\left(s_{0}\right)$, obtained as the limit of the secant.

denoted $T_{x}^{\star} \mathcal{S}$ and is called the cotangent space to $\mathcal{S}$ at $x$. The disjoint unions of all tangent and cotangent spaces are called tangent bundle $T \mathcal{S}$ and cotangent bundle $T^{\star} \mathcal{S}$, respectively.

Vector fields and covector fields (or fields of one-forms) on an open set $\mathcal{A} \subseteq \mathcal{S}$ are maps

$$
\begin{aligned}
& \boldsymbol{u}: \mathcal{A} \subseteq \mathcal{S} \rightarrow T \mathcal{S}: x \mapsto \boldsymbol{u}(x) \in T_{x} \mathcal{S}, \\
& \boldsymbol{\varphi}: \mathcal{A} \subseteq \mathcal{S} \rightarrow T^{\star} \mathcal{S}: x \mapsto \boldsymbol{\varphi}(x) \in T_{x}^{\star} \mathcal{S},
\end{aligned}
$$

and tensor fields of higher order are defined analogously. Rather than speaking of contractions of vectors and covectors in a specific tangent and cotangent space, we can directly speak of the contractions of vector fields and covector fields in the tangent and cotangent bundle, and we denote the contraction by means of simple juxtaposition, i.e.,

$$
\boldsymbol{\varphi} \boldsymbol{u}=\boldsymbol{u} \boldsymbol{\varphi}=\varphi_{a} u^{a} .
$$

The physical space $\mathcal{S}$ is equipped with a metric tensor $\boldsymbol{g}$, a symmetric and positive definite second-order tensor field defining the scalar product of two vector fields as

$$
\boldsymbol{g}: T \mathcal{S} \times T \mathcal{S} \rightarrow \mathbb{R}:(\boldsymbol{u}, \boldsymbol{v}) \mapsto\langle\boldsymbol{u}, \boldsymbol{v}\rangle \equiv \boldsymbol{g}(\boldsymbol{u}, \boldsymbol{v})=u^{a} g_{a b} v^{b}
$$

We assume use of the Levi-Civita connection, i.e., the covariant derivative associated with the metric tensor $\boldsymbol{g}$ via the Christoffel symbols given by (see, e.g., [Marsden and Hughes 1983])

$$
\gamma_{b c}^{a}=\frac{1}{2} g^{a d}\left(g_{c d, b}+g_{b d, c}-g_{b c, d}\right),
$$

which are symmetric in their lower indices, i.e., $\gamma_{b c}^{a}=\gamma_{c b}^{a}$. The covariant derivative $\nabla_{\boldsymbol{u}} \boldsymbol{v}$ of the vector field $\boldsymbol{v}$ in the direction of the vector field $\boldsymbol{u}$ has the component 
expression

$$
\left[\nabla_{\boldsymbol{u}} \boldsymbol{v}\right]^{a} \equiv v_{\mid b}^{a} u^{b}=v_{, b}^{a} u^{b}+\gamma_{b c}^{a} v^{c} u^{b}
$$

and defines the gradient grad $\boldsymbol{v}$ as the tensor field such that its definition as a linear map is $(\operatorname{grad} \boldsymbol{v}) \boldsymbol{u} \equiv \nabla_{\boldsymbol{u}} \boldsymbol{v}$, with components $[\operatorname{grad} \boldsymbol{v}]_{b}^{a}=v^{a}{ }_{b}$. The covariant derivative and the gradient of a tensor field of arbitrary order are defined analogously.

Remark. A scalar is a tensor of order zero and thus we find it more natural to use the convention adopted by, e.g., Epstein [2010, p. 116] and to consider the gradient of a scalar field $f$ as the covector field (or one-form) grad $f$ such that $(\operatorname{grad} f)(\boldsymbol{u})=\nabla_{\boldsymbol{u}} f$, as for a tensor of any other order. Accordingly, the components of grad $f$ are $f_{, a}$. The other possible convention is that adopted by Marsden and Hughes [1983, p. 69], according to which the gradient of $f$ is the vector field with components $g^{a b} f_{, b}$. Note that, in either case, since $f$ is a tensor of order zero, the Christoffel symbols of the connection are not involved in the gradient, which is thus connection-independent. There are several advantages in defining the gradient as a covector. First, this definition is metric-independent, whereas the vector definition clearly necessitates that a metric tensor $\boldsymbol{g}$ be defined. Second, the covector definition accommodates the analytical mechanical definition of force as a covector field: indeed, an integrable force is the negative of the gradient of a potential energy and is thus consistently represented as a covector field. Finally, with the covector definition of grad $f$, we have the remarkable chain of identities

$$
\nabla f \equiv \operatorname{grad} f \equiv \mathrm{d} f \equiv \mathrm{D} f
$$

where $\mathrm{d} f$ is the exterior derivative of $f$, when seen as a zero-form (see, e.g., [Epstein 2010, p. 116]), and D $f$ is the Fréchet derivative (or tangent map) of $f$, when seen as a point map from $\mathcal{A} \subset \mathcal{S}$ into $\mathbb{R}$.

In the following, the physical space $\mathcal{S}$ is identified with the affine space $\mathbb{E}^{3}$, which is $\mathbb{R}^{3}$ considered both as the point space and as the modeling vector space.

2.2. Bodies, configurations, and the deformation gradient. In the simplified presentation that we adopt, a deformable continuous body $\mathcal{B}$ is identified with one of its placements in the physical space $\mathcal{S}$, and this particular placement is called reference configuration. The body is assumed to be endowed with the material metric $\boldsymbol{G}$, which induces the corresponding Levi-Civita connection, similarly to what was seen for the spatial metric $g$.

A configuration, or deformation, of the body is an embedding

$$
\phi: \mathcal{B} \rightarrow \mathcal{S}: X \mapsto x=\phi(X),
$$

i.e., a map such that its codomain-restriction $\phi: \mathcal{B} \rightarrow \phi(\mathcal{B})$ is a diffeomorphism, i.e., a continuous and differentiable map, which is invertible, with continuous and 


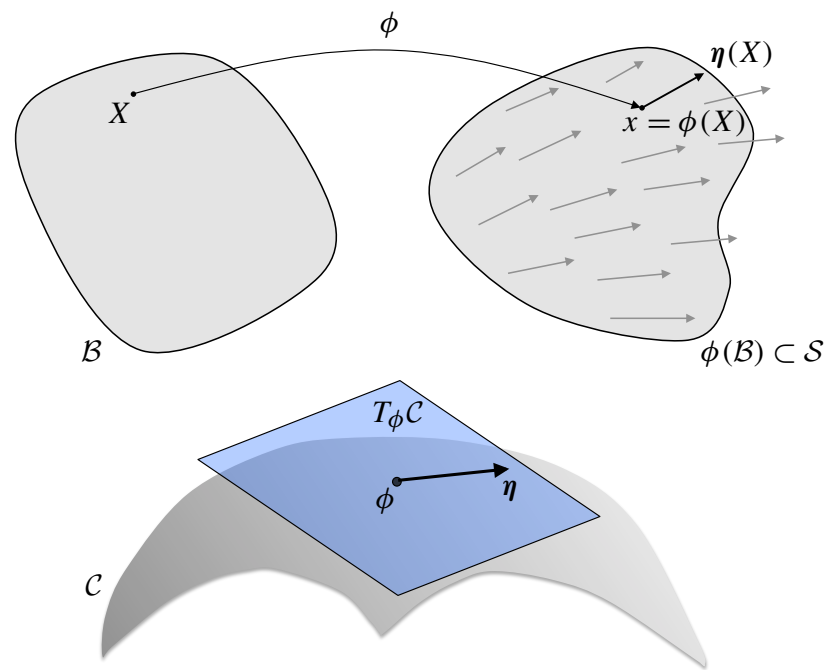

Figure 2. A conventional displacement field. Top: the displacement $\eta(X)=\eta(\Phi(x)) \in T_{x} \mathcal{S}$ as a tangent vector attached at $x=\phi(X)$. Bottom: the displacement field $\eta$ as a tangent vector attached at the configuration $\phi$, which is a point in the configuration space $\mathcal{C}$, here depicted as a surface, for the sake of an intuitive graphical representation.

differentiable inverse $\Phi \equiv \phi^{-1}: \phi(\mathcal{B}) \rightarrow \mathcal{B}$. The configuration $\phi$ maps material points $X=\left(X^{1}, X^{2}, X^{3}\right)$ in the body $\mathcal{B}$ into spatial points $x=\left(x^{1}, x^{2}, x^{3}\right)$ in $\mathcal{S}$, i.e., $\phi(X)=x$.

Since we are going to introduce another class of configurations, called Eshelbian, we shall refer to the standard definition of configuration given above as a conventional configuration. The set of all $k$-times differentiable conventional configuration maps (with $k \in \mathbb{N}$ ) constitutes the conventional configuration space $\mathcal{C}$ of the body $\mathcal{B}$. Since $\mathcal{S}$ is an affine space, the space $C^{k}(\mathcal{B}, \mathcal{S})$ of the $k$-times differentiable maps from $\mathcal{B}$ into $\mathcal{S}$ is an infinite-dimensional affine space. Thus, considering $\mathcal{C}$ as an open set in $C^{k}(\mathcal{B}, \mathcal{S})$ [Marsden and Hughes 1983] makes $\mathcal{C}$ an infinite-dimensional trivial manifold. A tangent vector $\boldsymbol{\eta}$ in the functional tangent space $T_{\phi} \mathcal{C}$ can be thought of as the tangent at $\phi$ to a curve of maps in $\mathcal{C}$ (i.e., a oneparameter family of maps in $\mathcal{C}$ ), and is a vector field covering the configuration $\phi$, i.e.,

$$
\eta: \mathcal{B} \rightarrow T \mathcal{S}: X \mapsto \eta(X) \in T_{\phi(X)} \mathcal{S}=T_{x} \mathcal{S} .
$$

The vector field $\boldsymbol{\eta}$ is called a (conventional) displacement field (and, when compatible with the constraints, but not necessarily attained by the body, it is called a virtual displacement). Figure 2 shows the displacement $\eta(X)=\eta(\Phi(x))$ as 
a tangent vector at $T_{x} \mathcal{S}$ and an illustration of the configuration space with the displacement field $\eta$ as a tangent vector at $T_{\phi} \mathcal{C}$.

The deformation gradient at point $X$ is the tangent map of $\phi$, i.e., the tensor

$$
(T \phi)(X)=\boldsymbol{F}(X): T_{X} \mathcal{B} \rightarrow T_{X} \mathcal{S},
$$

with $x=\phi(X)$, expressing the Fréchet derivative of $\phi$ at $X$. Since the existence of the Fréchet derivative of $\phi$ implies the existence of its Gâteaux derivative (or directional derivative), $\boldsymbol{F}(X)$ can be defined through the limit

$$
\left(\partial_{\boldsymbol{M}} \phi\right)(X):=\lim _{h \rightarrow 0} \frac{\phi(X+h \boldsymbol{M})-\phi(X)}{h}=[(T \phi)(X)] \boldsymbol{M}=[\boldsymbol{F}(X)] \boldsymbol{M},
$$

and the Gâteaux derivative $\partial_{M} \phi(X)$ of $\phi$ with respect to any tangent vector $\boldsymbol{M} \in$ $T_{X} \mathcal{B}$ equals the Fréchet derivative $\boldsymbol{F}(X) \boldsymbol{M}$, which is linear in $\boldsymbol{M}$. In components, (11) reads

$$
\left(\partial_{M} \phi\right)^{a}(X)=(T \phi)_{B}^{a}(X) M^{B}=F_{B}^{a}(X) M^{B}=\phi_{, B}^{a}(X) M^{B},
$$

where we recall that the comma denotes partial differentiation. Note that $\boldsymbol{F}(X)$ is a two-point tensor as it has the domain leg in $T_{X} \mathcal{B}$ and the codomain leg in $T_{X} \mathcal{S}$. As a tensor field, the deformation gradient is

$$
\boldsymbol{F}: \mathcal{B} \rightarrow T \mathcal{S} \otimes T^{\star} \mathcal{B}
$$

The deformation gradient $\boldsymbol{F}$ pushes-forward material vector fields $\boldsymbol{M}$ with components $M^{A}$ into spatial vector fields $\phi_{*} \boldsymbol{M}=(\boldsymbol{F} \circ \Phi)(\boldsymbol{M} \circ \Phi)$ with components $\left(F_{A}^{a} \circ \Phi\right)\left(M^{A} \circ \Phi\right)$. The inverse $\boldsymbol{F}^{-1}$ pulls-back spatial vector fields $\boldsymbol{m}$ with components $m^{a}$ into material vector fields $\phi^{*} \boldsymbol{m}=\left(\boldsymbol{F}^{-1} \circ \phi\right)(\boldsymbol{m} \circ \phi)$ with components $\left(\left(\boldsymbol{F}^{-1}\right)_{a}^{A} \circ \phi\right)\left(m^{a} \circ \phi\right)$. The transpose $\boldsymbol{F}^{T}$ pulls-back spatial covector fields $\pi$ with components $\pi_{a}$ into material covector fields $\phi^{*} \pi=\left(\boldsymbol{F}^{T} \circ \phi\right)(\boldsymbol{\pi} \circ \phi)$ with components $\left(\left(\boldsymbol{F}^{T}\right)_{A}^{a} \circ \phi\right)\left(\pi_{a} \circ \phi\right)=F_{A}^{a}\left(\pi_{a} \circ \phi\right)$. The inverse transpose $\boldsymbol{F}^{-T}$ pushes-forward material covector fields $\Pi$ with components $\Pi_{A}$ into spatial covector fields $\phi_{*} \boldsymbol{\Pi}=\left(\boldsymbol{F}^{-T} \circ \Phi\right)(\boldsymbol{\Pi} \circ \Phi)$ with components $\left(\left(\boldsymbol{F}^{-T}\right)_{a}^{A} \circ \Phi\right)\left(\Pi_{A} \circ \Phi\right)=$ $\left(\boldsymbol{F}^{-1}\right)_{a}^{A}\left(\Pi_{A} \circ \Phi\right)$.

The determinant $J=\operatorname{det} \boldsymbol{F}$ has the meaning of volume ratio, in the spirit of the theorem of the change of variables applied to the transformation from the spatial region $\phi(\mathcal{R}) \subset \mathcal{S}$ to the corresponding material region $\mathcal{R} \subset \mathcal{B}$.

2.3. Eshelbian configurations and their tangent maps. Grillo et al. [2003] introduced the concept of admissible reference configuration set of a body as the set of all reference configurations obtained by applying a diffeomorphism to the reference configuration $\mathcal{B}$ representing the body (which has some similarities with the idea of boundary reparametrizations introduced by Gurtin [1995]). Here, we make use of this concept in a slightly different way. 
An Eshelbian configuration $y$ is a diffeomorphism on the body $\mathcal{B}$. Since we define the body $\mathcal{B}$ as a trivial manifold, i.e., an open subset of the physical space $\mathcal{S}$, the codomain of an Eshelbian configuration $y$ should be the whole space $\mathcal{S}$ and the image would be an open set $\widetilde{\mathcal{B}}=y(\mathcal{B}) \subset \mathcal{S}$. However, if the body $\mathcal{B}$ were a nontrivial manifold, the image $\widetilde{\mathcal{B}}=y(\mathcal{B})$ would be another nontrivial manifold. To keep the notation as general as possible, we prefer to avoid declaring $\mathcal{S}$ as the codomain of $y$. Rather, we consider all admissible diffeomorphisms $y$, each with its image $\widetilde{\mathcal{B}}$, and we obtain the collection of all admissible reference configurations $\widetilde{\mathcal{B}}$, which clearly also contains $\mathcal{B}$ itself (see also [Grillo et al. 2003]). Then, we consider the union $\mathcal{N}=\bigcup_{y} \widetilde{\mathcal{B}}$ of these mutually diffeomorphic sets $\widetilde{\mathcal{B}}$, and define the generic Eshelbian configuration as

$$
y: \mathcal{B} \rightarrow \mathcal{N}: X \mapsto \tilde{X}=y(X)
$$

which has the further notational advantage of not tying $y$ to its specific image $\widetilde{\mathcal{B}}$.

Analogously to the case of a conventional configuration, the tangent map of an Eshelbian configuration at point $X$ is the tensor

$$
\left(T^{y}\right)(X): T_{X} \mathcal{B} \rightarrow T_{\widetilde{X}} \mathcal{N}
$$

with $\widetilde{X}=y(X)$. Again, $(T y)(X)$ is the Fréchet derivative of $y$ at $X$ and, since $y$ is a diffeomorphism, $(T y)(X)$ can be computed by means of the Gâteaux derivative of $y$ at $X$, i.e.,

$$
\left(\partial_{M} y\right)(X)=\lim _{h \rightarrow 0} \frac{y(X+h \boldsymbol{M})-y(X)}{h}=\left[\left(T^{y}\right)(X)\right] \boldsymbol{M} .
$$

The material identity map is the particular case of Eshelbian configuration obtained by considering that $\mathcal{B} \subset \mathcal{N}$, and is defined as

$$
X: \mathcal{B} \rightarrow \mathcal{B}: X \mapsto X=X(X),
$$

with the component representation

$$
X^{A}: \mathcal{B} \rightarrow \mathbb{R}: X \mapsto X^{A}=X^{A}(X) \equiv X^{A}\left(X^{1}, X^{2}, X^{3}\right) .
$$

Its tangent map is clearly the (material) identity tensor in $T \mathcal{B}$, i.e.,

$$
T X=I: T \mathcal{B} \rightarrow T \mathcal{B}, \quad(T \mathcal{X})_{B}^{A}=X_{, B}^{A}=\delta_{B}^{A} .
$$

Also in the case of Eshelbian configurations, we can exploit the affine structure of $\mathcal{S}$ : since all sets $\widetilde{\mathcal{B}}$ are open subsets of $\mathcal{S}$, also $\mathcal{N}=\bigcup_{y} \widetilde{\mathcal{B}} \subseteq \mathcal{S}$ is an open set, and thus we can define the space of all Eshelbian configurations as an open subset $\mathcal{M}$ of the infinite-dimensional affine space $C^{k}(\mathcal{B}, \mathcal{N})$, which makes $\mathcal{M}$ an infinite-dimensional trivial manifold. 
Remark. In our setting, in which the physical space $\mathcal{S}$ is an affine space and a body $\mathcal{B}$ is a subset of $\mathcal{S}$, the distinction between a conventional configuration $\phi: \mathcal{B} \rightarrow \mathcal{S}$ and an Eshelbian configuration $y: \mathcal{B} \rightarrow \mathcal{N}$ seems to fade out, because $\mathcal{N}=\bigcup_{y} \widetilde{\mathcal{B}} \subseteq \mathcal{S}$. However this is not the case, as will become clear from the explanation given in Section 3 (see also Figures 3 and 4). Moreover, when $\mathcal{B}$ is a general manifold, the distinction is fundamental. In this case, while a conventional configuration $\phi$ remains an embedding of $\mathcal{B}$ in $\mathcal{S}$, i.e., it gives $\mathcal{B}$ a placement $\phi(\mathcal{B}) \subset \mathcal{S}$, an Eshelbian configuration transforms the manifold $\mathcal{B}$ into a different manifold $\widetilde{\mathcal{B}}$.

A tangent vector $\boldsymbol{U} \in T_{x} \mathcal{M}$ is a vector field

$$
\boldsymbol{U}: \mathcal{B} \rightarrow T \mathcal{B}: X \mapsto \boldsymbol{U}(X) \in T_{X} \mathcal{B}
$$

and is called a material displacement field. When an Eshelbian configuration $y$ : $\mathcal{B} \rightarrow \mathcal{N}$ is defined as a perturbation of the material identity $X$, i.e.,

$$
\begin{gathered}
y(X)=X(X)+h \boldsymbol{U}(X) \quad=X+h \boldsymbol{U}(X), \\
y^{A}(X)=X^{A}(X)+h U^{A}(X)=X^{A}+h U^{A}(X),
\end{gathered}
$$

where $h \in \mathbb{R}$ is a smallness parameter and $\boldsymbol{U} \in T_{X} \mathcal{M}$, it is called an "infinitesimal transformation of the coordinates", in the language of field theory. Omitting the argument $X$, we can write

$$
y=x+h \boldsymbol{U}, \quad y^{A}=X^{A}+h U^{A} .
$$

The tangent map of $y$ in (22) is expressed by

$$
\begin{aligned}
T y & =T X+h \operatorname{Grad} \boldsymbol{U}=\boldsymbol{I}+h \mathrm{Grad} \boldsymbol{U}, \\
(T y)_{B}^{A} & =(T X)_{B}^{A}+h U_{\mid B}^{A}=\delta^{A}{ }_{B}+h U_{\mid B}^{A},
\end{aligned}
$$

where $\boldsymbol{I}$ is the material identity tensor and $\operatorname{Grad} \boldsymbol{U}$, with components $U_{\mid B}^{A}$, is the gradient (or covariant derivative) of $\boldsymbol{U}$. For $h \rightarrow 0$, the Jacobian determinant of $T y$ is

$$
\begin{aligned}
\operatorname{det}\left(T^{y}\right) & =\operatorname{det}(\boldsymbol{I}+h \operatorname{Grad} \boldsymbol{U})=1+h \operatorname{Tr}(\operatorname{Grad} \boldsymbol{U})+o(h) \\
& =1+h \operatorname{Div} \boldsymbol{U}+o(h)=1+h U_{\mid A}^{A}+o(h) .
\end{aligned}
$$

2.4. Conventions on forces and stresses. As mentioned in the remark on page 251, in the analytical mechanics/field theory approach, followed by, e.g., Hill [1951] and Eshelby [1975], forces are regarded as covector fields, acting on velocity or displacement vector fields. Thus, the contraction of a force with a velocity or displacement is given precisely by (3). Consequently, the first leg of the stress (the "force leg") is a covector, while the second leg (the "area leg") is a vector. Indeed, 
in the expression of Cauchy's theorem, the traction vectors relative to the spatial and material elements of area are given by

$$
\boldsymbol{t}_{\boldsymbol{n}}=\boldsymbol{\sigma} \boldsymbol{n}, \quad \mathfrak{t}_{N}=\boldsymbol{P} \boldsymbol{N}, \quad\left(\boldsymbol{t}_{\boldsymbol{n}}\right)_{a}=\sigma_{a}{ }^{b} n_{b}, \quad\left(\mathfrak{t}_{N}\right)_{a}=P_{a}{ }^{B} N_{B} .
$$

In (25), $\boldsymbol{n}$ is the normal covector to a surface element at the spatial point $x=$ $\phi(X)$ in the current configuration, $N$ is the normal covector to the corresponding surface element at the material point $X$ in the reference configuration, and the first Piola-Kirchhoff stress is related to Cauchy stress by means of the backward Piola transformation

$$
\boldsymbol{P}=J(\sigma \circ \phi) \boldsymbol{F}^{-T}, \quad P_{a}{ }^{B}=J\left(\sigma_{a}^{b} \circ \phi\right)\left(\boldsymbol{F}^{-T}\right)_{b}{ }^{B} .
$$

Equations (25) and (26) show that the tractions $\boldsymbol{t}_{\boldsymbol{n}}$ and $\mathfrak{t}_{N}$ are indeed covectors if the Cauchy stress $\boldsymbol{\sigma}$ and the first Piola-Kirchhoff stress $\boldsymbol{P}$, respectively, are treated as "mixed" tensors (we remark that $\mathfrak{t}_{\boldsymbol{N}} \neq \boldsymbol{t}_{\boldsymbol{n}}$, since $\boldsymbol{N}$ is related to $\boldsymbol{n}$ by the formula of the change of area, also known as Nanson's formula; see, e.g., [Bonet and Wood 2008]).

\section{Eshelby's original derivation of the weak form}

Eshelby [1951] derived the weak form of the expression of the configurational force balance by means of a thought experiment subdivided in several steps. This form is weak as it is an integral equation expressing a virtual work. We note that, in this section, we define the total energy $\mathcal{E}_{\mathcal{D}}$ in a region $\mathcal{D}$ of the body as a functional on the manifold $\mathcal{M}$, the Eshelbian configuration space.

Eshelby [1951] considered a body $\mathcal{B}$, subjected to constraints and external loads, and in whose interior is located a defect of any kind: a point defect, a dislocation, an inclusion, or even a region in which the material properties are inhomogeneous. To fix ideas, we follow Eshelby's graphical example with a point defect, as shown in Figure 3. The left panel in Figure 3 shows what Eshelby called the original body, in which a region $\mathcal{D}$ (highlighted in dark gray), bounded by the smooth material surface $\Sigma=\partial \mathcal{D}$, is selected such that the defect is contained in $\mathcal{D}$. The right panel in Figure 3 represents a replica of the original body, in which a different region $\widetilde{\mathcal{D}}$ (also highlighted in dark gray), bounded by the smooth material surface $\widetilde{\Sigma}=\partial \widetilde{\mathcal{D}}$, is selected so that the defect is contained in $\widetilde{\mathcal{D}}$ (see also [Kienzler and Herrmann 2000]). Since $\Sigma$ and $\widetilde{\Sigma}$ are both smooth, it is always possible to find an Eshelbian configuration $y$ transforming $\mathcal{D}$ into $\widetilde{\mathcal{D}}$, i.e., $y(\mathcal{D})=\widetilde{\mathcal{D}}$. Moreover, if $\Sigma$ and $\widetilde{\Sigma}$ are "close enough", then $\widetilde{\mathcal{D}}$ is obtainable from $\mathcal{D}$ through a perturbation of the form defined in (21), whose domain restriction to $\mathcal{D}$ is

$$
y: \mathcal{D} \rightarrow \mathcal{B}: X \mapsto y(X)=X(X)+h \boldsymbol{U}(X),
$$



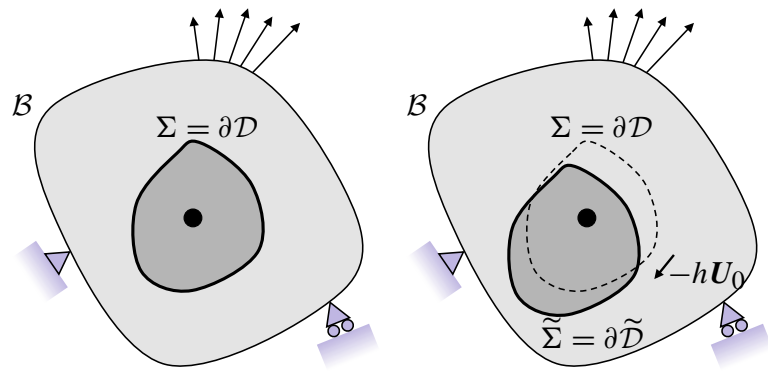

Figure 3. Determination of the force on a defect (the solid black circle). Left: original body, with the defect contained in a region $\mathcal{D}$, bounded by the smooth surface $\Sigma=\partial \mathcal{D}$. Right: replica body, with the defect contained in a different region $\widetilde{\mathcal{D}}$, bounded by the smooth surface $\widetilde{\Sigma}=\partial \widetilde{\mathcal{D}}$. As in Eshelby's original scheme [1975], here we depict the material displacement $h \boldsymbol{U}$ as being uniform over the material region $\mathcal{D}$ enclosed by the surface $\Sigma$, i.e., $h \boldsymbol{U}(X)=$ $-h \boldsymbol{U}_{0}$ for every $X \in \mathcal{D}$.

where we recall that $h$ is a smallness parameter. Note that Eshelby [1951] chose $h \boldsymbol{U}$ to be a uniform material displacement field $h \boldsymbol{U}(X)=-h \boldsymbol{U}_{0}$ over $\mathcal{D}$. Eshelby's choice makes the procedure easier to illustrate and yields directly the strong form of the inclusion problem. Here, we derive the weak form first and then obtain the strong form by adding Eshelby's assumption, $h \boldsymbol{U}(X)=-h \boldsymbol{U}_{0}$, at the very end. However, it is helpful to keep the uniform displacement $-h \boldsymbol{U}_{0}$ in one's mind and, to this end, we chose to represent this uniform displacement in Figure 3, following Eshelby's original thought experiment.

We remark that, since the map $y$ of (27) is Eshelbian, the body is undergoing no deformation, in the sense that it is not changing its shape, but only its configuration. Indeed, one chooses the surface $\Sigma$ enclosing the region $\mathcal{D}$ and the surface $\widetilde{\Sigma}$ enclosing the region $\widetilde{\mathcal{D}}$ independently and then finds a suitable $y$ mapping $\mathcal{D}$ into $\widetilde{\mathcal{D}}$. Clearly, this mere fact does not displace the defect at all, but simply represents a different choice of enclosing surface. The displacement of the defect in the reference configuration actually takes place when we replace the region $\mathcal{D}$ in the original body with the region $\widetilde{\mathcal{D}}$ cut from the replica body (which is straightforward in the case of an Eshelby rigid displacement $-h \boldsymbol{U}_{0}$ ), where $\mathcal{D}$ and $\widetilde{\mathcal{D}}$ are related by the material transformation $y$ described by (27). Note that, in this replacement, the defect is moved together with the region $\widetilde{\mathcal{D}}$ (see point (iii) below).

Our goal is to determine the variation in energy accompanying this change in reference configuration. In order to achieve this, we perform the thought experiment proposed by Eshelby $[1951 ; 1975]$ and described below. 
(i) In the original body, cut out the material in the region $\mathcal{D}$. If the body is prestressed for any reason, then apply traction forces to the boundary $\Sigma=\partial \mathcal{D}$ of the cavity that has been created, in order to avoid relaxation.

(ii) Similarly, in the replica body, cut out the material in the region $\widetilde{\mathcal{D}}=y(\mathcal{D})$ and apply suitable tractions to the boundary $\widetilde{\Sigma}=\partial \widetilde{\mathcal{D}}=\partial[y(\mathcal{D})] \equiv y(\partial \mathcal{D})$ to prevent relaxation. Let us denote the total elastic energy $\mathcal{E}_{\mathcal{D}}^{\mathrm{el}}: \mathcal{M} \rightarrow \mathbb{R}$ in $y(\mathcal{D})$ by

$$
\mathcal{E}_{\mathcal{D}}^{\mathrm{el}}(y)=\int_{y(\mathcal{D})} W=\int_{\mathcal{D}} \operatorname{det}(T y) W \circ y
$$

where we used the theorem of the change of variables to transform the integral over the displaced region $y(\mathcal{D})$ into an integral over the original region $\mathcal{D}$. Similarly, in the original region, the total elastic energy would be

$$
\mathcal{E}_{\mathcal{D}}^{\mathrm{el}}(X)=\int_{\mathcal{D}} W=\int_{\mathcal{D}} W \circ X,
$$

where we exploited the identity $X(X)=X$ in writing $W=W \circ X$. Therefore, the difference in energy due to the perturbation $y$ (i.e., due to the different selection of the surfaces $\widetilde{\Sigma}$ and $\Sigma$ ) is

$$
\mathcal{E}_{\mathcal{D}}^{\mathrm{el}}(y)-\mathcal{E}_{\mathcal{D}}^{\mathrm{el}}(X)=\int_{\mathcal{D}} \operatorname{det}(T y) W \circ y-\int_{\mathcal{D}} W \circ X=\int_{\mathcal{D}}[\operatorname{det}(T y) W \circ y-W \circ X] .
$$

By expressing the map $y$ as $y=X+h \boldsymbol{U}$ (see (21)), considering that, for $h \rightarrow 0$, $\operatorname{det} T \boldsymbol{y}=1+h \operatorname{Div} \boldsymbol{U}+o(h)($ see (24)) and

$$
W \circ y=W \circ(X+h \boldsymbol{U})=W \circ X+h[(\operatorname{Grad} W) \circ X] \boldsymbol{U}+o(h),
$$

(30) becomes

$$
\mathcal{E}_{\mathcal{D}}^{\mathrm{el}}(X+h \boldsymbol{U})-\mathcal{E}_{\mathcal{D}}^{\mathrm{el}}(X)=\int_{\mathcal{D}}[h(W \circ X) \operatorname{Div} \boldsymbol{U}+h[(\operatorname{Grad} W) \circ X] \boldsymbol{U}+o(h)] .
$$

Now, we can divide both sides of (32) by $h$ and take the limit for $h \rightarrow 0$ so that, on the left-hand side, we have the variational Gâteaux derivative of $\mathcal{E}_{\mathcal{D}}^{\mathrm{el}}$ with respect to the material displacement field $\boldsymbol{U} \in T_{\mathcal{X}} \mathcal{M}$, evaluated at the identity map $\mathcal{X}$, i.e.,

$$
\left(\partial_{\boldsymbol{U}} \mathcal{E}_{\mathcal{D}}^{\mathrm{el}}\right)(X)=\lim _{h \rightarrow 0} \frac{\mathcal{E}_{\mathcal{D}}^{\mathrm{el}}(X+h \boldsymbol{U})-\mathcal{E}_{\mathcal{D}}^{\mathrm{el}}(X)}{h}=\int_{\mathcal{D}}[(W \circ X) \operatorname{Div} \boldsymbol{U}+[(\operatorname{Grad} W) \circ X] \boldsymbol{U}] .
$$

By using the identities $(\operatorname{Grad} W) \circ \mathcal{X}=\operatorname{Grad} W$ and $W \circ X=W$, we can write

$$
\left(\partial_{\boldsymbol{U}} \mathcal{E}_{\mathcal{D}}^{\mathrm{el}}\right)(X)=\int_{\mathcal{D}}[W \operatorname{Div} \boldsymbol{U}+[\operatorname{Grad} W] \boldsymbol{U}]
$$



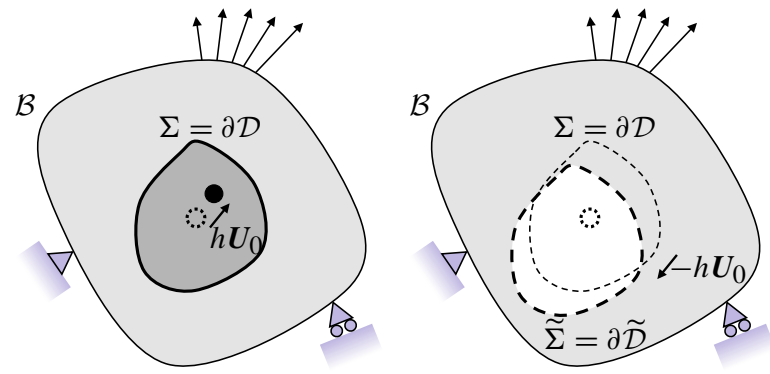

Figure 4. Before the deformation $\phi$ takes place, the region $\widetilde{\mathcal{D}}=$ $y(\mathcal{D})$ could be transplanted from the replica (right panel) to the original body (left panel), into the cavity resulting from the removal of the original region $\mathcal{D}$, by simply applying the negative of the displacement $-h \boldsymbol{U}_{0}$. This procedure effectively displaces the defect by the amount $h \boldsymbol{U}_{0}$ in the original body. We remark that this no longer holds after deformation has taken place.

which, by using Leibniz's rule and the identity $\operatorname{Div}(W \boldsymbol{U})=\operatorname{Div}(W \boldsymbol{I} \boldsymbol{U})$ (where $\boldsymbol{I}$ is the material identity tensor), becomes

$$
\left(\partial_{\boldsymbol{U}} \mathcal{E}_{\mathcal{D}}^{\mathrm{el}}\right)(X)=\int_{\mathcal{D}} \operatorname{Div}[W \boldsymbol{I} \boldsymbol{U}]
$$

(iii) Before the deformation $\phi$ occurs, the region $\widetilde{\mathcal{D}}=y(\mathcal{D})$ that had been isolated from the replica body could be "transplanted"1 into the cavity (resulting from the elimination of the original region $\mathcal{D}$ ) in the original body by simply applying the opposite displacement field $-h \boldsymbol{U}$. In Eshelby's choice of a uniform displacement, this would be the rigid translation $h \boldsymbol{U}_{0}$, as shown in Figure 4 . This is as if the defect had been displaced of the amount $h \boldsymbol{U}_{0}$.

However, after the deformation $\phi$ occurs, $\phi(\widetilde{\mathcal{D}})=\phi(y(\mathcal{D}))$ from the replica and $\phi(\mathcal{D})$ from the original body are different in general, and thus $\phi(\widetilde{\mathcal{D}})=\phi(\mathrm{Y}(\mathcal{D}))$ may not fit the cavity with deformed surface $\partial[\phi(\mathcal{D})] \equiv \phi(\partial \mathcal{D})=\phi(\Sigma)$ in the original body. Indeed, the points of the deformed surface $\partial[\phi(\mathcal{D})] \equiv \phi(\partial \mathcal{D})=\phi(\Sigma)$ in the original body and the points of the deformed surface $\partial[\phi(y(\mathcal{D}))] \equiv \phi(\partial(y(\mathcal{D})))=$ $\phi(\partial \widetilde{\mathcal{D}})=\phi(\widetilde{\Sigma})$ in the replica body generally differ by the (conventional spatial) displacement

$$
\phi(X+h \boldsymbol{U}(X))-\phi(X)=[\boldsymbol{F}(X)](h \boldsymbol{U}(X))+o(h),
$$

${ }^{1}$ We are borrowing the term "transplant" from Epstein and Maugin [2000] and Imatani and Maugin [2002], but with a more strictly "surgical" meaning. 
which, recalling that $X+h \boldsymbol{U}(X)=y(X)$ and $X=X(X)$, omitting the argument $X$ and using the linearity of $\boldsymbol{F}$, can be written as

$$
\phi \circ y-\phi \circ X=h \boldsymbol{F} \boldsymbol{U}+o(h) .
$$

In order to deform the surface $\phi(\Sigma)=\phi(\partial \mathcal{D})$ of the cavity in the original body in such a way that $\phi(\widetilde{\mathcal{D}})$ from the replica body can exactly fit in it, we must adjust the deformation. This can be achieved, in fact, by introducing a new deformation, $\bar{\phi}$, which, applied to $y(\mathcal{D})=\widetilde{\mathcal{D}}$, is such that the overall displacement is null, i.e.,

$$
\bar{\phi}(y(X))-\phi(X)=\mathbf{0} .
$$

Since $\bar{\phi}$ has to adjust $\phi$ in order to eliminate the mismatch generated by the combined effect of $y$ and $\phi$ (note how the composition $\phi \circ y$ is, in fact, the mathematical representation of the "combined effect"), it is natural to define $\bar{\phi}$ as a perturbation of $\phi$. Hence, we set

$$
\bar{\phi}=\phi+h \eta,
$$

where, without loss of generality, the same smallness parameter, $h$, is used as that defining $y=X+h \boldsymbol{U}$. With the aid of (39), and in the limit $h \rightarrow 0$, (38) becomes

$$
\begin{aligned}
\phi \circ(X+h \boldsymbol{U})+h \boldsymbol{\eta} \circ(X+h \boldsymbol{U}) & -\phi \circ X \\
& =h \boldsymbol{F} \boldsymbol{U}+o(h)+h \boldsymbol{\eta}+h^{2}[\boldsymbol{\eta} \circ X] \boldsymbol{U}+o\left(h^{2}\right) \\
& =h[\boldsymbol{F} \boldsymbol{U}+\boldsymbol{\eta}]+o(h)=\mathbf{0} .
\end{aligned}
$$

At the lowest order, (40) gives the condition sought for $\eta$, i.e., that it has to compensate for $\boldsymbol{U}$, thereby yielding

$$
\boldsymbol{F} \boldsymbol{U}+\boldsymbol{\eta}=\mathbf{0} \quad \Longrightarrow \quad-h \boldsymbol{\eta}=h \boldsymbol{F} \boldsymbol{U} .
$$

This interpretation of the displacement $\boldsymbol{\eta}$ is the core of Noether's theorem, which will be addressed in Section 5 .

The work necessary to adjust the deformation of $\mathcal{B} \backslash \mathcal{D}$ according to (39) is exerted by the first Piola-Kirchhoff surface traction $\boldsymbol{P}(-\boldsymbol{N})=-\boldsymbol{P} \boldsymbol{N}$, where the minus sign comes from the fact that we regard $N$ as the outward normal to the boundary $\Sigma=\partial \mathcal{D}$ of $\mathcal{D}$, which is inward with respect to the remainder $\mathcal{B} \backslash \mathcal{D}$ of the body. The integral of this work per unit referential area over the surface $\Sigma=\partial \mathcal{D}$ gives what Cermelli et al. [2001] called the "net work"

$$
\begin{aligned}
\mathcal{E}_{\mathcal{D}}^{\mathrm{nw}}(\mathrm{y}) & =\int_{\partial \mathcal{D}}(-\boldsymbol{P} \boldsymbol{N})(-h \boldsymbol{\eta})+o(h) \\
& =-h \int_{\partial \mathcal{D}}(\boldsymbol{P} \boldsymbol{N})(\boldsymbol{F} \boldsymbol{U})+o(h)=-h \int_{\partial \mathcal{D}}\left[\left(\boldsymbol{F}^{T} \boldsymbol{P}\right)^{T} \boldsymbol{U}\right] \boldsymbol{N}+o(h),
\end{aligned}
$$


where we rewrote the covector-vector contraction $(\boldsymbol{F} \boldsymbol{U})(\boldsymbol{P} N)$ by using the definition of transpose, i.e.,

$$
\begin{aligned}
(\boldsymbol{F} \boldsymbol{U})(\boldsymbol{P} N) & =F_{A}^{a} U^{A} P_{a}{ }^{B} N_{B}=\left(\boldsymbol{P}^{T}\right){ }_{a}^{B} F_{A}^{a} U^{A} N_{B}=\left[\left(\boldsymbol{F}^{T} \boldsymbol{P}\right)^{T}\right]_{A}^{B} U^{A} N_{B} \\
& =\left[\left(\boldsymbol{F}^{T} \boldsymbol{P}\right)^{T} \boldsymbol{U}\right] \boldsymbol{N} .
\end{aligned}
$$

Note that, for the sake of a lighter notation, we are writing $\boldsymbol{F}^{T}$ and $\boldsymbol{P}^{T}$ for $\boldsymbol{F}^{T} \circ \phi$ and $\boldsymbol{P}^{T} \circ \phi$. Rigorously speaking, the composition by $\phi$ would be necessary, since $\boldsymbol{F}^{T}$ and $\boldsymbol{P}^{T}$ are defined in the current configuration $\phi(\mathcal{B})$ [Marsden and Hughes 1983]. Since $N$ is the outward normal to $\Sigma=\partial \mathcal{D}$, the net work (42) is the negative of the work that the Piola tractions $\boldsymbol{P} \boldsymbol{N}$ would exert over the displacement $-h \boldsymbol{\eta}$ of (41) on the referential surface $\Sigma=\partial \mathcal{D}$, seen as the boundary of the referential region $\mathcal{D}$. This observation allows us to apply the divergence theorem to (42), which yields

$$
\mathcal{E}_{\mathcal{D}}^{\mathrm{nw}}(y)=-h \int_{\mathcal{D}} \operatorname{Div}\left[\left(\boldsymbol{F}^{T} \boldsymbol{P}\right)^{T} \boldsymbol{U}\right]+o(h) .
$$

This can be made into an increment by expressing the map $y$ as $y=X+h \boldsymbol{U}$, and considering that $\mathcal{E}_{\mathcal{D}}^{\mathrm{nw}}(X)=0$, i.e.,

$$
\mathcal{E}_{\mathcal{D}}^{\mathrm{nw}}(X+h \boldsymbol{U})-\mathcal{E}_{\mathcal{D}}^{\mathrm{nw}}(\mathcal{X})=-h \int_{\mathcal{D}} \operatorname{Div}\left[\left(\boldsymbol{F}^{T} \boldsymbol{P}\right)^{T} \boldsymbol{U}\right]+o(h) .
$$

Now, dividing by $h$ and passing to the limit $h \rightarrow 0$, we obtain the functional directional derivative

$$
\left(\partial_{\boldsymbol{U}} \mathcal{E}_{\mathcal{D}}^{\mathrm{nw}}\right)(X)=\lim _{h \rightarrow 0} \frac{\mathcal{E}_{\mathcal{D}}^{\mathrm{nw}}(X+h \boldsymbol{U})-\mathcal{E}_{\mathcal{D}}^{\mathrm{nw}}(X)}{h}=-\int_{\mathcal{D}} \operatorname{Div}\left[\left(\boldsymbol{F}^{T} \boldsymbol{P}\right)^{T} \boldsymbol{U}\right] .
$$

(iv) The deformed transformed region $\phi(\widetilde{\mathcal{D}})=\phi(y(\mathcal{D}))$ from the replica body can finally be exactly suited into the cavity left by the removal of $\mathcal{D}$ in the original body and we are able to weld together across the interface. We note that Eshelby [1975] needs to make considerations on the infinitesimals of order greater than $h$. In our approach, these are automatically taken care of (and eliminated) by the limit operation in (46). To cite Eshelby [1975] verbatim, except using our notation for the displacement,

"We are now left with the system as it was to begin with, except that the defect has been shifted by $-h \boldsymbol{U}=h \boldsymbol{U}_{0}$, as required."

The associated variation in the total energy $\varepsilon_{\mathcal{D}}: \mathcal{M} \rightarrow \mathbb{R}$ of the system is obtained as $\mathcal{E}_{\mathcal{D}}=\mathcal{E}_{\mathcal{D}}^{\mathrm{el}}+\mathcal{E}_{\mathcal{D}}^{\mathrm{nw}}$, i.e., by summing (35) and (46), i.e.,

$$
\left(\partial_{\boldsymbol{U}} \mathcal{E}_{\mathcal{D}}\right)(X)=\int_{\mathcal{D}} \operatorname{Div}[W \boldsymbol{I} \boldsymbol{U}]-\int_{\mathcal{D}} \operatorname{Div}\left[\left(\boldsymbol{F}^{T} \boldsymbol{P}\right)^{T} \boldsymbol{U}\right],
$$


which can be written as

$$
\left(\partial_{\boldsymbol{U}} \mathcal{E}_{\mathcal{D}}\right)(X)=\int_{\mathcal{D}} \operatorname{Div}\left[\mathfrak{E}^{T} \boldsymbol{U}\right]=\int_{\partial \mathcal{D}}(\mathfrak{E} N) \boldsymbol{U} .
$$

Equation (48) quantifies the variation in energy necessary to obtain a new reference configuration in which the defect is displaced in direction $\boldsymbol{U}$ with respect to the original one. In the context of the theory of defects, Eshelby [1951] called the tensor $\mathfrak{E}$, with the expression

$$
\mathfrak{E}=W \boldsymbol{I}^{T}-\boldsymbol{F}^{T} \boldsymbol{P}, \quad \mathfrak{E}_{A}{ }^{B}=W \delta_{A}{ }^{B}-F_{A}^{a} P_{a}{ }^{B},
$$

the Maxwell tensor of elasticity and later [Eshelby 1975] the energy-momentum tensor, in analogy with Maxwell's terminology from field theory. This analogy will be completely clear in Section 4. Later, Maugin and Trimarco [1992] gave $\mathfrak{E}$ the name of Eshelby stress in his honor.

At the end of Eshelby's thought experiment, we have the expression in (48), which can be thought of as the virtual work exerted by the Eshelby tractions $\mathbb{E} N$ on the material displacement field $\boldsymbol{U}$ on the boundary $\partial \mathcal{D}$ of the region $\mathcal{D}$. Using Eshelby's assumption $\boldsymbol{U}(X)=-\boldsymbol{U}_{0}$ for every $X \in \mathcal{D}$, we can write (48) as

$$
\left(\partial_{-U_{0}} \mathcal{E}_{\mathcal{D}}\right)(X)=-\int_{\mathcal{D}}(\operatorname{Div} \mathfrak{E}) \boldsymbol{U}_{0}=-\int_{\partial \mathcal{D}}(\mathfrak{E} N) \boldsymbol{U}_{0} .
$$

In order to obtain (in our notation) equation (17) in the paper by Eshelby [1951], we use Cartesian coordinates, so that it is legitimate to rewrite the integral as

$$
\mathcal{F} \boldsymbol{U}_{0}=\left(\partial_{-\boldsymbol{U}_{0}} \varepsilon_{\mathcal{D}}\right)(X)=-\left(\int_{\mathcal{D}} \operatorname{Div} \mathfrak{E}\right) \boldsymbol{U}_{0}=-\left(\int_{\partial \mathcal{D}} \mathfrak{E} N\right) \boldsymbol{U}_{0},
$$

where $\mathcal{F}$ was defined by Eshelby as the total inhomogeneity force, producing work over the uniform virtual displacement $\boldsymbol{U}_{0}$. We remark that the total inhomogeneity force $\mathcal{F}$ can only be defined in the case of Cartesian coordinates, which is the only particular case in which integration of a vector field makes sense (see the warning at page 134 in the text by Marsden and Hughes [1983]).

\section{Eshelby's variational derivation of the strong form}

In his seminal paper, Eshelby [1975] used a variational approach and wrote the Euler-Lagrange equations for a generic system with a potential energy depending - in the language of classical field theory — on fields, "gradients" of fields, and coordinates. In this quite general framework, elasticity can be seen as a particular case. Here, we follow Eshelby's derivation [1975] step by step, using our notation and adding our comments. Then, we shall show how this specializes to the case of large- and small-deformation elasticity. The only difference with 
Eshelby's procedure is that, whenever we look at the variational problem as an elasticity problem, our fields are the components of the configuration map, rather than the components of the displacement. Note that, in contrast with Section 3, here we define the total energy $\mathcal{E}_{\mathcal{D}}$ in a region $\mathcal{D}$ of the body as a functional on the manifold $\mathcal{C}$, the conventional configuration space.

Let us assume a potential energy density $W$, defined per unit referential volume, given by

$$
W(X)=\widehat{W}(\phi(X), \boldsymbol{F}(X), X),
$$

where $\phi$ is a collection of scalar fields (in the case of continuum mechanics, the configuration map, with components $\left.\phi^{a}\right), \boldsymbol{F}$ is the collection of the gradients of the fields (in our case, the deformation gradient, with components $F_{A}^{a}=\phi_{{ }_{,}}^{a}$ ), and $X$ is the collection of the independent variables (in our case, the material coordinates $X^{A}$ ). Note that we distinguish between the scalar field $W$ (function of the coordinates $X^{A}$ ) and the associated constitutive function $\widehat{W}$ (function of the fields $\phi^{a}$, the gradients $F_{A}^{a}=\phi^{a}{ }_{A}$, and the coordinates $\left.X^{A}\right)$. By using the material identity map $X$ of (17) (such that $X=X(X)$, in components, $X^{A}=X^{A}(X)$ ), the potential energy can be rewritten in the form

$$
W(X)=\widehat{W}(\phi(X), \boldsymbol{F}(X), X(X))=[\widehat{W} \circ(\phi, \boldsymbol{F}, X)](X) .
$$

Thus, by dropping the argument $X$ on the far left and the far right sides, we have

$$
W=\widehat{W} \circ(\phi, F, X) .
$$

In order to find the Euler-Lagrange equations associated with $W=\widehat{W} \circ(\phi, \boldsymbol{F}, X)$, we need to consider the total energy $\mathcal{E}_{\mathcal{B}}: \mathcal{C} \rightarrow \mathbb{R}$ over the whole body $\mathcal{B}$, given by

$$
\varepsilon_{\mathcal{B}}(\phi)=\int_{\mathcal{B}} W=\int_{\mathcal{B}} \widehat{W} \circ(\phi, \boldsymbol{F}, X),
$$

and calculate its variation with respect to a conventional displacement $\boldsymbol{\eta}$, which is given by the Gâteaux derivative

$$
\begin{aligned}
\left(\partial_{\eta} \mathcal{E}_{\mathcal{B}}\right)(\phi) & =\lim _{h \rightarrow 0} \frac{\mathcal{E}_{\mathcal{B}}(\phi+h \boldsymbol{\eta})-\mathcal{E}_{\mathcal{B}}(\phi)}{h} \\
& =\lim _{h \rightarrow 0} \frac{1}{h} \int_{\mathcal{B}}[\widehat{W} \circ(\phi+h \boldsymbol{\eta}, \boldsymbol{F}+h \operatorname{Grad} \boldsymbol{\eta}, X)-\widehat{W} \circ(\phi, \boldsymbol{F}, X)],
\end{aligned}
$$

with $\eta$ chosen in a suitable subset of $T_{\phi} \mathcal{C} \cap C^{1}(\mathcal{B}, T \mathcal{S})$, as will be clarified later in this section. In the jargon of field theory, this is called a "variation on the fields, with frozen coordinates", i.e., we are going to calculate the integral on the fixed domain $\mathcal{B}$. The transformation on the configuration map $\phi$ (the "fields" $\phi^{a}$ ) is 
given by

$$
\begin{aligned}
\phi & \mapsto \bar{\phi}=\phi+h \eta, \\
\phi^{a} & \mapsto \bar{\phi}^{a}=\phi^{a}+h \eta^{a},
\end{aligned}
$$

and the transformation on the tangent map $T \phi=\boldsymbol{F}$ (the "gradients" $F_{A}^{a}=\phi_{, A}^{a}$ ) is

$$
\begin{gathered}
T \phi=\boldsymbol{F} \mapsto T \bar{\phi}=\overline{\boldsymbol{F}}=T(\phi+h \boldsymbol{\eta})=\boldsymbol{F}+h \operatorname{Grad} \boldsymbol{\eta}, \\
\phi_{, A}^{a}=F_{A}^{a} \mapsto \bar{F}_{A}^{a}=\bar{\phi}_{, A}^{a}=\phi_{, A}^{a}+h \eta_{\mid A}^{a}=F_{A}^{a}+h \eta_{\mid A}^{a},
\end{gathered}
$$

where Grad $\eta$, with components $\eta_{\mid A}^{a}$, is the covariant derivative of the displacement $\boldsymbol{\eta}$.

We follow the standard derivation by expanding the argument of the integral as $\widehat{W} \circ(\phi+h \boldsymbol{\eta}, \boldsymbol{F}+h \operatorname{Grad} \boldsymbol{\eta}, X)-\widehat{W} \circ(\phi, \boldsymbol{F}, X)$

$$
=\frac{\partial \widehat{W}}{\partial \phi^{a}} \circ(\phi, \boldsymbol{F}, \mathcal{X}) h \eta^{a}+\frac{\partial \widehat{W}}{\partial F_{A}^{a}} \circ(\phi, \boldsymbol{F}, \mathcal{X}) h \eta_{\mid A}^{a}+o(h),
$$

substituting in (56), and performing the limit, which results in

$$
\begin{aligned}
\left(\partial_{\eta} \mathcal{E}_{\mathcal{B}}\right)(\phi) & =\int_{\mathcal{B}}\left[\frac{\partial \widehat{W}}{\partial \phi^{a}} \circ(\phi, \boldsymbol{F}, \mathcal{X}) \eta^{a}+\frac{\partial \widehat{W}}{\partial F_{A}^{a}} \circ(\phi, \boldsymbol{F}, \mathcal{X}) \eta_{\mid A}^{a}\right] \\
& =\int_{\mathcal{B}}\left[-f_{a} \eta^{a}+P_{a}{ }^{A} \eta_{\mid A}^{a}\right]=\int_{\mathcal{B}}[-\boldsymbol{f} \boldsymbol{\eta}+\boldsymbol{P}: \operatorname{Grad} \boldsymbol{\eta}],
\end{aligned}
$$

where $\boldsymbol{f}$ and $\boldsymbol{P}$ are given by

$$
\begin{aligned}
f_{a} & =-\frac{\partial \widehat{W}}{\partial \phi^{a}} \circ(\phi, \boldsymbol{F}, X), & \boldsymbol{f} & =-\frac{\partial \widehat{W}}{\partial \phi} \circ(\phi, \boldsymbol{F}, X), \\
P_{a}{ }^{A} & =\frac{\partial \widehat{W}}{\partial F_{A}^{a}} \circ(\phi, \boldsymbol{F}, X), & \boldsymbol{P} & =\frac{\partial \widehat{W}}{\partial \boldsymbol{F}} \circ(\phi, \boldsymbol{F}, X) .
\end{aligned}
$$

In the case of elasticity in continuum mechanics, when the potential is given as the sum of an elastic potential and a potential of the external body forces, i.e.,

$$
\widehat{W} \circ(\phi, \boldsymbol{F}, X)=\widehat{W}_{\mathrm{el}} \circ(\boldsymbol{F}, X)+\widehat{W}_{\mathrm{ext}} \circ(\phi, X),
$$

the covector field $\boldsymbol{f}$ and the tensor field $\boldsymbol{P}$ take the meaning of external body force per unit volume and first Piola-Kirchhoff stress, respectively. Now, considering that

$$
\boldsymbol{P}: \operatorname{Grad} \boldsymbol{\eta}=P_{a}{ }^{A} \eta_{\mid A}^{a}=\left(P_{a}{ }^{A} \eta^{a}\right)_{\mid A}-P_{a \mid A}{ }^{A} \eta^{a}=\operatorname{Div}\left(\boldsymbol{P}^{T} \boldsymbol{\eta}\right)-(\operatorname{Div} \boldsymbol{P}) \boldsymbol{\eta},
$$


the variation becomes

$$
\begin{aligned}
\left(\partial_{\eta} \mathcal{E}_{\mathcal{B}}\right)(\phi) & =\int_{\mathcal{B}}[-\boldsymbol{f} \boldsymbol{\eta}+\operatorname{Div}(\boldsymbol{\eta} \boldsymbol{P})-(\operatorname{Div} \boldsymbol{P}) \boldsymbol{\eta}] \\
& =-\int_{\mathcal{B}}(\boldsymbol{f}+\operatorname{Div} \boldsymbol{P}) \boldsymbol{\eta}+\int_{\mathcal{B}} \operatorname{Div}(\boldsymbol{\eta} \boldsymbol{P})
\end{aligned}
$$

and, by applying Gauss's divergence theorem,

$$
\left(\partial_{\eta} \mathcal{E}_{\mathcal{B}}\right)(\phi)=-\int_{\mathcal{B}}(\boldsymbol{f}+\operatorname{Div} \boldsymbol{P}) \boldsymbol{\eta}+\int_{\partial \mathcal{B}}(\boldsymbol{P} \boldsymbol{N}) \boldsymbol{\eta},
$$

where $\boldsymbol{N}$ is the normal to the boundary $\partial \mathcal{B}$ and $(\boldsymbol{P} N) \eta=\eta(P N)$.

We now look for a configuration $\phi$ at which $\mathcal{E}_{\mathcal{B}}(\phi)$ is stationary. For this purpose, we impose the condition $\left(\partial_{\eta} \mathcal{E}_{\mathcal{B}}\right)(\phi)=0$, in which $\phi$ is unknown, and we study it under the restriction that $\boldsymbol{\eta}$ vanishes on $\partial \mathcal{B}$ [Hill 1951]. This choice annihilates the surface integral on the right-hand side of (65), so that the stationarity condition becomes

$$
\left(\partial_{\eta} \mathcal{E}_{\mathcal{B}}\right)(\phi)=-\int_{\mathcal{B}}(\boldsymbol{f}+\operatorname{Div} \boldsymbol{P}) \boldsymbol{\eta}=0, \quad \boldsymbol{\eta} \in \mathcal{V},
$$

where $\mathcal{V}:=\left\{\boldsymbol{\eta} \in T_{\phi} \mathcal{C} \cap C^{1}(\mathcal{B}, T \mathcal{S}): \eta(X)=\mathbf{0}\right.$ for all $\left.X \in \partial \mathcal{B}\right\}$. We require now that (66) be satisfied for all $\eta \in \mathcal{V}$, which leads to the Euler-Lagrange equations

$$
\boldsymbol{f}+\operatorname{Div} \boldsymbol{P}=\mathbf{0}, \quad f_{a}+P_{a \mid A}^{A}=0 .
$$

If the external body forces acting on $\mathcal{B}$ are only those given by $\boldsymbol{f}$, which admit the potential density $\widehat{W}_{\text {ext }} \circ(\phi, X),(67)$ represents, in continuum mechanics, the Lagrangian (static) equilibrium equations, i.e., spatial equations described in terms of the material coordinates. If $\phi$ is a solution to (67), and the boundary of $\mathcal{B}$ can be written as the disjoint union of a Dirichlet part and a Neumann part, i.e., $\partial \mathcal{B}=\partial_{D} \mathcal{B} \sqcup \partial_{N} \mathcal{B}$, then the variation $\left(\partial_{\eta} \mathcal{E}_{\mathcal{B}}\right)(\phi)$ in (65) becomes

$$
\left(\partial_{\eta} \mathcal{E}_{\mathcal{B}}\right)(\phi)=\int_{\partial \mathcal{B}}(\boldsymbol{P} \boldsymbol{N}) \boldsymbol{\eta}=\int_{\partial_{\mathcal{N}} \mathcal{B}}(\boldsymbol{P} \boldsymbol{N}) \boldsymbol{\eta},
$$

where the surface integral is restricted to the Neumann boundary, $\partial_{N} \mathcal{B}$, because the displacement $\eta$, although arbitrary, has to vanish on the Dirichlet boundary, $\partial_{D} \mathcal{B}$. In this case, the stationarity condition on $\mathcal{E}_{\mathcal{B}}$ requires the vanishing of the surface integral on the far right-hand side of (65). This can be obtained if $\partial_{N} \mathcal{B}$ is a set of null measure, or if no contact forces are applied onto $\partial_{N} \mathcal{B}$. On the contrary, when contact forces are present, the stationarity condition on $\mathcal{E}_{\mathcal{B}}$ must be corrected by requiring that $\left(\partial_{\eta} \mathcal{E}_{\mathcal{B}}\right)(\phi)$ be balanced by the work performed by the contact forces on $\eta$. This result follows from the extended Hamilton principle [dell'Isola and Placidi 2011]. 
If (65) is referred to a set $\mathcal{D} \subset \mathcal{B}$, and is evaluated for a configuration $\phi$ solving (67), the volume integral vanishes by virtue of the Euler-Lagrange equations, while internal contact forces are exchanged through $\partial \mathcal{D}$. In this case, $\boldsymbol{\eta}$ is not required to vanish on $\partial \mathcal{D}$, and the variational procedure leads to

$$
\left(\partial_{\eta} \mathcal{E}_{\mathcal{D}}\right)(\phi)=\int_{\partial \mathcal{D}}(\boldsymbol{P} \boldsymbol{N}) \eta
$$

thereby returning the virtual work exerted by the contact forces acting on $\partial \mathcal{D}$.

Let us now assume that $\phi$ satisfies the Euler-Lagrange equations (67), and let us take the material gradient Grad $W$ of the energy density $W$, i.e., the partial derivatives of $W$ with respect to $X^{B}$,

$$
\begin{aligned}
W_{, B} & =[\widehat{W} \circ(\phi, \boldsymbol{F}, X)]_{, B} \\
& =\left[\frac{\partial \widehat{W}}{\partial \phi^{a}} \circ(\phi, \boldsymbol{F}, X)\right] \phi_{, B}^{a}+\left[\frac{\partial \widehat{W}}{\partial F_{A}^{a}} \circ(\phi, \boldsymbol{F}, X)\right] F_{A \mid B}^{a}+\frac{\partial \widehat{W}}{\partial X^{B}} \circ(\phi, \boldsymbol{F}, X) \\
& =-f_{a} F_{B}^{a}+P_{a}{ }^{A} F_{A \mid B}^{a}+\frac{\partial \widehat{W}}{\partial X^{B}} \circ(\phi, \boldsymbol{F}, X)
\end{aligned}
$$

where we used the definitions of the components of the deformation gradient, $F_{A}^{a}=\phi^{a}{ }_{, A}$, of the body force and the first Piola-Kirchhoff stress, and $F_{A \mid B}^{a}$ are the components of the third-order two-point tensor Grad $\boldsymbol{F}$. The last term in (70) is usually called "explicit" gradient of the field $W$ and denoted $\left.\left(\partial W / \partial X^{B}\right)\right|_{\text {expl }}$ in the literature (e.g., [Eshelby 1975; Epstein and Maugin 1990]), whereas we regard it as the collection of the partial derivatives of the constitutive function $\widehat{W}$ with respect to $X^{B}$ (which, we recall, are the functions such that $X^{B}(X)=X^{B}$ ). The negative of the "explicit" gradient defines the material inhomogeneity force or configurational force

$$
\mathfrak{F}=-\frac{\partial \widehat{W}}{\partial X} \circ(\phi, \boldsymbol{F}, X), \quad \mathfrak{F}_{A}=-\frac{\partial \widehat{W}}{\partial X^{A}} \circ(\phi, \boldsymbol{F}, X) .
$$

Substituting the expressions of the Lagrangian force $\boldsymbol{f}$, the Piola-Kirchhoff stress $\boldsymbol{P}$, and the configurational force $\mathfrak{F}$ into (70), we obtain

$$
\operatorname{Grad} W=-\boldsymbol{F}^{T} \boldsymbol{f}+\boldsymbol{P}: \operatorname{Grad} \boldsymbol{F}-\mathfrak{F},
$$

where the double contraction ": " in the second term is of the two legs of $\boldsymbol{P}$ with the first two legs of Grad $\boldsymbol{F}$. By invoking the symmetry of the Christoffel symbols $\Gamma_{B C}^{A}$ associated with the Levi-Civita connection induced by the material metric $\boldsymbol{G}$, so that $F_{A \mid B}^{a}=F_{B \mid A}^{a}$, we work out the second term on the right-hand side of (72) in components, i.e.,

$$
P_{a}{ }^{A} F_{A \mid B}^{a}=P_{a}{ }^{A} F_{B \mid A}^{a}=\left(P_{a}{ }^{A} F_{B}^{a}\right)_{\mid A}-P_{a \mid A}{ }^{A} F_{B}^{a},
$$


which, in component-free notation, reads

$$
\boldsymbol{P}: \operatorname{Grad} \boldsymbol{F}=\operatorname{Div}\left(\boldsymbol{F}^{T} \boldsymbol{P}\right)-\boldsymbol{F}^{T} \operatorname{Div} \boldsymbol{P}
$$

By substituting this result into (72), we obtain

$$
\begin{aligned}
\operatorname{Grad} W & =-\boldsymbol{F}^{T} \boldsymbol{f}+\operatorname{Div}\left(\boldsymbol{F}^{T} \boldsymbol{P}\right)-\boldsymbol{F}^{T} \operatorname{Div} \boldsymbol{P}-\mathfrak{F} \\
& =-\boldsymbol{F}^{T}[\boldsymbol{f}+\operatorname{Div} \boldsymbol{P}]+\operatorname{Div}\left(\boldsymbol{F}^{T} \boldsymbol{P}\right)-\mathfrak{F} .
\end{aligned}
$$

Moreover, using the Euler-Lagrange equation (67) yields

$$
\operatorname{Grad} W=\operatorname{Div}\left(\boldsymbol{F}^{T} \boldsymbol{P}\right)-\mathfrak{F}
$$

Finally, by virtue of the identity $\operatorname{Grad} W=\operatorname{Div}\left(W \boldsymbol{I}^{T}\right)$, where $\boldsymbol{I}$ is the material identity tensor, (76) becomes

$$
\mathfrak{F}+\operatorname{Div} \mathfrak{E}=\mathbf{0}, \quad \mathfrak{F}_{A}+\mathfrak{E}_{A \mid B}^{B}=0,
$$

where $\mathfrak{E}$ is the Eshelby stress defined as in (49).

Similarly to other field theories, like electromagnetism or general relativity, the tensor $\mathfrak{E}$ defined in (49) plays the role of the ("spatial" part of the) energymomentum tensor of the theory under study. However, we emphasize that, while $\mathfrak{E}$ has been obtained with the aid of a variational argument in the present framework, more general approaches exist, in which $\mathfrak{E}$ is introduced as a primary dynamical quantity [Gurtin 1995]. Equation (77) is called material equilibrium equation or configurational equilibrium equation [Gurtin 1995], by analogy with the equilibrium equation (67) described by the Euler-Lagrange equations.

According to (71), if the body $\mathcal{B}$ is homogeneous, then we have

$$
\mathfrak{F}_{A}(X)=-\left[\frac{\partial \widehat{W}}{\partial X^{A}} \circ(\phi, \boldsymbol{F}, X)\right](X)=0 \quad \text { for all } X \in \mathcal{B},
$$

and (77) implies the vanishing of the divergence of the Eshelby stress. On the contrary, if there is any inhomogeneity in $\mathcal{D}$ (i.e., the derivative $\partial \widehat{W} / \partial X^{A}$ is nonvanishing), this will be captured by the integral of the traction forces $\mathbb{E} N$ of the Eshelby stress over the boundary $\partial \mathcal{D}$.

We now show that (48) yields the weak formulation of the strong form described in (77). This is easy to see by referring to (51), which we obtained from (48) (or (50)) by working in Cartesian coordinates and using Eshelby's displacement $\boldsymbol{U}=-\boldsymbol{U}_{0}$, constant over $\mathcal{D}$. Indeed, by solving the material equilibrium equation (77) for $\mathfrak{F}$, using Cartesian coordinates, integrating over $\mathcal{D}$, applying Gauss's theorem, and contracting both sides with $\boldsymbol{U}_{0}$, we obtain the total configurational force 
on the region $\mathcal{D}$ as the covector $\mathcal{F}$ such that

$$
\mathcal{F} \boldsymbol{U}_{0}=-\left(\int_{\mathcal{D}} \operatorname{Div} \mathfrak{E}\right) \boldsymbol{U}_{0}=-\left(\int_{\partial \mathcal{D}} \mathfrak{E} \boldsymbol{N}\right) \boldsymbol{U}_{0}=\left(\int_{\mathcal{D}} \mathfrak{F}\right) \boldsymbol{U}_{0}
$$

i.e., $\mathcal{F}$ is the integral of the inhomogeneity force density $\mathfrak{F}$, as we see by comparing with (51). Note that, if the body $\mathcal{D}$ is homogeneous, (77) and (78) imply the vanishing of the divergence of the Eshelby stress, and therefore the vanishing of the volume integral and the equivalent surface integral on the right-hand side of (79).

\section{Derivation of the weak form with Noether theorem}

In Noether's theorem, we need to contemporarily transform the domain and perform a variation on the arguments of the Lagrangian. In the jargon of classical field theory, these are called a transformation of the coordinates (material coordinates, in our case) and a variation of the fields, respectively. Together, these give the total variation. We have already shown the transformation of the material coordinates in Section 2.3 and the variation on the fields in Section 4 and we turn now to the total variation. Then, we apply Noether's theorem to directly obtain Eshelby's results. In the application of Noether's theorem, we define the total energy $\mathcal{E}_{\mathcal{D}}$ of a region $\mathcal{D}$ as a functional on the product manifold $\mathcal{C} \times \mathcal{M}$.

5.1. Total variation. In the language of field theory, the total variation is obtained by evaluating the variation of the fields at frozen coordinates given in (57) and (58) at the transformed points $\widetilde{X}=y(X)$, where $y=X+h \boldsymbol{U}: \mathcal{B} \rightarrow \widetilde{\mathcal{B}}$ is the infinitesimal transformation of the coordinates defined in (21), with $U \in T_{X} \mathcal{M}$. In order to avoid confusion, some care must be exercised.

We recall that the manifold $\mathcal{C}$ is the configuration space of the body $\mathcal{B}$, a configuration $\phi$ is an element of $\mathcal{C}$, and a displacement field $\eta$ is a tangent vector of $T_{\phi} \mathcal{C}$. Let us denote by $\tilde{\mathcal{C}}$ the configuration space of the "perturbed" body $\widetilde{\mathcal{B}}=y(\mathcal{B})$, to which the points $\tilde{X}=y(X)$ belong. Consider the intersection $\mathcal{B} \cap \widetilde{\mathcal{B}}$ and the restriction of the configuration $\phi$ and the displacement field $\eta$ defined in a subset $\mathcal{D} \subset \mathcal{B} \cap \widetilde{\mathcal{B}}$ (see Figure 5). In this restriction, it is legitimate to evaluate $\phi$ and $\eta$ at $\widetilde{X}$.

We now define the total variation $\mathcal{C} \rightarrow \tilde{\mathcal{C}}: \phi \mapsto \bar{\phi}$ by evaluating the variations of the fields at frozen coordinates of (57) and (58) at $\widetilde{X} \in \widetilde{\mathcal{B}} \cap \mathcal{B}$, i.e., we define

$$
\begin{aligned}
\bar{\phi}(\tilde{X}) & =\phi(\tilde{X})+h \eta(\tilde{X}), & \bar{\phi}^{a}(\tilde{X}) & =\phi^{a}(\tilde{X})+h \eta^{a}(\tilde{X}), \\
\overline{\boldsymbol{F}}(\tilde{X}) & =\boldsymbol{F}(\tilde{X})+h(\operatorname{Grad} \eta)(\tilde{X}), & \bar{F}_{A}^{a}(\tilde{X}) & =F_{A}^{a}(\tilde{X})+h \eta_{\mid A}^{a}(\tilde{X}),
\end{aligned}
$$

where $h$ is, with no loss of generality, the same smallness parameter as $y=X+h \boldsymbol{U}$. To obtain the final form of the total variation, we substitute the transformation (21) of the coordinates into the variations on the configuration (80) and on the 


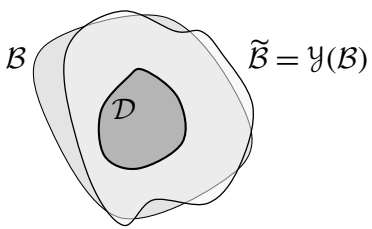

Figure 5. A domain $\mathcal{D}$ (dark gray) in the intersection $\mathcal{B} \cap \widetilde{\mathcal{B}}$ between the body $\mathcal{B}$ (solid gray) and the perturbed body $\widetilde{\mathcal{B}}$ (transparent gray).

tangent (81) of the configuration, respectively, and use Taylor expansion. For the configuration, we have

$$
\begin{aligned}
\bar{\phi}(\tilde{X}) & =\phi(X+h \boldsymbol{U}(X))+h \boldsymbol{\eta}(X+h \boldsymbol{U}(X)) \\
& =\phi(X)+h \boldsymbol{F}(X) \boldsymbol{U}(X)+h \boldsymbol{\eta}(X)+o(h), \\
\bar{\phi}^{a}(\tilde{X}) & =\phi^{a}(X+h \boldsymbol{U}(X))+h \eta^{a}(X+h \boldsymbol{U}(X)) \\
& =\phi^{a}(X)+h F_{B}^{a}(X) U^{B}(X)+h \eta^{a}(X)+o(h),
\end{aligned}
$$

from which, using $\bar{\phi}(\tilde{X})=\bar{\phi}(y(X))=(\bar{\phi} \circ y)(X)$ and omitting the argument $X$, we have

$$
\begin{aligned}
\bar{\phi} \circ y & =\phi+h(\boldsymbol{\eta}+\boldsymbol{F} \boldsymbol{U})+o(h) \quad=\phi+h \boldsymbol{w}+o(h), \\
\bar{\phi}^{a} \circ y & =\phi^{a}+h\left(\eta^{a}+F_{B}^{a} U^{B}\right)+o(h)=\phi^{a}+h w^{a}+o(h),
\end{aligned}
$$

where

$$
\boldsymbol{w}=\boldsymbol{\eta}+\boldsymbol{F} \boldsymbol{U}, \quad w^{a}=\eta^{a}+F_{B}^{a} U^{B} .
$$

For the tangent map, we have

$$
\begin{aligned}
\overline{\boldsymbol{F}}(\tilde{X}) & =\boldsymbol{F}(X+h \boldsymbol{U}(X))+h(\operatorname{Grad} \boldsymbol{\eta})(X+h \boldsymbol{U}(X)) \\
& =\boldsymbol{F}(X)+h(\operatorname{Grad} \boldsymbol{F})(X) \boldsymbol{U}(X)+h(\operatorname{Grad} \boldsymbol{\eta})(X)+o(h), \\
\bar{F}_{A}^{a}(\widetilde{X}) & =F_{A}^{a}(X+h \boldsymbol{U}(X))+h \eta_{\mid A}^{a}(X+h \boldsymbol{U}(X)) \\
& =F_{A}^{a}(X)+h F_{A \mid B}^{a}(X) U^{B}(X)+h \eta_{\mid A}^{a}(X)+o(h),
\end{aligned}
$$

and thus,

$$
\begin{aligned}
\overline{\boldsymbol{F}} \circ y & =\boldsymbol{F}+h(\operatorname{Grad} \boldsymbol{\eta}+(\operatorname{Grad} \boldsymbol{F}) \boldsymbol{U})+o(h)=\boldsymbol{F}+h \boldsymbol{Y}+o(h), \\
\bar{F}_{A}^{a} \circ y & =F_{A}^{a}+h\left(\eta_{\mid A}^{a}+F_{A \mid B}^{a} U^{B}\right)+o(h) \quad=F_{A}^{a}+h Y_{A}^{a}+o(h),
\end{aligned}
$$

where

$$
\boldsymbol{Y}=\operatorname{Grad} \boldsymbol{\eta}+(\operatorname{Grad} \boldsymbol{F}) \boldsymbol{U}, \quad Y_{A}^{a}=\eta_{\mid A}^{a}+F_{A \mid B}^{a} U^{B} .
$$


5.2. Variation of the total energy. Since we are working in the static case, we replace the action functional and the Lagrangian density with the total energy functional $\mathcal{E}$ and the potential energy density $W$. The total energy in a subset $\mathcal{D} \subset \mathcal{B} \cap \widetilde{\mathcal{B}}$ is a functional on the product manifold $\mathcal{C} \times \mathcal{M}$, i.e.,

$$
\mathcal{E}_{\mathcal{D}}: \mathcal{C} \times \mathcal{M} \rightarrow \mathbb{R}:(\phi, y) \mapsto \mathcal{E}_{\mathcal{D}}(\phi, y)=\int_{y_{(\mathcal{D})}} \widehat{W} \circ(\phi, \boldsymbol{F}, X),
$$

where the integration domain $y(\mathcal{D})$ must belong to the intersection $\mathcal{B} \cap \widetilde{\mathcal{B}}$. We now consider the coordinate transformation $y=X+h \boldsymbol{U}$, where $\boldsymbol{U} \in T_{X} \mathcal{M}$ is a tangent vector at the identity $\mathcal{X}$, and the field transformation is $\bar{\phi}=\phi+h \eta$, where $\eta \in T_{\phi} \mathcal{C}$ is a tangent vector at the configuration $\phi$. The variation of the energy is given by the directional derivative

$$
\begin{aligned}
\left(\partial_{(\boldsymbol{\eta}, \boldsymbol{U})} \mathcal{E}_{\mathcal{D}}\right)(\phi, X) & =\lim _{h \rightarrow 0} \frac{\mathcal{E}_{\mathcal{D}}(\bar{\phi}, y)-\mathcal{E}_{\mathcal{D}}(\phi, X)}{h} \\
& =\lim _{h \rightarrow 0} \frac{1}{h}\left[\int_{y(\mathcal{D})} \widehat{W} \circ(\bar{\phi}, \overline{\boldsymbol{F}}, X)-\int_{\mathcal{D}} \widehat{W} \circ(\phi, \boldsymbol{F}, X)\right],
\end{aligned}
$$

evaluated at the conventional configuration $\phi$ and Eshelbian configuration $\mathcal{X}$, with respect to the pair of tangent vectors $(\boldsymbol{\eta}, \boldsymbol{U}) \in T_{(\phi, X)}(\mathcal{C} \times \mathcal{M})$ in the product manifold $\mathcal{C} \times \mathcal{M}$. Note also that, in the second integral, we used $X(\mathcal{D})=\mathcal{D}$.

Application of the theorem of the change of variables on the first integral in (89) yields

$$
\int_{y(\mathcal{D})} \widehat{W} \circ(\bar{\phi}, \overline{\boldsymbol{F}}, X)=\left[\int_{\mathcal{D}}(1+h \operatorname{Div} \boldsymbol{U}) \widehat{W} \circ(\bar{\phi}, \overline{\boldsymbol{F}}, X) \circ y\right]+o(h),
$$

where the determinant $\operatorname{det}(T y)=1+h \operatorname{Div} \boldsymbol{U}+o(h)$ follows from (24). We now notice that

$$
\begin{aligned}
\widehat{W} \circ(\bar{\phi}, \overline{\boldsymbol{F}}, x) \circ y & =\widehat{W} \circ(\bar{\phi} \circ y, \overline{\boldsymbol{F}} \circ y, x \circ y) \\
& =\widehat{W} \circ(\phi+h \boldsymbol{w}+o(h), \boldsymbol{F}+h \boldsymbol{Y}+o(h), x+h \boldsymbol{U}),
\end{aligned}
$$

where we made use of the total variations (83) and (86), as well as of the identity $x \circ y=y=x+h \boldsymbol{U}$. Now, we expand in Taylor series up to the first order, and obtain

$$
\begin{aligned}
\widehat{W} \circ(\bar{\phi}, \overline{\boldsymbol{F}}, X) \circ y=\widehat{W} \circ(\phi, \boldsymbol{F}, X) & +\frac{\partial \widehat{W}}{\partial \phi^{a}} \circ(\phi, \boldsymbol{F}, X) h w^{a} \\
& +\frac{\partial \widehat{W}}{\partial F_{A}^{a}} \circ(\phi, \boldsymbol{F}, X) h Y_{A}^{a} \\
& +\frac{\partial \widehat{W}}{\partial X^{B}} \circ(\phi, \boldsymbol{F}, X) h U^{B}+o(h) .
\end{aligned}
$$


Using (90), (91), and (92) in the variation of the energy (89), we have

$$
\begin{aligned}
\left(\partial_{(\boldsymbol{\eta}, \boldsymbol{U})} \mathcal{E}_{\mathcal{D}}\right)(\phi, X) & =\lim _{h \rightarrow 0} \frac{1}{h}\left[\int _ { \mathcal { D } } h \left(\widehat{W} \circ(\phi, \boldsymbol{F}, X) U_{\mid B}^{B}+\frac{\partial \widehat{W}}{\partial \phi^{a}} \circ(\phi, \boldsymbol{F}, X) w^{a}\right.\right. \\
& \left.\left.+\frac{\partial \widehat{W}}{\partial F_{A}^{a}} \circ(\phi, \boldsymbol{F}, X) Y_{A}^{a}+\frac{\partial \widehat{W}}{\partial X^{B}} \circ(\phi, \boldsymbol{F}, X) U^{B}\right)+o(h)\right] .
\end{aligned}
$$

The smallness parameter cancels out and the term $o(h)$ disappears in the limit $h \rightarrow 0$. Thus, we write

$$
\begin{aligned}
\left(\partial_{(\boldsymbol{\eta}, \boldsymbol{U})} \mathcal{E}_{\mathcal{D}}\right)(\phi, X)=\int_{\mathcal{D}}\left(\widehat{W} \circ(\phi, \boldsymbol{F}, X) U_{\mid B}^{B}+\frac{\partial \widehat{W}}{\partial \phi^{a}} \circ(\phi, \boldsymbol{F}, X) w^{a}\right. \\
\left.+\frac{\partial \widehat{W}}{\partial F_{A}^{a}} \circ(\phi, \boldsymbol{F}, X) Y_{A}^{a}+\frac{\partial \widehat{W}}{\partial X^{B}} \circ(\phi, \boldsymbol{F}, X) U^{B}\right),
\end{aligned}
$$

and we use the explicit expressions (84) and (87) of the total variations $\boldsymbol{w}$ and $\boldsymbol{Y}$ :

$$
\begin{array}{r}
\left(\partial_{(\boldsymbol{\eta}, \boldsymbol{U})} \mathcal{E}_{\mathcal{D}}\right)(\phi, X)=\int_{\mathcal{D}}\left(\widehat{W} \circ(\phi, \boldsymbol{F}, \mathcal{X}) U_{\mid B}^{B}+\frac{\partial \widehat{W}}{\partial \phi^{a}} \circ(\phi, \boldsymbol{F}, \mathcal{X})\left(\eta^{a}+F_{B}^{a} U^{B}\right)\right. \\
\left.+\frac{\partial \widehat{W}}{\partial F_{A}^{a}} \circ(\phi, \boldsymbol{F}, X)\left(\eta_{\mid A}^{a}+F_{A \mid B}^{a} U^{B}\right)+\frac{\partial \widehat{W}}{\partial X^{B}} \circ(\phi, \boldsymbol{F}, X) U^{B}\right) .
\end{array}
$$

Since

$$
\begin{aligned}
& (\widehat{W} \circ(\phi, \boldsymbol{F}, X))_{, B} \\
& \quad=\frac{\partial \widehat{W}}{\partial \phi^{a}} \circ(\phi, \boldsymbol{F}, X) F_{B}^{a}+\frac{\partial \widehat{W}}{\partial F_{A}^{a}} \circ(\phi, \boldsymbol{F}, X) F_{A \mid B}^{a}+\frac{\partial \widehat{W}}{\partial X^{A}} \circ(\phi, \boldsymbol{F}, X) \delta_{B}^{A},
\end{aligned}
$$

we have

$$
\begin{aligned}
\left(\partial_{(\eta, U)} \mathcal{E}_{\mathcal{D}}\right)(\phi, X)=\int_{\mathcal{D}}(\widehat{W} \circ & (\phi, \boldsymbol{F}, X) U_{\mid B}^{B}+(\widehat{W} \circ(\phi, \boldsymbol{F}, X))_{, B} U^{B} \\
& \left.+\frac{\partial \widehat{W}}{\partial \phi^{a}} \circ(\phi, \boldsymbol{F}, X) \eta^{a}+\frac{\partial \widehat{W}}{\partial F_{A}^{a}} \circ(\phi, \boldsymbol{F}, X) \eta_{\mid A}^{a}\right) .
\end{aligned}
$$

Using Leibniz's rule in the first two terms and in the last two terms and separating the integrals, we have

$$
\begin{aligned}
\left(\partial_{(\boldsymbol{\eta}, \boldsymbol{U})} \mathcal{E}_{\mathcal{D}}\right)(\phi, X)=\int_{\mathcal{D}} & {\left[\left(\widehat{W} \circ(\phi, \boldsymbol{F}, X) U^{B}\right)_{\mid B}+\left(\frac{\partial \widehat{W}}{\partial F_{A}^{a}} \circ(\phi, \boldsymbol{F}, X) \eta^{a}\right)_{\mid A}\right] } \\
+ & \int_{\mathcal{D}}\left[\frac{\partial \widehat{W}}{\partial \phi^{a}} \circ(\phi, \boldsymbol{F}, X)-\left(\frac{\partial \widehat{W}^{a}}{\partial F_{A}^{a}} \circ(\phi, \boldsymbol{F}, X)\right)_{\mid A}\right] \eta^{a} .
\end{aligned}
$$

Now we use the definitions (61), which, in the context of continuum mechanics, give the body force $\boldsymbol{f}$ and the first Piola-Kirchhoff stress $\boldsymbol{P}$, use $W=\widehat{W} \circ(\phi, \boldsymbol{F}, X)$, 
and change index $A$ into $B$ in the first integral. So, we have

$$
\left(\partial_{(\eta, U)} \mathcal{E}_{\mathcal{D}}\right)(\phi, X)=\int_{\mathcal{D}}\left[\left(W U^{B}\right)_{\mid B}+\left(\eta^{a} P_{a}{ }^{B}\right)_{\mid B}\right]-\int_{\mathcal{D}}\left(f_{a}+P_{a \mid A}{ }^{A}\right) \eta^{a},
$$

which corresponds to equation (17) in the paper by Hill [1951]. In the first integral, we use $U^{B}=U^{A} \delta_{A}{ }^{B}$ in the first term and the definition (83) of the total variation $\boldsymbol{w}$ to eliminate $\eta^{a}=w^{a}-F_{A}^{a} U^{A}$ in the second term, and then we split the first integral into two, to obtain

$$
\begin{aligned}
\left(\partial_{(\boldsymbol{\eta}, \boldsymbol{U})} \mathcal{E}_{\mathcal{D}}\right)(\phi, X)=\int_{\mathcal{D}}\left[U ^ { A } \left(W \delta_{A}{ }^{B}\right.\right. & \left.\left.-F_{A}^{a} P_{a}{ }^{B}\right)\right]_{\mid B} \\
& +\int_{\mathcal{D}}\left(w^{a} P_{a}{ }^{B}\right)_{\mid B}-\int_{\mathcal{D}}\left(f_{a}+P_{a}{ }^{A} \mid A\right) \eta^{a},
\end{aligned}
$$

where we recognize the Eshelby stress $\mathfrak{E}_{A}{ }^{B}=W \delta_{A}{ }^{B}-F_{A}^{a} P_{a}{ }^{B}$ defined in (49). Finally, we obtain

$$
\left(\partial_{(\boldsymbol{\eta}, \boldsymbol{U})} \mathcal{E}_{\mathcal{D}}\right)(\phi, X)=\int_{\mathcal{D}}\left(U^{A} \mathfrak{E}_{A}{ }^{B}\right)_{\mid B}+\int_{\mathcal{D}}\left(w^{a} P_{a}{ }^{B}\right)_{\mid B}-\int_{\mathcal{D}}\left(f_{a}+P_{a \mid A}{ }^{A}\right) \eta^{a},
$$

which, in component-free formalism, reads

$$
\left(\partial_{(\boldsymbol{\eta}, \boldsymbol{U})} \mathcal{E}_{\mathcal{D}}\right)(\phi, X)=\int_{\mathcal{D}} \operatorname{Div}\left(\mathfrak{E}^{T} \boldsymbol{U}\right)+\int_{\mathcal{D}} \operatorname{Div}\left(\boldsymbol{P}^{T} \boldsymbol{w}\right)-\int_{\mathcal{D}}(\boldsymbol{f}+\operatorname{Div} \boldsymbol{P}) \boldsymbol{\eta} .
$$

If the variation (102) is evaluated for a configuration $\phi$ solving the Euler-Lagrange equations (67), we obtain

$$
\left(\partial_{(\eta, U)} \mathcal{E}_{\mathcal{D}}\right)(\phi, X)=\int_{\mathcal{D}} \operatorname{Div}\left(\mathfrak{E}^{T} \boldsymbol{U}\right)+\int_{\mathcal{D}} \operatorname{Div}\left(\boldsymbol{P}^{T} \boldsymbol{w}\right),
$$

where the first two integrals contain the contributions to the Noether current density $\mathfrak{E}^{T} \boldsymbol{U}+\boldsymbol{P}^{T} \boldsymbol{w}$. The extension of the result (103) to the case of the presence of nonintegrable body forces $\boldsymbol{f}$ is treated in Appendix A.

5.3. Eshelby's results and conservation of Noether's current. The variational procedure followed in Section 5.2 was conducted by introducing the one-parameter families of transformations $y(X)=X+h \boldsymbol{U}=\tilde{X}$ and $\bar{\phi}(\widetilde{X})=\phi(\widetilde{X})+h \eta(\widetilde{X})$, which allowed us to compute the Gâteaux derivative of total energy $\mathcal{E}_{\mathcal{D}}$ along the pair of directions $(\boldsymbol{\eta}, \boldsymbol{U})$. Transformations of this kind are said to be symmetries if they do not alter the numerical value of $\varepsilon_{\mathcal{D}}$, i.e., if it holds true that $\mathcal{E}_{\mathcal{D}}(\bar{\phi}, y)=\mathcal{E}_{\mathcal{D}}(\phi, X)$ for sufficiently small values of $h$. Following an argument reported by Hill [1951], a condition ensuring the compliance with this equality and the form-invariance of the Euler-Lagrange equations is obtained by means of what in field theory is called a divergence transformation [Hill 1951; Maugin 1993]. For the case of an 
infinitesimal symmetry transformation, the divergence transformation reads

$$
\int_{\mathcal{D}}(1+h \operatorname{Div} \boldsymbol{U}) \widehat{W} \circ(\bar{\phi}, \overline{\boldsymbol{F}}, X) \circ y=\int_{\mathcal{D}}[\widehat{W} \circ(\phi, \boldsymbol{F}, X)+h \operatorname{Div} \boldsymbol{\Omega}],
$$

where $\boldsymbol{\Omega}=\widehat{\boldsymbol{\Omega}} \circ X$ is a vector field to be determined. Note that, in order to leave the Euler-Lagrange equations (67) invariant, $\widehat{\boldsymbol{\Omega}}$ must not depend on $\boldsymbol{F}$ [Hill 1951]. By dividing (104) by $h$ and taking the limit for $h \rightarrow 0$, we obtain

$$
\left(\partial_{(\boldsymbol{\eta}, \boldsymbol{U})} \mathcal{E}_{\mathcal{D}}\right)(\phi, X)-\int_{\mathcal{D}} \operatorname{Div} \boldsymbol{\Omega}=\int_{\mathcal{D}}\left[\operatorname{Div}\left(\mathfrak{E}^{T} \boldsymbol{U}\right)+\operatorname{Div}\left(\boldsymbol{P}^{T} \boldsymbol{w}\right)-\operatorname{Div} \boldsymbol{\Omega}\right]=0 .
$$

According to this result, to a given pair $\boldsymbol{U}$ and $\boldsymbol{w}$ there corresponds the conservation law

$$
\operatorname{Div}\left(\mathfrak{E}^{T} \boldsymbol{U}\right)+\operatorname{Div}\left(\boldsymbol{P}^{T} \boldsymbol{w}\right)-\operatorname{Div} \boldsymbol{\Omega}=0,
$$

which allows us to determine $\boldsymbol{\Omega}$. In several circumstances of interest, such as the one related to the conservation of momentum or angular momentum, one can take $\boldsymbol{\Omega}$ to be zero from the outset and look for transformations $\boldsymbol{U}$ and $\boldsymbol{w}$ leading to conservation laws of the form

$$
\operatorname{Div}\left(\mathfrak{E}^{T} \boldsymbol{U}\right)+\operatorname{Div}\left(\boldsymbol{P}^{T} \boldsymbol{w}\right)=0 .
$$

In the remainder of our work, we specialize to this case in order to retrieve Eshelby's result in light of Noether's theorem. Some remarks on divergence transformations are reported in Appendix B.

Eshelby [1975] imposed $\boldsymbol{\eta}=-\boldsymbol{F} \boldsymbol{U}$, i.e., that the conventional displacement $\boldsymbol{\eta}$ be equal to the negative of the push-forward of the material displacement $\boldsymbol{U}$, as shown in (41), in order to preserve compatibility. This condition, in turn, imposes the vanishing of the total variation, i.e., $\boldsymbol{w}=\boldsymbol{\eta}+\boldsymbol{F} \boldsymbol{U}=\mathbf{0}$. With this hypothesis, the integral of $\operatorname{Div}\left(\boldsymbol{P}^{T} \boldsymbol{w}\right)$ in (103) vanishes identically and the variation reduces to

$$
\left(\partial_{(\boldsymbol{\eta}, \boldsymbol{U})} \mathcal{E}_{\mathcal{D}}\right)(\phi, X)=\int_{\mathcal{D}} \operatorname{Div}\left(\mathfrak{E}^{T} \boldsymbol{U}\right),
$$

which coincides with the result shown in (48).

Now we can exploit Noether's theorem to obtain Eshelby's final result. Noether's theorem states:

For every continuous symmetry under which the integral $\mathcal{E}_{\mathcal{D}}$ is invariant, there is a conserved current density.

In this case, the Noether current density is $\mathfrak{E}^{T} \boldsymbol{U}$. For it to be conserved, the divergence $\operatorname{Div}\left(\mathfrak{E}^{T} \boldsymbol{U}\right)$ has to vanish and, in fact, a direct computation, in which the configurational force balance (77) is used, yields the condition

$$
\operatorname{Div}\left(\mathfrak{E}^{T} \boldsymbol{U}\right)=\mathfrak{E}: \operatorname{Grad} \boldsymbol{U}+(\operatorname{Div} \mathfrak{E}) \boldsymbol{U}=\mathfrak{E}: \operatorname{Grad} \boldsymbol{U}-\mathfrak{F} \boldsymbol{U}=0 .
$$


Equation (109) is known as Noetherian identity [Podio-Guidugli 2001], and places restrictions on the class of transformations $\boldsymbol{U}$ that comply with the requirement $\operatorname{Div}\left(\mathfrak{E}^{T} \boldsymbol{U}\right)=0$, which can thus be said to be symmetry transformations. Indeed, a field $\boldsymbol{U}$ is a symmetry transformation (i.e., it leaves $\mathcal{E}_{\mathcal{D}}$ invariant) if, and only if, it satisfies (109) (for a similar result in a different context, see also [Grillo et al. 2003; 2019]). Looking at (109), we notice that, when the inhomogeneity force, $\mathfrak{F}$, vanishes identically, i.e., when the body is materially homogeneous and, thus, the energy density $\widehat{W}$ does not depend on the material points, the Noetherian identity reduces to

$$
\operatorname{Div}\left(\mathfrak{E}^{T} \boldsymbol{U}\right)=\mathfrak{E}: \operatorname{Grad} \boldsymbol{U}=0 .
$$

This result implies that any arbitrary uniform displacement field $\boldsymbol{U}$, for which $\operatorname{Grad} \boldsymbol{U}=\mathbf{0}$, annihilates the divergence of the Noether current density and is, thus, a symmetry transformation. A body endowed with this property is said to enjoy the symmetry of material homogeneity. We notice, however, that, when $\mathfrak{F}$ is not null, $\boldsymbol{U}$ may no longer be uniform. This means that $\mathfrak{F}$ breaks the symmetry of material homogeneity and a new class of transformations $\boldsymbol{U}$ has to be determined.

We also note that, under the hypothesis of homogeneous material, (108) implies the vanishing of the divergence of $\mathfrak{E}^{T} \boldsymbol{U}$, and not of $\mathfrak{E}$. In order to obtain the vanishing of the divergence of the Eshelby stress $\mathfrak{E}$, we implement the last of Eshelby's hypotheses, namely the fact that the material displacement $\boldsymbol{U}$ is uniform on $\mathcal{D}$ and given by $\boldsymbol{U}(X)=-\boldsymbol{U}_{0}$, for every $X \in \mathcal{D}$. This implies that in the integral of $\operatorname{Div}\left(\mathfrak{E}^{T} \boldsymbol{U}\right)$ in (111), the displacement $\boldsymbol{U}=-\boldsymbol{U}_{0}$ can be brought out of the divergence, i.e.,

$$
\left(\partial_{\left(\boldsymbol{\eta},-\boldsymbol{U}_{0}\right)} \mathcal{E}_{\mathcal{D}}\right)(\phi, X)=-\int_{\mathcal{D}}(\operatorname{Div} \mathfrak{E}) \boldsymbol{U}_{0}
$$

which coincides with (50) obtained using Eshelby's original procedure. Now, the vanishing of the variation due to the homogeneity of the material implies the vanishing of Div $\mathfrak{E}$, as in the strong form (77) considered with condition (78).

\section{Summary}

In this work we systematically reviewed the two procedures proposed by Eshelby to study the effect of inhomogeneity in an elastic body, in the differential geometric picture of continuum mechanics. The first procedure [Eshelby 1951] involves the classical cutting-replacing-welding operations and is mathematically represented by defining the energy as a functional on the manifold $\mathcal{M}$ of the Eshelbian configurations $y$ (which transform the domain $\mathcal{D}$ containing the inclusion/defect), and performing a variation on the coordinates, i.e., a variational derivative made with respect to a material displacement field $\boldsymbol{U}$, seen as a variation of the identity Eshelbian configuration $X$. The second procedure [Eshelby 1975] follows Hamilton's 
principle of stationary action. Accordingly, the energy is defined as a functional on the manifold $\mathcal{C}$ of the conventional configurations $\phi$, and a variation is performed on the fields, i.e., a variational derivative is calculated with respect to a spatial displacement, seen as a variation of the configuration map $\phi$.

The natural manner to unify the two procedures is the use of Noether's theorem, in which a variation on both fields and coordinates (total variation) is used. Indeed, to obtain this result, we defined the energy as a functional on the product manifold $\mathcal{C} \times \mathcal{M}$ of the conventional configurations $\phi$ and the Eshelbian configurations $y$, and performed a variational derivative with respect to the pair $(\boldsymbol{\eta}, \boldsymbol{U})$, which is a variation with respect to the pair $(\phi, X)$. While certainly no additional proof was needed to demonstrate the beauty and generality of Noether's theorem, we find that it is insightful to look at Eshelby's theory of defects from the point of view of Noether's conservation laws.

\section{Appendix A: Monogenic and polygenic forces}

The variational setting adopted in our work serves as a basis for the employment of Noether's theorem (see Section 5), which, for first-order theories, is generally enunciated for a Lagrangian density function depending on "fields and gradients of the fields". Hence, the expression of the energy density used so far, i.e., $W=\widehat{W} \circ(\phi, \boldsymbol{F}, \mathcal{X})$, is meant to replicate, up to the sign, the standard functional dependence of a generic Lagrangian density function, for which Noether's theorem is formulated. In principle, however, neither the introduction of the Eshelby stress tensor nor that of the configurational force density require any variational framework. Indeed, as clearly shown by Gurtin [1995], the existence of these quantities stands on its own, and it necessitates neither the hypothesis of hyperelastic material nor the assumption of body forces descending from a generalized potential density. The Eshelby stress tensor, for instance, is defined also for a generic Cauchy elastic material (for a definition of Cauchy elastic materials, see, e.g., [Ogden 1984]), for which the first Piola-Kirchhoff stress tensor, $\boldsymbol{P}$, cannot be determined by differentiating the body's free energy density with respect to its deformation gradient tensor. In this respect, we recall Gurtin's words [1995]: “ $M y$ derivation of Eshelby's relation is accomplished without recourse to constitutive equations or to a variational principle". Yet, what is referred to as "Eshelby stress tensor" and "configurational force density" within a given theory may well depend on whether or not the body is hyperelastic and the body forces admit a potential.

To focus on the consequences of the existence of such a potential, we consider first a hyperelastic and inhomogeneous material with energy density $W^{\text {el }}:=$ $\breve{W}^{\mathrm{el}} \circ(\boldsymbol{F}, \mathcal{X})$, and subjected to body forces for which no integrability hypothesis is made. Then, following Gurtin's approach [1995], the following configurational 
force balance applies:

$$
\operatorname{Div} \mathfrak{E}^{\mathrm{el}}+\mathfrak{F}^{\mathrm{el}}=\mathbf{0},
$$

where $\mathfrak{E}^{\mathrm{el}}:=W^{\mathrm{el}} \boldsymbol{I}^{T}-\boldsymbol{F}^{T} \boldsymbol{P}$ is the Eshelby stress tensor obtained by using $W^{\mathrm{el}}$ as free energy density, and $\mathfrak{F}^{\mathrm{el}}$ is the configurational force density satisfying (112). Note that, for the sake of a lighter notation, we write $\boldsymbol{F}^{T}$ in lieu of $\boldsymbol{F}^{T} \circ \phi$ throughout this section.

To identify $\mathfrak{F}^{\mathrm{el}}$ from (112), we compute explicitly the divergence of $\mathfrak{E}^{\mathrm{el}}$, while recalling the equilibrium equation $\operatorname{Div} \boldsymbol{P}+\boldsymbol{f}=\mathbf{0}$. Thus, we find

$$
\mathfrak{F}^{\mathrm{el}}=-\operatorname{Div} \mathfrak{E}^{\mathrm{el}}=-\frac{\partial \breve{W}^{\mathrm{el}}}{\partial X} \circ(\boldsymbol{F}, X)-\boldsymbol{F}^{T} \boldsymbol{f},
$$

thereby reaching the conclusion that $\mathfrak{F}^{\text {el }}$ consists of the sum of two contributions, denoted by

$$
\begin{aligned}
\mathfrak{F}^{\mathrm{el}, \mathrm{inh}} & :=-\frac{\partial \breve{W}^{\mathrm{el}}}{\partial \mathcal{X}} \circ(\boldsymbol{F}, \mathcal{X}), \\
\mathfrak{F}^{\mathrm{el}, \mathrm{b}} & :=-\boldsymbol{F}^{T} \boldsymbol{f}
\end{aligned}
$$

and ascribable to the inhomogeneity of the material and to the presence of the body force $\boldsymbol{f}$, respectively. We emphasize that (113), (114a), and (114b) are true regardless of any prescription on the integrability of $f$. Still, without loss of generality, we may assume the splitting $f=f^{\mathrm{p}}+\boldsymbol{f}^{\mathrm{m}}$, where $\boldsymbol{f}^{\mathrm{m}}$ is assumed to admit the generalized energy potential density $W^{\mathrm{m}}=\breve{W}^{\mathrm{m}} \circ(\phi, X)$, such that

$$
\boldsymbol{f}^{\mathrm{m}}=-\frac{\partial \breve{W}^{\mathrm{m}}}{\partial \phi} \circ(\phi, X) .
$$

In the terminology of Lánczos [1970, p. 30], $f^{\mathrm{p}}$ is said to be "polygenic", whereas $f^{\mathrm{m}}$ is referred to as a "monogenic" force density, because it is "generated by a single scalar function", i.e., $\breve{W}^{\mathrm{m}}$.

The splitting $f=f^{\mathrm{p}}+\boldsymbol{f}^{\mathrm{m}}$ and (115) permit us to rewrite $\mathfrak{F}^{\mathrm{el}}$ as

$$
\begin{aligned}
\mathfrak{F}^{\mathrm{el}} & =-\frac{\partial \breve{W}^{\mathrm{el}}}{\partial \mathcal{X}} \circ(\boldsymbol{F}, X)-\boldsymbol{F}^{T} \boldsymbol{f} \\
& =-\frac{\partial \breve{W}^{\mathrm{el}}}{\partial \mathcal{X}} \circ(\boldsymbol{F}, X)+\boldsymbol{F}^{T}\left[\frac{\partial \breve{W}^{\mathrm{m}}}{\partial \phi} \circ(\phi, X)\right]-\boldsymbol{F}^{T} \boldsymbol{f}^{\mathrm{p}},
\end{aligned}
$$

and, since it holds true that

$$
\operatorname{Grad} W^{\mathrm{m}}=\boldsymbol{F}^{T}\left[\frac{\partial \breve{W}^{\mathrm{m}}}{\partial \phi} \circ(\phi, X)\right]+\frac{\partial \breve{W}^{\mathrm{m}}}{\partial \mathcal{X}} \circ(\phi, X),
$$


the force density $\mathfrak{F}^{\text {el }}$ takes on the expression

$$
\mathfrak{F}^{\mathrm{el}}=-\frac{\partial \breve{W}^{\mathrm{el}}}{\partial X} \circ(\boldsymbol{F}, X)-\frac{\partial \breve{W}^{\mathrm{m}}}{\partial X} \circ(\phi, X)+\operatorname{Grad} W^{\mathrm{m}}-\boldsymbol{F}^{T} \boldsymbol{f}^{\mathrm{p}} .
$$

Moreover, by exploiting the identity $\operatorname{Grad} W^{\mathrm{m}}=\operatorname{Div}\left(W^{\mathrm{m}} \boldsymbol{I}^{T}\right)$, setting

$$
\begin{gathered}
W^{\mathrm{el}}=\breve{W}^{\mathrm{el}} \circ(\boldsymbol{F}, X)=\widehat{W}^{\mathrm{el}} \circ(\phi, \boldsymbol{F}, X), \quad \text { with } \frac{\partial \widehat{W}^{\mathrm{el}}}{\partial \phi} \circ(\phi, \boldsymbol{F}, X)=\mathbf{0}, \\
W^{\mathrm{m}}=\breve{W}^{\mathrm{m}} \circ(\phi, X)=\widehat{W}^{\mathrm{m}} \circ(\phi, \boldsymbol{F}, X), \quad \text { with } \frac{\partial \widehat{W}^{\mathrm{m}}}{\partial \boldsymbol{F}} \circ(\phi, \boldsymbol{F}, X)=\mathbf{0},
\end{gathered}
$$

and defining the overall energy density, $\widehat{W}:=\widehat{W}^{\mathrm{el}}+\widehat{W}^{\mathrm{m}}$, we obtain

$$
\mathfrak{F}^{\mathrm{el}}=-\frac{\partial \widehat{W}}{\partial X} \circ(\phi, \boldsymbol{F}, X)+\operatorname{Div}\left(W^{\mathrm{m}} \boldsymbol{I}^{T}\right)-\boldsymbol{F}^{T} \boldsymbol{f}^{\mathrm{p}} .
$$

Finally, substituting this result into (112) yields

$$
\operatorname{Div}\left(W^{\mathrm{el}} \boldsymbol{I}^{T}-\boldsymbol{F}^{T} \boldsymbol{P}\right)-\frac{\partial \widehat{W}}{\partial \mathcal{X}} \circ(\phi, \boldsymbol{F}, \mathcal{X})+\operatorname{Div}\left(W^{\mathrm{m}} \boldsymbol{I}^{T}\right)-\boldsymbol{F}^{T} \boldsymbol{f}^{\mathrm{p}}=\mathbf{0},
$$

which can be recast in the form

$$
\operatorname{Div}\left(W \boldsymbol{I}^{T}-\boldsymbol{F}^{T} \boldsymbol{P}\right)-\frac{\partial \widehat{W}}{\partial X} \circ(\phi, \boldsymbol{F}, X)-\boldsymbol{F}^{T} \boldsymbol{f}^{\mathrm{p}}=\mathbf{0} .
$$

We recognize that the term under divergence in (122) is the Eshelby stress tensor used in our work, i.e., $\mathfrak{E}=W \boldsymbol{I}^{T}-\boldsymbol{F}^{T} \boldsymbol{P}$, which is constructed with the energy density $W$. Accordingly, the corresponding configurational force is given by

$$
\mathfrak{F}:=-\frac{\partial \widehat{W}}{\partial X} \circ(\phi, \boldsymbol{F}, X)-\boldsymbol{F}^{T} \boldsymbol{f}^{\mathrm{p}}=\mathfrak{F}^{\mathrm{el}}-\operatorname{Grad} W^{\mathrm{m}},
$$

so that (122) returns the configurational force balance Div $\mathfrak{E}+\mathfrak{F}=\mathbf{0}$. In the absence of polygenic forces, i.e., for $\boldsymbol{f}^{\mathrm{p}}=\mathbf{0}$, the form of the configurational force balance is maintained up to the redefinition of $\mathfrak{F}$, which reduces to

$$
\mathfrak{F}:=-\frac{\partial \widehat{W}}{\partial X} \circ(\phi, F, X),
$$

a result stating that the inhomogeneity force $\mathfrak{F}$ acquires the meaning of an effective force accounting for two contributions: the inhomogeneities of the material featuring in the body's hyperelastic behavior and, thus, represented by $W^{\mathrm{el}}$, and the inhomogeneities of the energy density $W^{\mathrm{m}}$, which describes the interaction of the body with its surrounding world (e.g., via the mass density). 


\section{Appendix B: Divergence transformation}

Let us consider a field theoretical framework and analyze a static problem, described by the Lagrangian density function $\mathcal{L}=\hat{\mathcal{L}} \circ(\varphi, \operatorname{Grad} \varphi, X)$, in which $\varphi$ is a scalar field (the generalization to the situation in which $\varphi$ is a collection of $N$ scalar fields is straightforward). We emphasize that $\varphi$ is not the deformation here, but only a generic scalar field, as it could be the case for temperature or for the scalar potential in electromagnetism. Consequently, the evaluation $\varphi(X)$, with $X \in \mathcal{B}$, only represents the value taken by $\varphi$ at $X$, i.e., it is not the embedding of the material point $X$ into the three-dimensional Euclidean space. Within this setting, the quantity Grad $\varphi$ need not be the "material gradient" of $\varphi$. Still, we maintain the notation introduced so far in our work in order not to generate confusion.

After renaming $\hat{\mathcal{L}} \equiv \hat{\mathcal{L}}_{\text {old }}$, we express the divergence transformation as [Hill 1951]

$$
\hat{\mathcal{L}}_{\text {new }} \circ(\varphi, \operatorname{Grad} \varphi, X)=\hat{\mathcal{L}}_{\text {old }} \circ(\varphi, \operatorname{Grad} \varphi, X)+\operatorname{Div} \boldsymbol{\Omega},
$$

where $\boldsymbol{\Omega}=\widehat{\boldsymbol{\Omega}} \circ(\varphi, X)$ is an arbitrary vector field. Moreover, we notice that the vector-valued function $\widehat{\boldsymbol{\Omega}}$ has to be independent of $\operatorname{Grad} \varphi$.

A first direct consequence of (125) is that the overall Lagrangian ${ }^{2}$ associated with the body transforms from

$$
L_{\mathcal{B}}^{\text {old }}(\varphi)=\int_{\mathcal{B}}\left[\hat{\mathcal{L}}_{\text {old }} \circ(\varphi, \operatorname{Grad} \varphi, X)\right]
$$

into

$$
L_{\mathcal{B}}^{\text {new }}(\varphi)=\int_{\mathcal{B}}\left[\hat{\mathcal{L}}_{\text {new }} \circ(\varphi, \operatorname{Grad} \varphi, X)\right]
$$

where $L_{\mathcal{B}}^{\text {old }}(\varphi)$ and $L_{\mathcal{B}}^{\text {new }}(\varphi)$ differ from each other by the boundary term $\int_{\partial \mathcal{B}} \boldsymbol{\Omega} \boldsymbol{N}$, i.e.,

$$
L_{\mathcal{B}}^{\text {new }}(\varphi)=L_{\mathcal{B}}^{\text {old }}(\varphi)+\int_{\partial \mathcal{B}}[\widehat{\boldsymbol{\Omega}} \circ(\varphi, X)] \boldsymbol{N}
$$

Since the variational procedure yielding the stationarity conditions for $L_{\mathcal{B}}^{\text {old }}(\varphi)$ and $L_{\mathcal{B}}^{\text {new }}(\varphi)$ requires the fields $\varphi$ and $\bar{\varphi}=\varphi+h \eta$ to coincide with each other on $\partial \mathcal{B}$ (indeed, $\eta$ is chosen such that it vanishes on $\partial \mathcal{B}$ ), a field $\varphi$ for which $L_{\mathcal{B}}^{\text {old }}(\varphi)$ is stationary makes $L_{\mathcal{B}}^{\text {new }}(\varphi)$ stationary too. Moreover, such a field has to satisfy the

\footnotetext{
${ }^{2}$ In a more general - yet conceptually equivalent - framework, we should speak of action functional, rather than "overall Lagrangian", with the former being defined as the time integral of the latter over a given (bounded) time interval. However, since all the quantities introduced in the present work are independent of time because of the hypothesis of static problem, the action and the "overall Lagrangian" are defined up to a multiplicative constant representing the width of the given time interval. For this reason, the formulation used in our work is totally equivalent to the general one.
} 
same set of Euler-Lagrange equations. Indeed, upon recalling the expression of the covariant divergence of $\boldsymbol{\Omega}$, i.e.,

$$
\begin{aligned}
\operatorname{Div} \boldsymbol{\Omega} & =\Omega^{A}{ }_{, A}+\Gamma_{B A}^{A} \Omega^{B} \\
& =\left[\frac{\partial \widehat{\Omega}^{A}}{\partial \varphi} \circ(\varphi, X)\right] \varphi_{, A}+\frac{\partial \widehat{\Omega}^{A}}{\partial X^{A}} \circ(\varphi, X)+\Gamma_{B A}^{A}\left[\widehat{\Omega}^{B} \circ(\varphi, X)\right],
\end{aligned}
$$

and substituting (129) into (125), we find that another consequence of (125) is given by the identities

$$
\begin{gathered}
\frac{\partial \hat{\mathcal{L}}_{\text {new }}}{\partial \varphi} \circ(\cdots)=\frac{\partial \hat{\mathcal{L}}_{\text {old }}}{\partial \varphi} \circ(\cdots)+\left[\frac{\partial^{2} \widehat{\Omega}^{A}}{\partial \varphi^{2}} \circ(\varphi, X)\right] \varphi, A \\
+\frac{\partial^{2} \widehat{\Omega}^{A}}{\partial X^{A} \partial \varphi} \circ(\varphi, X)+\Gamma_{B A}^{A}\left[\frac{\partial \widehat{\Omega}^{B}}{\partial \varphi} \circ(\varphi, X)\right], \\
\frac{\partial \hat{\mathcal{L}}_{\text {new }}}{\partial \varphi, B} \circ(\cdots)=\frac{\partial \hat{\mathcal{L}}_{\text {old }}}{\partial \varphi, B} \circ(\cdots)+\frac{\partial \widehat{\Omega}^{B}}{\partial \varphi} \circ(\varphi, X), \\
{\left[\frac{\partial \hat{\mathcal{L}}_{\text {new }}}{\partial \varphi_{, B}} \circ(\cdots)\right]_{\mid B}=\left[\frac{\partial \hat{\mathcal{L}}_{\text {old }}}{\partial \varphi, B} \circ(\cdots)\right]_{\mid B}+\left[\frac{\partial^{2} \widehat{\Omega}^{B}}{\partial \varphi^{2}} \circ(\varphi, X)\right] \varphi, B} \\
\quad+\frac{\partial^{2} \widehat{\Omega}^{B}}{\partial \varphi \partial X^{B}} \circ(\varphi, X)+\Gamma_{D B}^{B}\left[\frac{\partial \widehat{\Omega}^{D}}{\partial \varphi} \circ(\varphi, X)\right],
\end{gathered}
$$

which imply the invariance of the Euler-Lagrange equations under the transformation (125), i.e.,

$$
\frac{\partial \hat{\mathcal{L}}_{\text {old }}}{\partial \varphi} \circ(\cdots)-\left(\frac{\partial \hat{\mathcal{L}}_{\text {old }}}{\partial \varphi_{, B}} \circ(\cdots)\right)_{\mid B}=\frac{\partial \hat{\mathcal{L}}_{\text {new }}}{\partial \varphi} \circ(\cdots)-\left(\frac{\partial \hat{\mathcal{L}}_{\text {new }}}{\partial \varphi_{, B}} \circ(\cdots)\right)_{\mid B}=0 .
$$

We emphasize that this result holds true because $\operatorname{Div}[\widehat{\boldsymbol{\Omega}} \circ(\varphi, X)]$ solves identically the Euler-Lagrange equations, i.e.,

$$
\frac{\partial}{\partial \varphi} \operatorname{Div}[\widehat{\boldsymbol{\Omega}} \circ(\varphi, X)]-\operatorname{Div}\left(\frac{\partial}{\partial \operatorname{Grad} \varphi} \operatorname{Div}[\widehat{\boldsymbol{\Omega}} \circ(\varphi, X)]\right)=0 .
$$

If $\varphi$ is a collection of $N$ independent scalar fields, (132) becomes a system of $N$ scalar equations, i.e., in components,

$$
\frac{\partial}{\partial \varphi^{\mu}} \operatorname{Div}[\widehat{\boldsymbol{\Omega}} \circ(\varphi, X)]-\left(\frac{\partial}{\partial \varphi_{, A}^{\mu}} \operatorname{Div}\left[\widehat{\boldsymbol{\Omega}}_{\circ}(\varphi, X)\right]\right)_{\mid A}=0, \quad \mu=1, \ldots, N
$$

However, the quantity

$$
\frac{\partial}{\partial \varphi_{, A}^{\mu}} \operatorname{Div}[\widehat{\boldsymbol{\Omega}} \circ(\varphi, X)], \quad \mu=1, \ldots, N, A=1,2,3,
$$


is not, in general, the component of a tensor field. Indeed, if it were, for example for $N=3$, the covariant divergence constituting the second term on the left-hand side of (133) would require us to differentiate the tensors $\boldsymbol{e}^{\mu} \otimes \boldsymbol{E}_{A}$ of a suitable tensor basis, thereby yielding a term, obtained by differentiating $\boldsymbol{e}^{\mu}$, that does not cancel with the first summand of (133). Hence, (133) would not be satisfied.

The situation just depicted occurs when the "fields" of the triplet $\left(\varphi^{1}, \varphi^{2}, \varphi^{3}\right)$ acquire the meaning of the components of the deformation, an object that has the mathematical meaning of an embedding and, thus, that is not truly identifiable with a collection of genuine scalar fields. Indeed, when $\left(\varphi^{1}, \varphi^{2}, \varphi^{3}\right)$ is replaced by $\left(\phi^{1}, \phi^{2}, \phi^{3}\right)$, the corresponding "gradient" is none other than $\boldsymbol{F}$ and, more importantly, the quantity in (134) becomes (with $a \in\{1,2,3\}$ and $A \in\{1,2,3\}$ )

$$
\frac{\partial}{\partial \phi_{, A}^{a}} \operatorname{Div}\left[\widehat{\boldsymbol{\Omega}}_{\circ}(\phi, X)\right]=\frac{\partial}{\partial F_{A}^{a}} \operatorname{Div}[\widehat{\boldsymbol{\Omega}} \circ(\phi, X)],
$$

which takes on the meaning of a fictitious first Piola-Kirchhoff stress tensor. The consequence of this result is that the covariant divergence of the right-hand side of (135) does not cancel with $\partial \operatorname{Div}[\widehat{\boldsymbol{\Omega}} \circ(\phi, X)] / \partial \phi^{a}$. This leads us to the conclusion, already stated by Maugin [1993, p. 100], that $\widehat{\boldsymbol{\Omega}}$ should depend "at most" on $X$ "and not on the fields".

Since we consider a static problem, for which the body's Lagrangian density function coincides with the negative of its total energy density, following Hill [1951], we introduce the functions $W_{\text {old }}=\widehat{W}_{\text {old }} \circ(\phi, \boldsymbol{F}, X)$ and $W_{\text {new }}=\widehat{W}_{\text {new }} \circ$ $(\phi, \boldsymbol{F}, X)$, and we reformulate the transformation (125) as

$$
-\widehat{W}_{\text {new }} \circ(\phi, \boldsymbol{F}, X)=-\widehat{W}_{\text {old }} \circ(\phi, \boldsymbol{F}, X)+\operatorname{Div} \boldsymbol{\Omega},
$$

with $\boldsymbol{\Omega} \equiv \widehat{\boldsymbol{\Omega}} \circ \mathcal{X}$. For the reasons outlined above, the divergence transformation (136) is such that the overall energies $\mathcal{E}_{\mathcal{D}}^{\text {old }}(\phi)=\int_{\mathcal{D}} \widehat{W}_{\text {old }} \circ(\phi, \boldsymbol{F}, X)$ and $\varepsilon_{\mathcal{D}}^{\text {new }}(\phi)=$ $\int_{\mathcal{D}} \widehat{W}_{\text {new }} \circ(\phi, \boldsymbol{F}, X)$ are stationary for the same deformation $\phi$, which thus satisfies the same Euler-Lagrange equations. Indeed, since $\widehat{\boldsymbol{\Omega}}$ is independent of $\phi$, it holds true that

$$
\begin{gathered}
\frac{\partial \widehat{W}_{\text {new }}}{\partial \phi^{b}} \circ(\cdots)=\frac{\partial \widehat{W}_{\text {old }}}{\partial \phi^{b}} \circ(\cdots) \\
\frac{\partial \widehat{W}_{\text {new }}}{\partial F_{B}^{b}} \circ(\cdots)=\frac{\partial \widehat{W}_{\text {old }}}{\partial F_{B}^{b}} \circ(\cdots) \\
\frac{\partial \widehat{W}_{\text {old }}}{\partial \phi^{b}} \circ(\cdots)-\left(\frac{\partial \widehat{W}_{\text {old }}}{\partial F_{B}^{b}} \circ(\cdots)\right)_{\mid B}=\frac{\partial \widehat{W}_{\text {new }}}{\partial \phi^{b}} \circ(\cdots)-\left(\frac{\partial \widehat{W}_{\text {new }}}{\partial F_{B}^{b}} \circ(\cdots)\right)_{\mid B}=0 .
\end{gathered}
$$

After proving this property, we superimpose the transformations $X \mapsto \widetilde{X}=y(X)=$ $X+h \boldsymbol{U}$ and $\phi(X) \mapsto \bar{\phi}(\widetilde{X})=\phi(\widetilde{X})+h \eta(\widetilde{X})$ to the divergence transformation (136), 
and we require the invariance of the overall energy under the resulting, global transformation [Hill 1951]. This yields the equality

$$
\underbrace{\int_{\mathcal{D}}\left\{\left[\widehat{W}_{\text {new }} \circ(\bar{\phi}, \overline{\boldsymbol{F}}, \mathcal{X})\right] \circ y\right\} \operatorname{det}(T y)}_{\equiv \mathcal{E}_{\mathcal{D}}^{\text {new }}(\bar{\phi}, y)}=\underbrace{\int_{\mathcal{D}} \widehat{W}_{\text {old }} \circ(\phi, \boldsymbol{F}, X)}_{\equiv \varepsilon_{\mathcal{D}}^{\text {old }}(\phi, x)},
$$

where $T y$ is the tangent map of $y$. By applying a "rescaled" divergence transformation to the left-hand side of (138), i.e.,

$$
\widehat{W}_{\text {new }} \circ(\bar{\phi}, \overline{\boldsymbol{F}}, X)=\widehat{W}_{\text {old }} \circ(\bar{\phi}, \overline{\boldsymbol{F}}, X)-\operatorname{Div}(h \boldsymbol{\Omega}),
$$

we obtain

$$
\int_{\mathcal{D}}\left\{\left[\widehat{W}_{\text {old }} \circ(\bar{\phi}, \overline{\boldsymbol{F}}, X)\right] \circ y-\operatorname{Div}(h \boldsymbol{\Omega}) \circ y\right\} \operatorname{det}(T y)=\int_{\mathcal{D}} \widehat{W}_{\text {old }} \circ(\phi, \boldsymbol{F}, X) .
$$

We remark that the smallness parameter $h$, which multiplies $\boldsymbol{\Omega}$ in (139) and (140), has been introduced in order to make the divergence transformation infinitesimal, as is the case for the transformations on the material points and on the deformation.

By rearranging (140), so as to separate the transformations on the material points and on the deformation from the divergence transformation, we find

$$
\int_{\mathcal{D}}\left\{\left[\widehat{W}_{\text {old }} \circ(\bar{\phi}, \overline{\boldsymbol{F}}, X) \circ y\right] \operatorname{det}(T y)-\widehat{W}_{\text {old }} \circ(\phi, \boldsymbol{F}, X)\right\}=\int_{\mathcal{D}}[\operatorname{Div}(h \boldsymbol{\Omega}) \circ y] \operatorname{det}(T y) .
$$

By using the result reported in (103), at the first order in $h,(141)$ becomes

$$
\int_{\mathcal{D}} \operatorname{Div}\left[\mathfrak{E}^{T} \boldsymbol{U}+\boldsymbol{P}^{T} \boldsymbol{w}\right]=\int_{\mathcal{D}} \operatorname{Div} \boldsymbol{\Omega} \Rightarrow \int_{\mathcal{D}} \operatorname{Div}\left[\mathfrak{E}^{T} \boldsymbol{U}+\boldsymbol{P}^{T} \boldsymbol{w}-\boldsymbol{\Omega}\right]=0,
$$

thereby implying that Noether's current density is given by $\mathfrak{J}=\mathfrak{E}^{T} \boldsymbol{U}+\boldsymbol{P}^{T} \boldsymbol{w}-\boldsymbol{\Omega}$ and that, after localization, the conservation laws should be sought for in the form

$$
\operatorname{Div}\left[\mathfrak{E}^{T} \boldsymbol{U}+\boldsymbol{P}^{T} \boldsymbol{w}-\boldsymbol{\Omega}\right]=0 .
$$

The choice of $\boldsymbol{\Omega}$ depends on the type of conservation law and on the associated class of symmetry which one is interested in looking at.

Within the present context, (143) constitutes the most general form of conservation law pertaining to Noether's current. This result, however, can be exploited in much deeper detail: indeed, granted the Euler-Lagrange equations $\boldsymbol{f}+\operatorname{Div} \boldsymbol{P}=\mathbf{0}$, if, for a given choice of the fields $\boldsymbol{U}, \boldsymbol{w}$, and $\boldsymbol{\Omega},(143)$ is satisfied as an identity, then a specific physical quantity is conserved and the fields are said to be symmetries. 
For the problem under investigation, (143) can be recast in the equivalent form [Hill 1951; Grillo et al. 2003; 2019]

$$
\begin{aligned}
\operatorname{Div}\left[\mathfrak{E}^{T} \boldsymbol{U}+\boldsymbol{P}^{T} \boldsymbol{w}-\boldsymbol{\Omega}\right] & =(\operatorname{Div} \mathfrak{E}) \boldsymbol{U}+\mathfrak{E}: \operatorname{Grad} \boldsymbol{U}+(\operatorname{Div} \boldsymbol{P}) \boldsymbol{w}+\boldsymbol{P}: \operatorname{Grad} \boldsymbol{w}-\operatorname{Div} \boldsymbol{\Omega} \\
& =-\mathfrak{F} \boldsymbol{U}+\mathfrak{E}: \operatorname{Grad} \boldsymbol{U}-\boldsymbol{f} \boldsymbol{w}+\boldsymbol{P}: \operatorname{Grad} \boldsymbol{w}-\operatorname{Div} \boldsymbol{\Omega}=0 .
\end{aligned}
$$

If one is interested in looking at the conservation of linear momentum, one sets $\boldsymbol{U}=\mathbf{0}, \boldsymbol{\Omega}=\mathbf{0}$, and $\boldsymbol{w}=\boldsymbol{w}_{0}$, with $\boldsymbol{w}_{0}$ being a uniform displacement field. In this case, (144) is not satisfied. Indeed, it occurs that

$$
\operatorname{Div}\left[\mathfrak{E}^{T} \boldsymbol{U}+\boldsymbol{P}^{T} \boldsymbol{w}-\boldsymbol{\Omega}\right]=\operatorname{Div}\left[\boldsymbol{P}^{T} \boldsymbol{w}_{0}\right]=-\boldsymbol{f} \boldsymbol{w}_{0} \neq 0,
$$

which shows that linear momentum is not conserved because of the body forces $f$.

On the same footing, the presence of the inhomogeneity force, $\mathfrak{F}$, spoils the conservation of the pseudomomentum [Maugin 1993], and this is reflected by the fact that uniform translations of material points, hereafter denoted by $\boldsymbol{U}=\boldsymbol{U}_{0}$, are not symmetry transformations. This is encompassed by (144) by setting $\boldsymbol{w}=\mathbf{0}$ and $\boldsymbol{\Omega}=\mathbf{0}$, thereby obtaining

$$
\operatorname{Div}\left[\mathfrak{E}^{T} \boldsymbol{U}+\boldsymbol{P}^{T} \boldsymbol{w}-\boldsymbol{\Omega}\right]=-\mathfrak{F} \boldsymbol{U}_{0} \neq 0 .
$$

In fact, Hill [1951] presents several examples, from which we largely took inspiration, and, among those, he shows that the only case in which $\boldsymbol{\Omega}$ should be taken different from the null vector is the case in which velocity transformations are applied, a situation referred to as the center-of-mass theorem.

\section{Acknowledgements}

Federico was supported by the Natural Sciences and Engineering Research Council of Canada, through the NSERC Discovery Programme, Project no. RGPIN2015-06027, Alhasadi through the Libyan Ministry of Higher Education and Grillo through the Dipartimento di Eccellenza 2018-2022, Politecnico di Torino (Italy), Project no. E11G18000350001. All authors have contributed equally to this work.

\section{References}

[Bonet and Wood 2008] J. Bonet and R. D. Wood, Nonlinear continuum mechanics for finite element analysis, 2nd ed., Cambridge University, 2008.

[Cermelli et al. 2001] P. Cermelli, E. Fried, and S. Sellers, "Configurational stress, yield and flow in rate-independent plasticity", R. Soc. Lond. Proc. A 457:2010 (2001), 1447-1467.

[dell'Isola and Placidi 2011] F. dell'Isola and L. Placidi, "Variational principles are a powerful tool also for formulating field theories", pp. 1-15 in Variational models and methods in solid and fluid mechanics, edited by F. dell'Isola and S. Gavrilyuk, CISM Courses and Lect. 535, Springer, 2011.

[Edelen 1981] D. G. B. Edelen, "Aspects of variational arguments in the theory of elasticity: fact and folklore", Internat. J. Solids and Structures 17:8 (1981), 729-740. 
[Epstein 2002] M. Epstein, "The Eshelby tensor and the theory of continuous distributions of inhomogeneities", Mech. Res. Comm. 29:6 (2002), 501-506.

[Epstein 2009] M. Epstein, "The split between remodelling and aging", Int. J. Nonlinear Mech. 44:6 (2009), 604-609.

[Epstein 2010] M. Epstein, The geometrical language of continuum mechanics, Cambridge University, 2010.

[Epstein 2015] M. Epstein, "Mathematical characterization and identification of remodeling, growth, aging and morphogenesis", J. Mech. Phys. Solids 84 (2015), 72-84.

[Epstein and Elżanowski 2007] M. Epstein and M. Elżanowski, Material inhomogeneities and their evolution: a geometric approach, Springer, 2007.

[Epstein and Maugin 1990] M. Epstein and G. A. Maugin, "The energy-momentum tensor and material uniformity in finite elasticity", Acta Mech. 83:3-4 (1990), 127-133.

[Epstein and Maugin 2000] M. Epstein and G. A. Maugin, "Thermomechanics of volumetric growth in uniform bodies", Int. J. Plasticity 16:7-8 (2000), 951-978.

[Eshelby 1951] J. D. Eshelby, "The force on an elastic singularity", Philos. Trans. Roy. Soc. London. A 244 (1951), 84-112.

[Eshelby 1975] J. D. Eshelby, "The elastic energy-momentum tensor", J. Elasticity 5:3-4 (1975), 321-335.

[Federico 2012] S. Federico, "Covariant formulation of the tensor algebra of non-linear elasticity", Int. J. Nonlinear Mech. 47:2 (2012), 273-284.

[Federico et al. 2016] S. Federico, A. Grillo, and R. Segev, "Material description of fluxes in terms of differential forms", Contin. Mech. Thermodyn. 28:1-2 (2016), 379-390.

[Fletcher 1976] D. C. Fletcher, "Conservation laws in linear elastodynamics", Arch. Rational Mech. Anal. 60:4 (1976), 329-353.

[Fried and Gurtin 1994] E. Fried and M. E. Gurtin, "Dynamic solid-solid transitions with phase characterized by an order parameter", Phys. D 72:4 (1994), 287-308.

[Fried and Gurtin 2004] E. Fried and M. E. Gurtin, "A unified treatment of evolving interfaces accounting for small deformations and atomic transport with emphasis on grain-boundaries and epitaxy", Adv. Appl. Mech. 40 (2004), 1-177.

[Golebiewska Herrmann 1982] A. Golebiewska Herrmann, "Material momentum tensor and pathindependent integrals of fracture mechanics”, Int. J. Solids Struct. 18:4 (1982), 319-326.

[Grillo et al. 2003] A. Grillo, S. Federico, G. Giaquinta, W. Herzog, and G. La Rosa, "Restoration of the symmetries broken by reversible growth in hyperelastic bodies", Theoret. Appl. Mech. 30:4 (2003), 311-331.

[Grillo et al. 2005] A. Grillo, G. Zingali, S. Federico, W. Herzog, and G. Giaquinta, "The role of material inhomogeneities in biological growth", Theoret. Appl. Mech. 32:1 (2005), 21-38.

[Grillo et al. 2016] A. Grillo, R. Prohl, and G. Wittum, "A poroplastic model of structural reorganisation in porous media of biomechanical interest", Contin. Mech. Thermodyn. 28:1-2 (2016), 579-601.

[Grillo et al. 2017] A. Grillo, R. Prohl, and G. Wittum, "A generalised algorithm for anelastic processes in elastoplasticity and biomechanics", Math. Mech. Solids 22:3 (2017), 502-527.

[Grillo et al. 2019] A. Grillo, S. Di Stefano, and S. Federico, "Growth and remodelling from the perspective of Noether's theorem”, Mech. Res. Comm. 97 (2019), 89-95.

[Gurtin 1986] M. E. Gurtin, "Two-phase deformations of elastic solids", pp. 147-175 in The breadth and depth of continuum mechanics, edited by C. Truesdell and J. Serrin, Springer, 1986. 
[Gurtin 1993] M. E. Gurtin, "The dynamics of solid-solid phase transitions, I: Coherent interfaces", Arch. Rational Mech. Anal. 123:4 (1993), 305-335.

[Gurtin 1995] M. E. Gurtin, "The nature of configurational forces", Arch. Rational Mech. Anal. 131:1 (1995), 67-100.

[Gurtin 2000] M. E. Gurtin, Configurational forces as basic concepts of continuum physics, Applied Mathematical Sciences 137, Springer, 2000.

[Gurtin and Podio-Guidugli 1996] M. E. Gurtin and P. Podio-Guidugli, "On configurational inertial forces at a phase interface", J. Elasticity 44:3 (1996), 255-269.

[Hamedzadeh et al. 2019] A. Hamedzadeh, A. Grillo, M. Epstein, and S. Federico, "Remodelling of biological tissues with fibre recruitment and reorientation in the light of the theory of material uniformity", Mech. Res. Comm. 96 (2019), 56-61.

[Hill 1951] E. L. Hill, "Hamilton's principle and the conservation theorems of mathematical physics", Rev. Modern Physics 23 (1951), 253-260.

[Huang and Batra 1996] Y.-N. Huang and R. C. Batra, "Energy-momentum tensors in nonsimple elastic dielectrics", J. Elasticity 42:3 (1996), 275-281.

[Imatani and Maugin 2002] S. Imatani and G. A. Maugin, "A constitutive model for material growth and its application to three-dimensional finite element analysis", Mech. Res. Comm. 29:6 (2002), 477-483.

[Kienzler and Herrmann 2000] R. Kienzler and G. Herrmann, Mechanics in material space: with applications to defect and fracture mechanics, Springer, 2000.

[Knowles and Sternberg 1971] J. K. Knowles and E. Sternberg, "On a class of conservation laws in linearized and finite elastostatics”, Arch. Rational Mech. Anal. 44 (1971), 187-211.

[Lánczos 1970] C. Lánczos, The variational principles of mechanics, 4th ed., Mathematical Expositions 4, University of Toronto, 1970.

[Marsden and Hughes 1983] J. E. Marsden and T. J. R. Hughes, Mathematical foundations of elasticity, Prentice-Hall, Englewood Cliffs, NJ, 1983.

[Maugin 1993] G. A. Maugin, Material inhomogeneities in elasticity, Applied Mathematics and Mathematical Computation 3, Chapman \& Hall, London, 1993.

[Maugin 2011] G. A. Maugin, Configurational forces: thermomechanics, physics, mathematics, and numerics, CRC, Boca Raton, FL, 2011.

[Maugin and Epstein 1998] G. A. Maugin and M. Epstein, "Geometrical material structure of elastoplasticity", Int. J. Plasticity 14:1-3 (1998), 109-115.

[Maugin and Trimarco 1992] G. A. Maugin and C. Trimarco, "Pseudomomentum and material forces in nonlinear elasticity: variational formulations and application to brittle fracture", Acta Mech. 94:1-2 (1992), 1-28.

[Noether 1971] E. Noether, "Invariant variation problems", Transport Theory Statist. Phys. 1:3 (1971), 186-207.

[Noll 1967] W. Noll, "Materially uniform simple bodies with inhomogeneities", Arch. Rational Mech. Anal. 27 (1967), 1-32.

[Ogden 1984] R. W. Ogden, Nonlinear elastic deformations, Ellis Horwood, New York, 1984.

[Olver 1984a] P. J. Olver, "Conservation laws in elasticity, I: General results", Arch. Rational Mech. Anal. 85:2 (1984), 111-129.

[Olver 1984b] P. J. Olver, "Conservation laws in elasticity, II: Linear homogeneous isotropic elastostatics”, Arch. Rational Mech. Anal. 85:2 (1984), 131-160. 
[Podio-Guidugli 2001] P. Podio-Guidugli, "Configurational balances via variational arguments", Interfaces Free Bound. 3:2 (2001), 223-232.

[Segev 2013] R. Segev, "Notes on metric independent analysis of classical fields", Math. Methods Appl. Sci. 36:5 (2013), 497-566.

[Truesdell and Noll 1965] C. Truesdell and W. Noll, The non-linear field theories of mechanics, edited by S. Flügge, Handbuch der Physik III-3, Springer, 1965.

[Verron et al. 2009] E. Verron, M. Aït-Bachir, and P. Castaing, "Some new properties of the Eshelby stress tensor", pp. 27-35 in IUTAM Symposium on Progress in the Theory and Numerics of Configurational Mechanics, edited by P. Steinmann, IUTAM Bookser. 17, Springer, 2009.

[Weng and Wong 2009] G. J. Weng and D. T. Wong, "Thermodynamic driving force in ferroelectric crystals with a rank-2 laminated domain pattern, and a study of enhanced electrostriction", J. Mech. Phys. Solids 57:3 (2009), 571-597.

Received 6 Feb 2019. Revised 15 Jun 2019. Accepted 17 Aug 2019.

SALVATORE FEDERICO: salvatore.federico@ucalgary.ca

Department of Mechanical and Manufacturing Engineering, University of Calgary, Calgary, $A B$ Canada

MAWAFAG F. AlHASAdI: mfalhasa@ucalgary.ca

Department of Mechanical and Manufacturing Engineering, University of Calgary, Calgary, $A B$ Canada

ALFIO GRILlO: alfio.grillo@polito.it

Deptartment of Mathematical Sciences "G.L. Lagrange”, Politecnico di Torino,

Dipartimento di Eccellenza, Torino, Italy 



\section{Guidelines for Authors}

Authors may submit manuscripts in PDF format on-line at the submission page.

Originality. Submission of a manuscript acknowledges that the manuscript is original and and is not, in whole or in part, published or under consideration for publication elsewhere. It is understood also that the manuscript will not be submitted elsewhere while under consideration for publication in this journal.

Language. Articles in MEMOCS are usually in English, but articles written in other languages are welcome.

Required items. A brief abstract of about 150 words or less must be included. It should be selfcontained and not make any reference to the bibliography. If the article is not in English, two versions of the abstract must be included, one in the language of the article and one in English. Also required are keywords and a Mathematics Subject Classification or a Physics and Astronomy Classification Scheme code for the article, and, for each author, postal address, affiliation (if appropriate), and email address if available. A home-page URL is optional.

Format. Authors are encouraged to use $\mathrm{IAT}_{\mathrm{E} X}$ and the standard amsart class, but submissions in other varieties of $\mathrm{T}_{\mathrm{E}} \mathrm{X}$, and exceptionally in other formats, are acceptable. Initial uploads should normally be in PDF format; after the refereeing process we will ask you to submit all source material.

References. Bibliographical references should be complete, including article titles and page ranges. All references in the bibliography should be cited in the text. The use of $\mathrm{BIBT}_{\mathrm{E}} \mathrm{X}$ is preferred but not required. Tags will be converted to the house format, however, for submission you may use the format of your choice. Links will be provided to all literature with known web locations and authors are encouraged to provide their own links in addition to those supplied in the editorial process.

Figures. Figures must be of publication quality. After acceptance, you will need to submit the original source files in vector graphics format for all diagrams in your manuscript: vector EPS or vector PDF files are the most useful.

Most drawing and graphing packages - Mathematica, Adobe Illustrator, Corel Draw, MATLAB, etc. - allow the user to save files in one of these formats. Make sure that what you are saving is vector graphics and not a bitmap. If you need help, please write to graphics@msp.org with as many details as you can about how your graphics were generated.

Bundle your figure files into a single archive (using zip, tar, rar or other format of your choice) and upload on the link you been provided at acceptance time. Each figure should be captioned and numbered so that it can float. Small figures occupying no more than three lines of vertical space can be kept in the text ("the curve looks like this:"). It is acceptable to submit a manuscript with all figures at the end, if their placement is specified in the text by means of comments such as "Place Figure 1 here". The same considerations apply to tables.

White Space. Forced line breaks or page breaks should not be inserted in the document. There is no point in your trying to optimize line and page breaks in the original manuscript. The manuscript will be reformatted to use the journal's preferred fonts and layout.

Proofs. Page proofs will be made available to authors (or to the designated corresponding author) at a Web site in PDF format. Failure to acknowledge the receipt of proofs or to return corrections within the requested deadline may cause publication to be postponed. 
Mathematics and Mechanics of Complex Systems vol. 7 no. 3

Effective computation of $\mathrm{SO}(3)$ and $\mathrm{O}(3)$ linear representation symmetry classes

Marc Olive

Low-temperature ratchet current

Justine Louis

Eshelby's inclusion theory in light of Noether's theorem

Salvatore Federico, Mawafag F. Alhasadi and Alfio

Grillo

MEMOCS is a journal of the International Research Center for the Mathematics and Mechanics of Complex Systems at the Università dell' Aquila, Italy.

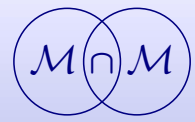

